TRANSACTIONS OF THE

AMERICAN MATHEMATICAL SOCIETY

Volume 361, Number 9, September 2009, Pages 4807-4842

S 0002-9947(09)04828-4

Article electronically published on April 21, 2009

\title{
MULTILOOP REALIZATION OF EXTENDED AFFINE LIE ALGEBRAS AND LIE TORI
}

\author{
BRUCE ALLISON, STEPHEN BERMAN, JOHN FAULKNER, AND ARTURO PIANZOLA
}

\begin{abstract}
An important theorem in the theory of infinite dimensional Lie algebras states that any affine Kac-Moody algebra can be realized (that is to say constructed explicitly) using loop algebras. In this paper, we consider the corresponding problem for a class of Lie algebras called extended affine Lie algebras (EALAs) that generalize affine algebras. EALAs occur in families that are constructed from centreless Lie tori, so the realization problem for EALAs reduces to the realization problem for centreless Lie tori. We show that all but one family of centreless Lie tori can be realized using multiloop algebras (in place of loop algebras). We also obtain necessary and sufficient conditions for two centreless Lie tori realized in this way to be isotopic, a relation that corresponds to isomorphism of the corresponding families of EALAs.
\end{abstract}

An extended affine Lie algebra (EALA) over a field of characteristic zero consists of a Lie algebra $\mathcal{E}$, together with a nondegenerate invariant symmetric bilinear form $($,$) on \mathcal{E}$, and a nonzero finite dimensional ad-diagonalizable subalgebra $\mathcal{H}$ of $\mathcal{E}$, such that a list of natural axioms is imposed (see [N2 and the references therein). (Although, by definition, an EALA consists of a triple $(\mathcal{E}, \mathcal{H},()$,$) , we$ usually abbreviate it as $\mathcal{E}$.) One of the axioms states that the group generated by the isotropic roots of $\mathcal{E}$ is a free abelian group $\Lambda$ of finite rank, and the rank of $\Lambda$ is called the nullity of $\mathcal{E}$. As the term EALA suggests, the defining axioms for an EALA are modeled after the properties of affine Kac-Moody Lie algebras; in fact, affine Kac-Moody Lie algebras are precisely the extended affine Lie algebras of nullity 1 . So it is natural to look for a realization theorem for EALAs of arbitrary nullity $\geq 1$. (Nullity 0 EALAs are finite dimensional simple Lie algebras and we do not consider them in this context.)

The classical procedure for realizing affine Lie algebras using loop algebras proceeds in two steps [K, Chaps. 7 and 8]. In the first step, the derived algebra modulo its centre of the affine algebra is constructed as the loop algebra of a diagram automorphism of a finite dimensional simple Lie algebra. This loop algebra is naturally graded by $Q \times \mathbb{Z}$, where $Q$ is the root lattice of a finite irreducible (but not necessarily reduced) root system. In the second step, the affine algebra itself, together with a Cartan subalgebra and a nondegenerate invariant bilinear form for the affine alge-

Received by the editors September 7, 2007.

2000 Mathematics Subject Classification. Primary 17B65; Secondary 17B67, 17B70.

The first and fourth authors gratefully acknowledge the support of the Natural Sciences and Engineering Research Council of Canada.

(C)2009 American Mathematical Society Reverts to public domain 28 years from publication 
bra, is built from the graded loop algebra by forming a central extension (with a one dimensional centre) and adding a (one dimensional) graded algebra of derivations.

The replacement for the derived algebra modulo its centre in EALA theory is the centreless core of the EALA, and centreless cores of EALAs have been characterized axiomatically as centreless Lie tori [Y2, N1]. A Lie $\Lambda$-torus, or a Lie torus for short, is a $Q \times \Lambda$-graded Lie algebra $\mathcal{L}$ satisfying a simple list of axioms, where $Q$ is the root lattice of an irreducible finite root system $\Delta$ and $\Lambda$ is a free abelian group of finite rank (see subsection 1.1). The type of the root system $\Delta$ is then called the type of $\mathcal{L}$ and the rank of $\Lambda$ is called the nullity of $\mathcal{L}$. A centreless Lie torus is a Lie torus with trivial centre.

Starting with a centreless Lie torus, E. Neher has shown how to carry out the second step of the classical realization procedure [N2]. Specifically, given a centreless Lie torus $\mathcal{L}$ of nullity $n$, Neher gave a simple construction of a family $\{E(\mathcal{L}, D, \tau)\}_{(D, \tau)}$ of EALAs of nullity $n$ parameterized by pairs $(D, \tau)$, where $D$ is a graded Lie algebra of derivations of $\mathcal{L}$ (subject to some additional restrictions) and $\tau$ is a graded invariant 2-cocycle with values in the graded dual $C$ of $D$. (As a vector space, $E(\mathcal{L}, D, \tau)=\mathcal{L} \oplus C \oplus D$, and $\tau$ is used in the description of the multiplication of two elements of $D$. For the largest possible choice of $D$, the algebra of skew-centroidal derivations of $\mathcal{L}, \mathcal{L} \oplus C$ is the universal central extension of $\mathcal{L}$.) Moreover he announced that every EALA $\mathcal{E}$ is isomorphic as an EALA to $E(\mathcal{L}, D, \tau)$ for some $\mathcal{L}$ and $(D, \tau)$ [N2, Thm. 4.2]. (Two EALAs are isomorphic if there is a Lie algebra isomorphism from one to the other preserving the given forms up to nonzero scalar and preserving the given ad-diagonalizable subalgebras.)

Thus, only the first step in the realization procedure needs to be considered - the realization problem for centreless Lie tori. If $k$ is algebraically closed and $n \geq 1$, we describe a construction of a centreless Lie $\mathbb{Z}^{n}$-torus $\operatorname{LT}(\mathfrak{s}, \boldsymbol{\sigma}, \mathfrak{h})$, called a multiloop Lie $\mathbb{Z}^{n}$-torus, starting from a finite dimensional simple Lie algebra $\mathfrak{s}$, a sequence $\boldsymbol{\sigma}$ of $n$-commuting finite order automorphisms satisfying three conditions labeled as (A1)-(A3), and a Cartan subalgebra $\mathfrak{h}$ of the fixed point algebra $\mathfrak{s}^{\sigma}$ of $\boldsymbol{\sigma}$ is $\mathfrak{s}$. In our first main theorem, which we call the realization theorem (see Theorem 3.3.1), we prove that a centreless Lie torus $\mathcal{L}$ of nullity $\geq 1$ is bi-isomorphic (defined below) to a multiloop Lie torus if and only if $\mathcal{L}$ is fgc (that is, $\mathcal{L}$ is finitely generated as a module over its centroid).

We note that when $n=1$ the conditions (A1)-(A3) on a single automorphism $\sigma$ are equivalent to the assumption that $\sigma$ is a diagram automorphism (see subsection 3.4), thus relating our realization theorem to the classical realization theorem for affine algebras.

Our realization theorem is a satisfying answer to the realization problem for centreless Lie tori, and hence for EALAs, since it is known from the work of Neher [N1] that there is only one family of centreless Lie tori that are not fgc, namely the special linear Lie algebras coordinatized by quantum tori whose unit group has infinite commutator group (see Remark 1.4.3). Of course by the classical realization theorem (or directly), we know that these exceptions do not exist in nullity 1 .

We also note that the realization theorem solves a problem raised by J. van de Leur in $\mathrm{vdL}$. In that paper, the author showed using a case-by-case argument that the centreless core of any bi-affine Lie algebras can be constructed as a multiloop algebra. (Bi-affine Lie algebras are extended affine Lie algebras of nullity 2 whose centreless cores had been previously constructed as loop algebras of affine 
algebras.) He pointed out that this was an experimental fact that was not explained by a general theory. Since centreless cores of bi-affine Lie algebras are fgc $\mathrm{ABP}$, Thm. 5.5(iii)], our theorem provides such an explanation.

To complete our understanding of the realization of EALAs, it is important to know when two multiloop Lie tori give rise to the same family of EALAs. To this end, we define an isotope of a Lie $\Lambda$-torus $\mathcal{L}$ as a Lie $\Lambda$-torus obtained from $\mathcal{L}$ by shifting the $\Lambda$-grading by a group homomorphism in $\operatorname{Hom}(Q, \Lambda)$. Two Lie tori $\mathcal{L}$ and $\mathcal{L}^{\prime}$, graded by $Q \times \Lambda$ and $Q^{\prime} \times \Lambda^{\prime}$, respectively, are said to be bi-isomorphic if there is a Lie algebra isomorphism from $\mathcal{L}$ to $\mathcal{L}^{\prime}$ that preserves the gradings up to an isomorphism of $Q$ with $Q^{\prime}$ and an isomorphism of $\Lambda$ with $\Lambda^{\prime}$. Further, they are said to be isotopic if one is bi-isomorphic to an isotope of the other. It turns out that isotopy is exactly what is needed to distinguish between families of EALAs. Indeed, it is shown in [AF, Thm. 6.2] that if two centreless Lie tori $\mathcal{L}$ and $\mathcal{L}^{\prime}$ are isotopic, then the corresponding families of EALAs are isomorphic, in the sense that there is a bijection from the parameter set for the first family onto the parameter set for the second family such that corresponding EALAs are isomorphic. Conversely, if some member of the first family is isomorphic as an EALA to some member of the second family, then $\mathcal{L}$ is isotopic to $\mathcal{L}^{\prime} \mathrm{AF}$, Thm. 6.1]. Hence families of EALAs up to isomorphism are in one-to-one correspondence with isotopy classes of centreless Lie tori.

The next two theorems of the paper, Theorems 4.1.1 and 4.2.5, give necessary and sufficient conditions for two multiloop Lie $\mathbb{Z}^{n}$-tori $\operatorname{LT}(\mathfrak{s}, \boldsymbol{\sigma}, \mathfrak{h})$ and $\operatorname{LT}\left(\mathfrak{s}^{\prime}, \boldsymbol{\sigma}^{\prime}, \mathfrak{h}^{\prime}\right)$ to be bi-isomorphic and isotopic respectively. In view of the above discussion, the second theorem (which is proved using the first theorem) provides necessary and sufficient conditions for the families of EALAs corresponding to $\operatorname{LT}(\mathfrak{s}, \boldsymbol{\sigma}, \mathfrak{h})$ and $\operatorname{LT}\left(\mathfrak{s}^{\prime}, \boldsymbol{\sigma}^{\prime}, \mathfrak{h}^{\prime}\right)$ to be isomorphic. The conditions in both theorems are conditions on the input data $(\mathfrak{s}, \boldsymbol{\sigma}, \mathfrak{h})$ and $\left(\mathfrak{s}^{\prime}, \boldsymbol{\sigma}^{\prime}, \mathfrak{h}^{\prime}\right)$. In particular, the conditions for bi-isomorphism are the existence of an isomorphism $\varphi: \mathfrak{s} \rightarrow \mathfrak{s}^{\prime}$ and a matrix $P \in \mathrm{GL}_{n}(\mathbb{Z})$ so that $\boldsymbol{\sigma}^{\prime}=\varphi \boldsymbol{\sigma}^{P} \varphi^{-1}$, where $(\boldsymbol{\sigma}, P) \rightarrow \boldsymbol{\sigma}^{P}$ is the natural right action of $\mathrm{GL}_{n}(\mathbb{Z})$ on the set of length $n$ sequences of commuting finite order automorphisms of $\mathfrak{s}$.

The primary tools in the proof of the theorems just described are the general results in ABFP on realizations of graded-simple algebras.

In the last section of the paper, we consider the classification problems, up to biisomorphism and up to isotopy, for fgc centreless Lie tori of nullity $n \geq 1$. (As we've mentioned, the last of these problems is equivalent to the classification of families of EALAs of nullity $n$ up to isomorphism.) These problems can be approached in two ways. The first approach uses the coordinatization theorems which have been proved for centreless Lie tori of each type $\Delta$. (These theorems are the work of many authors. See $\mathrm{AF}]$ for a survey of the literature and a sample of these results.) Both classification problems have been solved for some types in this way $\mathrm{AF}$, but other types remain to be considered.

In the last section of this article, we propose a second approach, using multiloop Lie tori and our results, to the classification problems. In this approach we organize the fgc centreless Lie tori by absolute type (see subsection (5.2) rather than by type. Carrying the approach through for a given absolute type $\mathrm{X}_{\ell}$ requires precise information about sequences of commuting finite order automorphisms (or equivalently gradings by a finite abelian group) of a simple Lie algebra of type $\mathrm{X}_{\ell}$ over $k$. The approach to the classification up to bi-isomorphism is summarized in Theorem 
5.3.2. Once the classification up to bi-isomorphism is complete for a given absolute type, one can, at least in principle, apply Theorem 4.2.5 to determine which of the representatives of the bi-isomorphism classes are isotopic. However, it would be interesting to have a more precise method to accomplish this last step.

As an example we solve both classification problems for absolute type $\mathrm{F}_{4}$ using the work of Draper and Martin $[\mathrm{DM}]$ on gradings of the simple Lie algebra of type $\mathrm{F}_{4}$ (see Examples 5.3.4 and 5.3.5). We expect that this approach will also work for (at least some) other types, using classical and recent results about sequences of commuting automorphisms or gradings (see for example [BFM, BSZ, GP, KS]).

To close this introduction, we briefly outline the contents of the paper. In section 1, we recall or prove the properties that we will need about Lie tori. In section 2. we introduce bi-isomorphism and isotopy for Lie tori. Section 3 contains the construction of multiloop Lie tori and the statement and proof of the realization theorem. In section 4 we prove our theorems on bi-isomorphism and isotopy of multiloop Lie tori. Finally, in section 5. we describe our approach to the classification problems.

Assumptions and notation. Throughout this work we assume that $k$ is a field of characteristic 0 . All algebras are assumed to be algebras over $k$. We also assume that $\Lambda$ is an abelian group, written additively. (Beginning in section 3 we will make additional assumptions on $k$ and $\Lambda$, since we will be dealing specifically with multiloop algebras at that point.)

If $\mathcal{B}=\bigoplus_{\lambda \in \Lambda}$ is a $\Lambda$-graded algebra we let $\operatorname{supp}_{\Lambda}(\mathcal{B})=\left\{\lambda \in \Lambda \mid \mathcal{B}^{\lambda} \neq 0\right\}$ denote the $\Lambda$-support of $\mathcal{B}$. If $\mathcal{B}$ is a $\Lambda$-graded algebra and $\mathcal{B}^{\prime}$ is a $\Lambda^{\prime}$-graded algebra, we say that $\mathcal{B}$ and $\mathcal{B}^{\prime}$ are isograded-isomorphic if there exists an algebra isomorphism $\varphi: \mathcal{B} \rightarrow \mathcal{B}^{\prime}$ and a group isomorphism $\varphi_{\mathrm{gr}}: \Lambda \rightarrow \Lambda^{\prime}$ such that $\varphi\left(\mathcal{B}^{\lambda}\right)=\mathcal{B}^{\prime} \varphi_{\mathrm{gr}}(\lambda)$ for $\lambda \in \Lambda$. If $\left\langle\operatorname{supp}_{\Lambda}(\mathcal{B})\right\rangle=\Lambda$, then $\varphi_{\mathrm{gr}}$ is uniquely determined by $\varphi$.

If $\mathcal{L}$ is a Lie algebra, we denote the centre of $\mathcal{L}$ by $Z(\mathcal{L})$, and we call $\mathcal{L}$ centreless if $Z(\mathcal{L})=0$.

If $S$ is a subset of a group, we denote the subgroup generated by $S$ as $\langle S\rangle$.

\section{LIE TORI}

1.1. Definitions and basic properties. We begin by recalling the definition of a Lie torus and describing some of its properties. In order to discuss Lie tori we first establish some conventions and notation for root systems.

Convention 1.1.1. As in AABGP, AG and N1 it will be convenient for us to work with root systems that contain 0 . So by a finite irreducible root system we will mean a finite subset $\Delta$ of a finite dimensional vector space $X$ over $k$ such that $0 \in \Delta$ and $\Delta^{\times}:=\Delta \backslash\{0\}$ is a finite irreducible root system in $X$ in the usual sense (see [B1, Chap. VI, §1, Définition 1]).

Assume for the rest of this paper (unless indicated to the contrary) that $\Delta$ is a finite irreducible root system in a finite dimensional vector space $\mathcal{X}$ over $k$. Thus, $\Delta$ has one of the following types:

$$
\mathrm{A}_{\ell}(\ell \geq 1), \mathrm{B}_{\ell}(\ell \geq 2), \mathrm{C}_{\ell}(\ell \geq 3), \mathrm{D}_{\ell}(\ell \geq 4), \mathrm{E}_{6}, \mathrm{E}_{7}, \mathrm{E}_{8}, \mathrm{~F}_{4}, \mathrm{G}_{2}, \mathrm{BC}_{\ell}(\ell \geq 1)
$$

[B1, Chap. VI, §4]. It will sometimes be convenient to say that $\Delta$ has type $\mathrm{B}_{\ell}$ with

$\ell \geq 1$, which means that $\Delta$ has type $\mathrm{A}_{1}$ or $\mathrm{B}_{\ell}(\ell \geq 2)$. Recall that $\Delta$ is said to be 
reduced if $2 \alpha \notin \Delta^{\times}$for $\alpha \in \Delta^{\times}$. Then, $\Delta$ is not reduced if and only if $\Delta$ is of type $\mathrm{BC}_{\ell}$, where $\ell \geq 1$.

Notation 1.1.2. We will use the following notation for the root system $\Delta$. Let

$$
Q=Q(\Delta):=\operatorname{span}_{\mathbb{Z}}(\Delta)
$$

be the root lattice of $\Delta$. Let $X^{*}$ denote the dual space of $X$, and let $\langle\rangle:, X \times X^{*} \rightarrow k$ denote the natural pairing of $\mathcal{X}$ with $X^{*}$. If $\alpha \in \Delta^{\times}, \alpha^{\vee}$ will denote the coroot of $\alpha$ in $X^{*}$; that is, $\alpha^{\vee}$ is the unique element of $X^{*}$ such that $\left\langle\alpha, \alpha^{\vee}\right\rangle=2$ and the map $\beta \rightarrow \beta-\left\langle\beta, \alpha^{\vee}\right\rangle \alpha$ stabilizes $\Delta$. Let

$$
\Delta_{\mathrm{sh}}=\text { set of short roots in } \Delta
$$

that is, $\Delta_{\text {sh }}$ is the set of nonzero roots of minimum length in $\Delta$. Let

$$
\Delta_{\text {ind }}^{\times}:=\left\{\alpha \in \Delta^{\times} \mid \frac{1}{2} \alpha \notin \Delta\right\}
$$

denote the set of indivisible roots in $\Delta$, and let

$$
\Delta_{\text {ind }}:=\Delta_{\text {ind }}^{\times} \cup\{0\} .
$$

Going in the reverse direction, we can enlarge $\Delta$ by setting

$$
\Delta_{\mathrm{en}}= \begin{cases}\Delta \cup 2 \Delta_{\mathrm{sh}}, & \text { if } \Delta \text { has type } \mathrm{B}_{\ell}, \ell \geq 1 ; \\ \Delta, & \text { otherwise. }\end{cases}
$$

Note that both $\Delta_{\text {ind }}$ and $\Delta_{\text {en }}$ are irreducible root systems in $\mathcal{X}$, and $\Delta_{\text {ind }}$ is reduced.

Remark 1.1.3. If $\Delta$ is a finite irreducible root system in $X$ and $\alpha \rightarrow \tilde{\alpha}$ is a $k$ linear isomorphism of $\mathcal{X}$ onto a vector space $\tilde{X}$, then $\widetilde{\Delta}:=\{\tilde{\alpha} \mid \alpha \in \Delta\}$ is a finite irreducible root system in $\tilde{x}$ and we have

$$
\left\langle\alpha, \beta^{\vee}\right\rangle=\left\langle\tilde{\alpha}, \tilde{\beta}^{\vee}\right\rangle
$$

for $\alpha \in \Delta$ and $\beta \in \Delta^{\times}$, where $\tilde{\beta}^{\vee}$ is the coroot of $\tilde{\beta}$ in $\tilde{X}^{*}$.

Example 1.1.4. Suppose that $\mathfrak{h}$ is a finite dimensional ad-diagonalizable subalgebra of a Lie algebra $\mathfrak{g}$. Let

$$
\mathfrak{g}_{\alpha}=\{x \in \mathfrak{g} \mid[h, x]=\alpha(h) x \text { for } h \in \mathfrak{h}\} .
$$

Then, we have the decomposition $\mathfrak{g}=\bigoplus_{\alpha \in \mathfrak{h}^{*}} \mathfrak{g}_{\alpha}$, called the root space decomposition of $\mathfrak{g}$ relative to the adjoint action of $\mathfrak{h}$. We set

$$
\Delta(\mathfrak{g}, \mathfrak{h})=\left\{\alpha \in \mathfrak{h}^{*} \mid \mathfrak{g}_{\alpha} \neq 0\right\} .
$$

In many cases, although not in general, $\Delta(\mathfrak{g}, \mathfrak{h})$ is a finite irreducible root system in $\mathfrak{h}^{*}$. In particular, if $\mathfrak{g}$ is finite dimensional split simple and $\mathfrak{h}$ is a splitting Cartan subalgebra of $\mathfrak{g}$, then $\Delta(\mathfrak{g}, \mathfrak{h})$ is a reduced finite irreducible root system in $\mathfrak{h}^{*}$ [J, §IV.1], [B2, Chap. VIII, §2, Th. 2].

Lie tori are Lie algebras that are graded by the direct product $Q \times \Lambda$ and satisfy some additional axioms that we are going to recall. To do this we first introduce some notation for $Q \times \Lambda$-graded algebras.

Notation 1.1.5. Let

$$
\mathcal{L}=\bigoplus_{(\alpha, \lambda) \in Q \times \Lambda} \mathcal{L}_{\alpha}^{\lambda}
$$


be a $Q \times \Lambda$-graded algebra. (It is convenient as in $\mathrm{AG}$, Y2 and [N1 to use the notation $\mathcal{L}_{\alpha}^{\lambda}$ rather than $\mathcal{L}^{(\alpha, \lambda)}$ or $\mathcal{L}_{(\alpha, \lambda)}$ for the space of elements of degree $(\alpha, \lambda)$ in $\mathcal{L}$.) Then $\mathcal{L}=\bigoplus_{\lambda \in \Lambda} \mathcal{L}^{\lambda}$ is $\Lambda$-graded and $\mathcal{L}=\bigoplus_{\alpha \in Q} \mathcal{L}_{\alpha}$ is $Q$-graded, with

$$
\mathcal{L}^{\lambda}=\bigoplus_{\alpha \in Q} \mathcal{L}_{\alpha}^{\lambda} \text { for } \lambda \in \Lambda \quad \text { and } \quad \mathcal{L}_{\alpha}=\bigoplus_{\lambda \in \Lambda} \mathcal{L}_{\alpha}^{\lambda} \text { for } \alpha \in Q,
$$

and we have

$$
\mathcal{L}_{\alpha}^{\lambda}=\mathcal{L}_{\alpha} \cap \mathcal{L}^{\lambda} .
$$

Conversely if $\mathcal{L}$ has a $Q$-grading and a $\Lambda$-grading that are compatible (which means that each $\mathcal{L}_{\alpha}$ is a $\Lambda$-graded subspace of $\mathcal{L}$ or equivalently that each $\mathcal{L}^{\lambda}$ is a $Q$-graded subspace of $\mathcal{L}$ ), then $\mathcal{L}$ is $Q \times \Lambda$-graded with $\mathcal{L}_{\alpha}^{\lambda}=\mathcal{L}_{\alpha} \cap \mathcal{L}^{\lambda}$. Hence, a $Q \times \Lambda$-graded algebra $\mathcal{L}$ has three different associated support sets, namely the $Q \times \Lambda$-support, the $Q$-support and the $\Lambda$-support denoted respectively by $\operatorname{supp}_{Q \times \Lambda}(\mathcal{L}), \operatorname{supp}_{Q}(\mathcal{L})$ and $\operatorname{supp}_{\Lambda}(\mathcal{L})$.

We now recall the definition of a Lie torus. Lie tori were introduced by Y. Yoshii [Y2, Y3] and further studied by E. Neher in [N1, [N2]. (See Remark 1.2.3 below.)

Definition 1.1.6. A Lie $\Lambda$-torus of type $\Delta$ is a $Q \times \Lambda$-graded Lie algebra $\mathcal{L}$ over $k$ which (with the notation of (1.1.5) ) satisfies:

$(\mathrm{LT} 1) \operatorname{supp}_{Q}(\mathcal{L}) \subseteq \Delta$.

(LT2) (i) $\left(\Delta_{\text {ind }}^{\times}, 0\right) \subseteq \operatorname{supp}_{Q \times \Lambda}(\mathcal{L})$.

(ii) If $(\alpha, \lambda) \in \operatorname{supp}_{Q \times \Lambda}(\mathcal{L})$ and $\alpha \neq 0$, then there exist elements $e_{\alpha}^{\lambda} \in \mathcal{L}_{\alpha}^{\lambda}$ and $f_{\alpha}^{\lambda} \in \mathcal{L}_{-\alpha}^{-\lambda}$ such that

$$
\mathcal{L}_{\alpha}^{\lambda}=k e_{\alpha}^{\lambda}, \quad \mathcal{L}_{-\alpha}^{-\lambda}=k f_{\alpha}^{\lambda},
$$

and

$$
\left[\left[e_{\alpha}^{\lambda}, f_{\alpha}^{\lambda}\right], x_{\beta}\right]=\left\langle\beta, \alpha^{\vee}\right\rangle x_{\beta}
$$

for $x_{\beta} \in \mathcal{L}_{\beta}, \beta \in Q$.

(LT3) $\mathcal{L}$ is generated as an algebra by the spaces $\mathcal{L}_{\alpha}, \alpha \in \Delta^{\times}$.

$(\mathrm{LT} 4)\left\langle\operatorname{supp}_{\Lambda}(\mathcal{L})\right\rangle=\Lambda$.

In that case we call $Q$ the root-grading group and we call the $Q$-grading of $\mathcal{L}$ the root grading. Similarly, we call $\Lambda$ the external grading group and we call the $\Lambda$-grading of $\mathcal{L}$ the external grading. If $\Delta$ has type $\mathrm{X}_{\ell}$, with $\mathrm{X}_{\ell}$ as in (11), we also say that $\mathcal{L}$ has type $X_{\ell}$. Finally if $\Lambda$ is free abelian of rank $n \geq 0$, we say that $\mathcal{L}$ has nullity $n$.

Remark 1.1.7. Suppose that $\mathcal{L}$ is a Lie $\Lambda$-torus of type $\Delta$. Unless indicated otherwise, we will assume that we have made a fixed choice of elements $e_{\alpha}^{\lambda}$ and $f_{\alpha}^{\lambda}$ as in (LT2)(ii) for $(\alpha, \lambda) \in \operatorname{supp}_{Q \times \Lambda}(\mathcal{L})$ with $\alpha \in \Delta^{\times}$. Note that both the elements $e_{\alpha}^{\lambda}$ and $f_{\alpha}^{\lambda}$ are nonzero, since otherwise we would have $\left\langle\beta, \alpha^{\vee}\right\rangle=0$ for all $\beta \in \operatorname{supp}_{Q}(\mathcal{L})$ and hence for all $\beta \in \mathcal{X}$. Consequently the triple $\left(e_{\alpha}^{\lambda},\left[e_{\alpha}^{\lambda}, f_{\alpha}^{\lambda}\right], f_{\alpha}^{\lambda}\right)$ forms an sl ${ }_{2}$-triple in $\mathcal{L}$.

Remark 1.1.8. If $\mathcal{L}$ is a $Q \times \Lambda$-graded algebra, then the subalgebra of $\mathcal{L}$ generated by the spaces $\bigcup_{\alpha \in \Delta \times} \mathcal{L}_{\alpha}$ is a $Q \times \Lambda$-graded ideal of $\mathcal{L}$. Moreover, if $\mathcal{L}$ satisfies (LT2)(i), this ideal is not zero. Thus, if $\mathcal{L}$ is a graded-simple $Q \times \Lambda$-graded algebra satisfying (LT2)(i), then (LT3) automatically holds. 
Remark 1.1.9. If $\mathcal{L}$ is a Lie $\Lambda$-torus of type $\Delta,(\alpha, \lambda) \in \operatorname{supp}_{Q \times \Lambda}(\mathcal{L})$ and $\alpha \neq 0$, we define $\theta_{\alpha}^{\lambda} \in \operatorname{Aut}_{k}(\mathcal{L})$ by

$$
\theta_{\alpha}^{\lambda}=\exp \left(\operatorname{ad}\left(e_{\alpha}^{\lambda}\right)\right) \exp \left(\operatorname{ad}\left(-f_{\alpha}^{\lambda}\right)\right) \exp \left(\operatorname{ad}\left(e_{\alpha}^{\lambda}\right)\right) .
$$

Then, as noted in [Y3, §5] (see the argument in [AABGP, Prop. 1.27]), we have

$$
\theta_{\alpha}^{\lambda}\left(\mathcal{L}_{\beta}^{\mu}\right)=\mathcal{L}_{w_{\alpha}(\beta)}^{\mu-\left\langle\beta, \alpha^{\vee}\right\rangle \lambda}
$$

for $(\beta, \mu) \in Q \times \Lambda$, where $w_{\alpha}$ is the Weyl reflection corresponding to $\alpha \in \Delta$.

We next examine the $Q$-support of a Lie torus.

Lemma 1.1.10. Let $\mathcal{L}$ be a Lie $\Lambda$-torus of type $\Delta$. Then

$$
\operatorname{supp}_{Q}(\mathcal{L})= \begin{cases}\Delta & \text { if } \Delta \text { is reduced; } \\ \Delta \text { or } \Delta_{\text {ind }} & \text { if } \Delta \text { is not reduced } .\end{cases}
$$

Consequently, $\operatorname{supp}_{Q}(\mathcal{L})$ is a finite irreducible root system in $\mathcal{X}$ and

$$
\Delta_{\text {ind }}=\operatorname{supp}_{Q}(\mathcal{L})_{\text {ind }} .
$$

Proof. By $(\mathrm{LT} 1)$ and $(\mathrm{LT} 2)(\mathrm{i})$, we have $\Delta_{\text {ind }}^{\times} \subseteq \operatorname{supp}_{Q}(\mathcal{L}) \subseteq \Delta$. Moreover $0 \in$ $\operatorname{supp}_{Q}(\mathcal{L})$ since $0 \neq\left[e_{\alpha}^{0}, f_{\alpha}^{0}\right] \in \mathcal{L}_{0}$ for $\alpha \in \Delta_{\text {ind }}^{\times}$. Thus

$$
\Delta_{\text {ind }} \subseteq \operatorname{supp}_{Q}(\mathcal{L}) \subseteq \Delta .
$$

If $\Delta$ is reduced, then $\Delta=\Delta_{\text {ind }}$, which implies our conclusion. Suppose that $\Delta$ is not reduced. Now $\operatorname{supp}_{Q}(\mathcal{L})$ is closed under the action of the Weyl group of $\Delta$ by (4). This fact together with (5) implies our conclusion.

Remark 1.1.11. Suppose that $\mathcal{L}$ is a Lie $\Lambda$-torus of type $\Delta$. It is sometimes convenient to assume the following additional axiom:

$\left(\right.$ LT5) $\operatorname{supp}_{Q}(\mathcal{L})=\Delta$.

Note that if $\mathcal{L}$ does not satisfy (LT5), then by Lemma 1.1 .10 it follows that $\Delta$ is not reduced and $\operatorname{supp}_{Q}(\mathcal{L})=\Delta_{\text {ind }}$. In that case $\mathcal{L}$ is also a Lie $\Lambda$-torus of type $\Delta_{\text {ind }}$ and as such it satisfies (LT5). Thus there is really no loss of generality in assuming (LT5) when convenient.

If $\mathcal{L}$ is a Lie $\Lambda$-torus of type $\Delta$, we define

$$
\Lambda_{\alpha}=\left\{\lambda \in \Lambda \mid \mathcal{L}_{\alpha}^{\lambda} \neq 0\right\}
$$

for $\alpha \in Q$. Thus,

$$
\operatorname{supp}_{\Lambda}(\mathcal{L})=\bigcup_{\alpha \in Q} \Lambda_{\alpha},
$$

and $\Lambda_{\alpha} \neq \emptyset$ if and only if $\alpha \in \operatorname{supp}_{Q}(\mathcal{L})$. Note that if $\alpha, \beta \in \operatorname{supp}_{Q}(\mathcal{L})$ and $\alpha \neq 0$, we have, by (4),

$$
\Lambda_{\beta}-\left\langle\beta, \alpha^{\vee}\right\rangle \Lambda_{\alpha} \subseteq \Lambda_{w_{\alpha}(\beta)} .
$$

Using [Y3], we have the following properties of the sets $\Lambda_{\alpha}$. (See also AABGP, Chapter II].)

Lemma 1.1.12. Suppose that $\mathcal{L}$ is a Lie $\Lambda$-torus of type $\Delta$.

(i) If $\alpha \in \Delta_{\text {ind }}^{\times}$, then $0 \in \Lambda_{\alpha}$.

(ii) If $\alpha \in \operatorname{supp}_{Q}(\mathcal{L})^{\times}$, then $\Lambda_{\alpha}+2 \Lambda_{\alpha} \subseteq \Lambda_{\alpha}$ and $-\Lambda_{\alpha}=\Lambda_{\alpha}$.

(iii) If $\alpha, \beta \in \operatorname{supp}_{Q}(\mathcal{L})^{\times}$have the same length, then $\Lambda_{\alpha}=\Lambda_{\beta}$.

(iv) If $\alpha \in \operatorname{supp}_{Q}(\mathcal{L})^{\times}$and $\beta \in \Delta_{\mathrm{sh}}$, then $\Lambda_{\alpha} \subseteq \Lambda_{\beta}$. 
(v) If $\beta \in \Delta_{\mathrm{sh}}$, then $\operatorname{supp}_{\Lambda}(\mathcal{L})=\Lambda_{0}=\Lambda_{\beta}+\Lambda_{\beta}, \Lambda=\left\langle\Lambda_{\beta}\right\rangle, \Lambda_{\beta}+2 \Lambda \subseteq \Lambda_{\beta}$ and $2 \Lambda \subseteq \Lambda_{\beta}$

(vi) $2 \Lambda \subseteq \operatorname{supp}_{Q}(\mathcal{L})$

Proof. (i) follows from (LT2)(i). Because (7) holds, we may invoke the results of Y3, §3], which give (ii), (iii) and (iv). For (v), the equality $\Lambda_{0}=\Lambda_{\beta}+\Lambda_{\beta}$ is proved in [Y3, Thm. 5.1]. Then, since $\operatorname{supp}_{\Lambda}(\mathcal{L})=\bigcup_{\alpha \in Q} \Lambda_{\alpha}$, we see using (iv) and (i) that $\operatorname{supp}_{\Lambda}(\mathcal{L})=\Lambda_{0}$. Hence, since $\Lambda=\left\langle\operatorname{supp}_{\Lambda}(\mathcal{L})\right\rangle$, we have $\Lambda=\left\langle\Lambda_{\beta}\right\rangle$. Thus, by (ii) (applied to $\alpha=\beta$ ), we have $\Lambda_{\beta}+2 \Lambda \subseteq \Lambda_{\beta}$. Since $0 \in \Lambda_{\beta}$, this gives $2 \Lambda \subseteq \Lambda_{\beta}$. Finally, for (vi), choose $\beta \in \Delta_{\text {sh }}$. Then, $2 \Lambda \subseteq \Lambda_{\beta} \subseteq \operatorname{supp}_{\Lambda}(\mathcal{L})$.

\subsection{The root-grading pair.}

Definition 1.2.1. Let $\mathcal{L}$ be a Lie $\Lambda$-torus of type $\Delta$. Following $\mathbb{N 1}$, we define the root-grading pair for $\mathcal{L}$ to be the pair $(\mathfrak{g}, \mathfrak{h})$ of subalgebras of $\mathcal{L}$ where $\mathfrak{g}$ is the subalgebra of $\mathcal{L}$ generated by $\left\{\mathcal{L}_{\alpha}^{0}\right\}_{\alpha \in \Delta^{\times}}$and $\mathfrak{h}=\sum_{\alpha \in \Delta^{\times}}\left[\mathcal{L}_{\alpha}^{0}, \mathcal{L}_{-\alpha}^{0}\right]$. Notice that $\mathfrak{h}$ is a subalgebra of $\mathfrak{g}, \mathfrak{g} \subseteq \mathcal{L}^{0}$ and $\mathfrak{h} \subseteq \mathcal{L}_{0}^{0}$. We will see below in (9) that the subalgebra $\mathfrak{h}$ completely determines the root grading of $\mathcal{L}$.

The next proposition summarizes the basic properties of the root-grading pair of a Lie torus. Parts (i)-(v) were announced in [N1, §3] in the case when $\Lambda$ is a finitely generated free abelian group. We sketch a proof of the proposition for the convenience of the reader.

Proposition 1.2.2. Let $\mathcal{L}$ be a Lie $\Lambda$-torus of type $\Delta$ with root-grading pair $(\mathfrak{g}, \mathfrak{h})$.

(i) If $\alpha \in \Delta_{\text {ind }}^{\times}$, then $\mathcal{L}_{2 \alpha}^{0}=0$.

(ii) $\mathfrak{g}$ is a finite dimensional split simple Lie algebra with splitting Cartan subalgebra $\mathfrak{h}$.

(iii) There is a unique linear isomorphism $\alpha \rightarrow \tilde{\alpha}$ of $\mathcal{X}$ onto $\mathfrak{h}^{*}$ such that $\widetilde{\Delta_{\text {ind }}}=$ $\Delta(\mathfrak{g}, \mathfrak{h})$ and

$$
\left[e_{\alpha}^{0}, f_{\alpha}^{0}\right]=\tilde{\alpha}^{\vee}
$$

for $\alpha \in \Delta_{\text {ind }}^{\times}$. (See Remark 1.1 .3 for the notation. Here $\tilde{\alpha}^{\vee} \in\left(\mathfrak{h}^{*}\right)^{*}=\mathfrak{h}$.)

(iv) If $\alpha \in Q$, then

$$
\mathcal{L}_{\alpha}=\{x \in \mathcal{L} \mid[h, x]=\tilde{\alpha}(h) x \text { for } h \in \mathfrak{h}\} .
$$

(v) If $(\alpha, \lambda) \in \operatorname{supp}_{Q \times \Lambda}(\mathcal{L})$ and $\alpha \in \Delta^{\times}$, then

$$
\left[e_{\alpha}^{\lambda}, f_{\alpha}^{\lambda}\right]-\tilde{\alpha}^{\vee} \in Z(\mathcal{L}) .
$$

(vi) We have

$$
\begin{aligned}
& \mathcal{L}^{0}=\mathfrak{g} \oplus\left(Z(\mathcal{L}) \cap \mathcal{L}^{0}\right) \quad \text { and } \quad \mathcal{L}_{0}^{0}=\mathfrak{h} \oplus\left(Z(\mathcal{L}) \cap \mathcal{L}^{0}\right), \\
& \text { so } \mathfrak{g}=\left[\mathcal{L}^{0}, \mathcal{L}^{0}\right] \quad \text { and } \mathfrak{h}=\left[\mathcal{L}^{0}, \mathcal{L}^{0}\right] \cap \mathcal{L}_{0} . \\
& \text { Consequently, if } \mathcal{L} \text { is centreless, then } \mathcal{L}^{0}=\mathfrak{g} \text { and } \mathcal{L}_{0}^{0}=\mathfrak{h} .
\end{aligned}
$$

Proof. (i): This is easily proved by contradiction using the representation theory of the $\mathrm{sl}_{2}$-triple $\left(e_{\alpha}^{0},\left[e_{\alpha}^{0}, f_{\alpha}^{0}\right], f_{\alpha}^{0}\right)$ and the fact that $\left[\mathcal{L}_{\alpha}^{0}, \mathcal{L}_{-\alpha}^{0}\right]=k\left[e_{\alpha}^{0}, f_{\alpha}^{0}\right]$. (See, for example, the proof of Theorem 1.29(c) of [AABGP Prop. 6.3].)

(ii)-(v): Uniqueness in (iii) is clear, so we need to prove the other properties in (ii) $-(\mathrm{v})$. Let $\Pi=\left\{\alpha_{1}, \ldots, \alpha_{\ell}\right\}$ be a base for the root system $\Delta_{\text {ind }}$. Let

$$
e_{i}=e_{\alpha_{i}}^{0}, \quad f_{i}=f_{\alpha_{i}}^{0} \quad \text { and } \quad h_{i}=\left[e_{i}, f_{i}\right]
$$


for $1 \leq i \leq \ell$. Let $\tilde{\mathfrak{g}}$ be the subalgebra of $\mathfrak{g}$ generated by the elements $\left\{e_{i}, f_{i}\right\}_{i=1}^{\ell}$, and let $\tilde{\mathfrak{h}}$ be the subspace of $\mathfrak{h}$ spanned by $\left\{h_{i}\right\}_{i=1}^{\ell}$. (We will see below that $\tilde{\mathfrak{g}}=\mathfrak{g}$ and $\tilde{\mathfrak{h}}=\mathfrak{h}$.) It is easy to check using (3) that the elements $\left\{e_{i}, f_{i}, h_{i}\right\}_{i=1}^{\ell}$ satisfy the Chevalley-Serre relations determined by the Cartan matrix $\left(\left\langle\alpha_{i}, \alpha_{j}^{\vee}\right\rangle\right)$. Hence, by [B2, Chap. VIII, §4, Th. 1], $\mathfrak{\mathfrak { g }}$ is a finite dimensional split simple Lie algebra with splitting Cartan subalgebra $\tilde{\mathfrak{h}}$, and there is a linear isomorphism $\alpha \rightarrow \tilde{\alpha}$ such that $\widetilde{\Delta_{\text {ind }}}=\Delta(\tilde{\mathfrak{g}}, \tilde{\mathfrak{h}})$ and

$$
\tilde{\alpha}\left(h_{i}\right)=\left\langle\alpha, \alpha_{i}^{\vee}\right\rangle
$$

for $i=1, \ldots, \ell$.

Now we set $\mathcal{L}_{\tilde{\alpha}}=\{x \in \mathcal{L} \mid[h, x]=\tilde{\alpha}(h) x$ for $h \in \tilde{\mathfrak{h}}\}$ for $\alpha \in Q$. Then using (3) and (11) one checks that $\mathcal{L}_{\alpha} \subseteq \mathcal{L}_{\tilde{\alpha}}$ for $\alpha \in Q$. Hence since $\mathcal{L}=\bigoplus_{\alpha \in Q} \mathcal{L}_{\alpha}$ and since the sum $\bigoplus_{\alpha \in Q} \mathcal{L}_{\tilde{\alpha}}$ is direct we have $\mathcal{L}_{\alpha}=\mathcal{L}_{\tilde{\alpha}}$ for $\alpha \in Q$. So

$$
\mathcal{L}_{\alpha}=\{x \in \mathcal{L} \mid[h, x]=\tilde{\alpha}(h) x \text { for } h \in \tilde{\mathfrak{h}}\}
$$

for $\alpha \in Q$.

Next if $\alpha \in \Delta_{\text {ind }}^{\times}$, then $\tilde{\alpha}$ is a nonzero root of $\tilde{\mathfrak{g}}$ relative to $\tilde{\mathfrak{h}}$, so, by (12), we have $\tilde{\mathfrak{g}} \cap \mathcal{L}_{\alpha} \neq 0$. Hence $\tilde{\mathfrak{g}} \cap \mathcal{L}_{\alpha}^{0} \neq 0$, so, by the 1-dimensionality of $\mathcal{L}_{\alpha}^{0}$, we have $\mathcal{L}_{\alpha}^{0} \subseteq \tilde{\mathfrak{g}}$ for $\alpha \in \Delta_{\text {ind }}^{\times}$. But if $\alpha \in \Delta^{\times} \backslash \Delta_{\text {ind }}^{\times}$we have $\mathcal{L}_{\alpha}^{0}=0$ by (i). Hence $\mathcal{L}_{\alpha}^{0} \subseteq \tilde{\mathfrak{g}}$ for $\alpha \in \Delta^{\times}$, so $\mathfrak{g} \subseteq \tilde{\mathfrak{g}}$. But by definition we have $\tilde{\mathfrak{g}} \subseteq \mathfrak{g}$, so $\tilde{\mathfrak{g}}=\mathfrak{g}$. Consequently, $\tilde{\mathfrak{h}} \subseteq \mathfrak{h} \subseteq \tilde{\mathfrak{g}}$ and, by $(12), \mathfrak{h}$ centralizes $\tilde{\mathfrak{h}}$. So $\tilde{\mathfrak{h}}=\mathfrak{h}$.

It remains to show (8) and (10). Since $\mathfrak{g} \cap Z(\mathcal{L})=0$, it suffices to show (10). For this let $(\alpha, \lambda) \in \operatorname{supp}_{Q \times \Lambda}(\mathcal{L})$ with $\alpha \in \Delta^{\times}$. Then using (2), (3) and (12), one checks that $\left[\left[e_{\alpha}^{\lambda}, f_{\alpha}^{\lambda}\right]-\tilde{\alpha}^{\vee}, x_{\beta}^{\mu}\right]=0$ for $\beta \in \Delta, \mu \in \Lambda$ and $x_{\beta}^{\mu} \in \mathcal{L}_{\beta}^{\mu}$. This proves (10).

(vi): It suffices to prove that $\mathcal{L}^{0}=\mathfrak{g}+Z(\mathcal{L}) \cap \mathcal{L}^{0}$ and $\mathcal{L}_{0}^{0}=\mathfrak{h}+Z(\mathcal{L}) \cap \mathcal{L}^{0}$. In fact it's enough to prove the first of these equations since $\mathcal{L}_{0}^{0}$ is the centralizer in $\mathcal{L}^{0}$ of $\mathfrak{h}$ (by (9)). To do this it's enough to show that $\mathcal{L}_{\alpha}^{0} \subseteq \mathfrak{g}+Z(\mathcal{L})$ for $\alpha \in \Delta$. But this is true by definition of $\mathfrak{g}$ if $\alpha \neq 0$. On the other hand, using (LT3) and (10), we have $\mathcal{L}_{0}^{0}=\sum_{\alpha \in \Delta^{\times}} \sum_{\lambda \in \Lambda}\left[\mathcal{L}_{\alpha}^{\lambda}, \mathcal{L}_{-\alpha}^{-\lambda}\right] \subseteq \mathfrak{h}+Z(\mathcal{L})$.

Remark 1.2.3. As noted already, Lie $\Lambda$-tori were first defined by Yoshii in YY2, Y3, whereas the definition given above in Definition 1.1.6 is due to Neher N1. Yoshii's original definition assumed that $\mathcal{L}$ is a root-graded Lie algebra of type $\Delta$ as defined in [ABG, Chapter 1]. Yoshii and Neher's definitions are equivalent in view of Proposition 1.2.2 (see [N1, §3]).

Remark 1.2.4. If $\Lambda=0$ and $\mathcal{L}$ is a Lie $\Lambda$-torus, then, by Proposition 1.2 .2 (vi), we have $\mathcal{L}=\mathfrak{g} \oplus Z(\mathcal{L})$ and therefore, by (LT4) and (9), we have $\mathcal{L}=\mathfrak{g}$. Thus, $\mathcal{L}$ is a finite dimensional split simple Lie algebra.

1.3. Fgc algebras. A class of Lie tori of particular interest from the point of view of EALA theory is the class of fgc centreless Lie tori. To discuss this class, we first recall some facts about the centroid and the fgc condition for algebras in general (not necessarily Lie algebras). (See [BN, §2.2] and [ABFP, §4] for more details.)

Definition 1.3.1. Suppose that $\mathcal{B}$ is an algebra. The centroid of $\mathcal{B}$ is the subalgebra $C_{k}(\mathcal{B})$ of $\operatorname{End}_{k}(\mathcal{B})$ consisting of the $k$-linear endomorphisms of $\mathcal{B}$ that commute with all left and right multiplication by elements of $\mathcal{B}$. We usually write $C_{k}(\mathcal{B})$ simply as $C(\mathcal{B})$. $\mathcal{B}$ is said to be central-simple if $\mathcal{B}$ is simple and $C(\mathcal{B})=k 1$. 
Recall that a finite dimensional simple algebra is automatically central-simple if $k$ is algebraically closed [J, Theorem 10.1].

Definition 1.3.2. Suppose that $\mathcal{B}=\bigoplus_{\lambda \in \Lambda} \mathcal{B}^{\lambda}$ is a $\Lambda$-graded algebra. $\mathcal{B}$ is said to be graded-simple if $\mathcal{B B} \neq 0$ and the only graded ideals of $\mathcal{B}$ are 0 and $\mathcal{B}$. If $\mathcal{B}$ is graded-simple, then $C(\mathcal{B})=\bigoplus_{\lambda \in \Lambda} C(\mathcal{B})^{\lambda}$ is a unital commutative associative $\Lambda$-graded algebra, where

$$
C(\mathcal{B})^{\lambda}=\left\{c \in C(\mathcal{B}) \mid c \mathcal{B}^{\mu} \subseteq \mathcal{B}^{\lambda+\mu} \text { for } \mu \in \Lambda\right\}
$$

for $\lambda \in \Lambda$ [BN, Proposition 2.16]. Finally $\mathcal{B}$ is said to be graded-central-simple if $\mathcal{B}$ is graded-simple and $C(\mathcal{B})^{0}=k 1$.

Remark 1.3.3. If $\mathcal{B}$ is a graded-simple $\Lambda$-graded algebra and $\operatorname{dim}\left(\mathcal{B}^{\lambda}\right)=1$ for some $\lambda \in \Lambda$, then $\mathcal{B}$ is graded-central-simple [ABFP, Lemma 4.3.4].

Definition 1.3.4. Suppose that $\mathcal{B}=\bigoplus_{\lambda \in \Lambda} \mathcal{B}^{\lambda}$ is a graded-simple $\Lambda$-graded algebra. The support $\Gamma_{\Lambda}(\mathcal{B})=\left\{\gamma \in \Lambda \mid C(\mathcal{B})^{\gamma} \neq 0\right\}$ of $C(\mathcal{B})$ in $\Lambda$ is a subgroup of $\Lambda[\mathrm{BN}$, Proposition 2.16] which we call the central grading group of $\mathcal{B}$. We often write $\Gamma_{\Lambda}(\mathcal{B})$ simply as $\Gamma(\mathcal{B})$.

As noted in ABFP, Lemmas 4.3.5 and 4.3.8], we have the following:

Lemma 1.3.5. Suppose that $\mathcal{B}$ is a graded-central-simple $\Lambda$-graded algebra. Then $C(\mathcal{B})$ has a basis $\left\{c_{\gamma}\right\}_{\gamma \in \Gamma(\mathcal{B})}$ such that $c_{\gamma} \in C(\mathcal{B})^{\gamma}$ is a unit of $C(\mathcal{B})$ for $\gamma \in \Gamma(\mathcal{B})$. Moreover, if $\Lambda$ is finitely generated and free or if $k$ is algebraically closed, then $C(\mathcal{B})$ is isomorphic as a $\Lambda$-graded algebra to the group algebra $k[\Gamma(\mathcal{B})]$.

Definition 1.3.6. If $\mathcal{B}$ is an algebra, then $\mathcal{B}$ is naturally a left $C(\mathcal{B})$-module. (In fact if $C(\mathcal{B})$ is commutative, $\mathcal{B}$ is an algebra over $C(\mathcal{B})$.) We say that $\mathcal{B}$ is $f g c$ if $\mathcal{B}$ is finitely generated as a $C(\mathcal{B})$-module.

The following lemma is part of [ABFP, Proposition 4.4.5].

Lemma 1.3.7. Suppose that $\Lambda$ is finitely generated and that $\mathcal{B}$ is a graded-centralsimple $\Lambda$-graded algebra so that $\ell \Lambda \subseteq \operatorname{supp}_{\Lambda}(\mathcal{B})$ for some $\ell \geq 1$. Then the following are equivalent:

(a) $\mathcal{B}$ is fgc.

(b) $\mathcal{B}$ is a free module of finite rank over $C(\mathcal{B})$.

(c) $\Lambda / \Gamma(\mathcal{B})$ is finite and $\operatorname{dim}\left(\mathcal{B}^{\lambda}\right)<\infty$ for all $\lambda \in \Lambda$.

\subsection{Fgc centreless Lie tori.}

Proposition 1.4.1. Suppose that $\mathcal{L}$ is a centreless Lie $\Lambda$-torus of type $\Delta$. Then

(i) $\mathcal{L}$ is a graded-central-simple $Q \times \Lambda$-graded and a graded-central-simple $\Lambda$ graded algebra.

(ii) $\Gamma_{Q \times \Lambda}(\mathcal{L})=\{0\} \times \Gamma_{\Lambda}(\mathcal{L})$.

Proof. Let $(\mathfrak{g}, \mathfrak{h})$ be the root-grading pair for $\mathcal{L}$. Yoshii has shown in Y3, Lemma $4.4]$ that $\mathcal{L}$ is a graded-simple $\Lambda$-graded algebra. (The argument uses (9) and (10).) Thus $\mathcal{L}$ is also a graded-simple $Q \times \Lambda$-graded algebra. Consequently the groups $\Gamma_{Q \times \Lambda}(\mathcal{L})$ and $\Gamma_{\Lambda}(\mathcal{L})$ are defined. But since the action of $C(\mathcal{L})$ on $\mathcal{L}$ commutes with the adjoint action of $\mathfrak{h}$, it follows from (9) that $C(\mathcal{L}) \mathcal{L}_{\alpha} \subseteq \mathcal{L}_{\alpha}$ for $\alpha \in Q$. Hence $\operatorname{supp}_{Q \times \Lambda}(C(\mathcal{L})) \subseteq\{0\} \times \Lambda$, so we have (ii). Finally, since $\mathcal{L}$ contains a 1 dimensional homogeneous summand, $\mathcal{L}$ is a graded-central-simple $Q \times \Lambda$-algebra by Remark 1.3.3. Furthermore, using this fact and (ii), we have $C(\mathcal{L})^{0}=C(\mathcal{L})_{0}^{0}=k 1$. Thus $\mathcal{L}$ is a graded-central-simple $\Lambda$-graded algebra. 
For centreless Lie tori, Lemma 1.3.7 simplifies as follows:

Proposition 1.4.2. Suppose that $\Lambda$ is finitely generated, and let $\mathcal{L}$ be a centreless Lie $\Lambda$-torus of type $\Delta$. Then the following statements are equivalent:

(a) $\mathcal{L}$ is fgc.

(b) $\mathcal{L}$ is a free $C(\mathcal{L})$-module of finite rank.

(c) $\Lambda / \Gamma_{\Lambda}(\mathcal{L})$ is finite.

Proof. Now $\mathcal{L}$ is a graded-central-simple algebra by Proposition 1.4.1, and $2 \Lambda \subseteq$ $\operatorname{supp}_{\Lambda}(\mathcal{L})$ by Lemma 1.1.12(vi). Thus, by Lemma 1.3.7, it is enough to show that (c) implies $\operatorname{dim}\left(\mathcal{L}^{\lambda}\right)<\infty$ for $\lambda \in \Lambda$. A stronger result than this (which bounds the root spaces of any extended affine Lie algebra) is announced by Neher in N2, Proposition $3(\mathrm{~b})$ ]. For the convenience of the reader we present the simple argument that is needed here.

Suppose that $\Lambda / \Gamma_{\Lambda}(\mathcal{L})$ is finite. We show that

$$
\operatorname{dim}\left(\mathcal{L}_{\alpha}^{\lambda}\right) \leq \frac{1}{2}\left|\Delta^{\times}\right| s \quad \text { and } \quad \operatorname{dim}\left(\mathcal{L}^{\lambda}\right) \leq \frac{1}{2}\left|\Delta^{\times}\right| s+\left|\Delta^{\times}\right|
$$

for $\alpha \in Q$ and $\lambda \in \Lambda$, where $s=\left|\Lambda / \Gamma_{\Lambda}(\mathcal{L})\right|$. Since $\operatorname{dim}\left(\mathcal{L}_{\alpha}^{\lambda}\right) \leq 1$ for $\alpha \neq 0$, the second equation in (13) follows from the first and it is enough to show the first equation when $\alpha=0$. To do this, let $\Theta$ be a set of representatives of the cosets of $\Gamma_{\Lambda}(\mathcal{L})$ in $\Lambda$. Also let $\Delta_{+}$be the set of positive roots in $\Delta$ relative to some choice of base for $\Delta$. Then for $\lambda \in \Lambda$, we have

$$
\begin{aligned}
\mathcal{L}_{0}^{\lambda} & =\sum_{\alpha \in \Delta_{+}} \sum_{\mu \in \Lambda}\left[\mathcal{L}_{\alpha}^{\mu}, \mathcal{L}_{-\alpha}^{\lambda-\mu}\right] \\
& =\sum_{\alpha \in \Delta_{+}} \sum_{\theta \in \Theta} \sum_{\gamma \in \Gamma_{\Lambda}(\mathcal{L})}\left[\mathcal{L}_{\alpha}^{\gamma+\theta}, \mathcal{L}_{-\alpha}^{\lambda-\gamma-\theta}\right] \\
& =\sum_{\alpha \in \Delta_{+}} \sum_{\theta \in \Theta} \sum_{\gamma \in \Gamma_{\Lambda}(\mathcal{L})}\left[C(\mathcal{L})_{0}^{\gamma} \mathcal{L}_{\alpha}^{\theta}, C(\mathcal{L})_{0}^{-\gamma} \mathcal{L}_{-\alpha}^{\lambda-\theta}\right] \quad \text { (by Lemma 1.3.5) } \\
& =\sum_{\alpha \in \Delta_{+}} \sum_{\theta \in \Theta} \sum_{\gamma \in \Gamma_{\Lambda}(\mathcal{L})} C(\mathcal{L})_{0}^{\gamma} C(\mathcal{L})_{0}^{-\gamma}\left[\mathcal{L}_{\alpha}^{\theta}, \mathcal{L}_{-\alpha}^{\lambda-\theta}\right] \\
& =\sum_{\alpha \in \Delta_{+}} \sum_{\theta \in \Theta}\left[\mathcal{L}_{\alpha}^{\theta}, \mathcal{L}_{-\alpha}^{\lambda-\theta}\right] .
\end{aligned}
$$

Thus we have $\operatorname{dim}\left(\mathcal{L}_{0}^{\lambda}\right) \leq\left|\Delta_{+}\right||\Theta|=\frac{1}{2}\left|\Delta^{\times}\right| s$.

Remark 1.4.3. Suppose that $k$ is algebraically closed (of characteristic 0) and $\Lambda$ is a finitely generated free abelian group of rank $n$. It is a remarkable fact, announced by Neher in [N1, Theorem 7(b)], that if $\Delta$ is not of type $\mathrm{A}_{\ell}$ for $\ell \geq 1$, then any centreless Lie $\Lambda$-torus of type $\Delta$ is fgc. Furthermore, one can see from the classification of centreless Lie $\Lambda$-tori of type $A_{\ell}$ BGK, BGKN, Y1 that there is only one family of centreless Lie $\Lambda$-tori of type $\mathrm{A}_{\ell}$ that are not fgc, namely the family of Lie algebras of the form $\mathrm{sl}_{\ell+1}\left(k_{\mathbf{q}}\right)$, where $k_{\mathbf{q}}$ is the quantum torus associated with an $n \times n$-quantum matrix $\mathbf{q}$ containing an entry that is not a root of unity. Thus in some sense "almost all" centreless Lie tori are fgc. 


\section{BI-ISOMORPHISM AND ISOTOPY FOR LIE TORI}

In this section we introduce and investigate two important notions of isomorphism for Lie tori: bi-isograded-isomorphism, or bi-isomorphism for short, and isotopy. (See also $\mathrm{AF}$ for more information about this topic.)

\subsection{Bi-isomorphism.}

Definition 2.1.1. If $\mathcal{L}$ is a $Q \times \Lambda$-graded algebra and $\mathcal{L}^{\prime}$ is a $Q^{\prime} \times \Lambda^{\prime}$-graded algebra, we say that $\mathcal{L}$ and $\mathcal{L}^{\prime}$ are bi-isograded-isomorphic if there is an algebra isomorphism from $\mathcal{L}$ to $\mathcal{L}^{\prime}$ that is isograded relative to both the root grading and the external grading; this means that there is an algebra isomorphism $\varphi: \mathcal{L} \rightarrow \mathcal{L}^{\prime}$, a group isomorphism $\varphi_{r}: Q \rightarrow Q^{\prime}$, and a group isomorphism $\varphi_{e}: \Lambda \rightarrow \Lambda^{\prime}$ such that

$$
\varphi\left(\mathcal{L}_{\alpha}^{\lambda}\right)=\mathcal{L}_{\varphi_{r}(\alpha)}^{\prime \varphi_{e}(\lambda)}
$$

for $\alpha \in Q$ and $\lambda \in \Lambda$. In that case we call $\varphi$ a bi-isograded-isomorphism. If $\mathcal{L}$ and $\mathcal{L}^{\prime}$ are Lie tori, then since $\left\langle\operatorname{supp}_{Q}(\mathcal{L})\right\rangle=Q$ and $\left\langle\operatorname{supp}_{\Lambda}(\mathcal{L})\right\rangle=\Lambda$, the maps $\varphi_{r}$ and $\varphi_{e}$ are uniquely determined and we call these maps respectively the rootgrading isomorphism and the external-grading isomorphism corresponding to $\varphi$. We will usually abbreviate bi-isograded-isomorphic and bi-isograded-isomorphism as bi-isomorphic and bi-isomorphism, respectively.

Remark 2.1.2. Suppose that $\mathcal{L}$ is a $\Lambda$-torus of type $\Delta$ with root-grading pair $(\mathfrak{g}, \mathfrak{h})$, and $\mathcal{L}^{\prime}$ is a $\Lambda^{\prime}$-torus of type $\Delta^{\prime}$ with root-grading pair $(\mathfrak{g}, \mathfrak{h})$. Suppose that $\varphi$ is a bi-isomorphism of $\mathcal{L}$ onto $\mathcal{L}^{\prime}$. Then it is clear that $\varphi(\mathfrak{g})=\mathfrak{g}^{\prime}$ and $\varphi(\mathfrak{h})=\mathfrak{h}^{\prime}$, so, in this sense, $\mathcal{L}$ and $\mathcal{L}^{\prime}$ have isomorphic root-grading pairs. Also, by the last statement in Lemma 1.1.10, we have

$$
\varphi_{r}\left(\Delta_{\text {ind }}\right)=\Delta_{\text {ind }}
$$

Thus if $\mathcal{L}$ and $\mathcal{L}^{\prime}$ satisfy (LT5), we have $\varphi_{r}(\Delta)=\Delta^{\prime}$, so $\Delta$ and $\Delta^{\prime}$ are isomorphic root systems.

We now see that for the purposes of determining whether or not two Lie tori are bi-isomorphic, we can ignore the root gradings.

Proposition 2.1.3. Suppose that $\mathcal{L}$ is a Lie $\Lambda$-torus of type $\Delta$ and $\mathcal{L}^{\prime}$ is a Lie $\Lambda^{\prime}$-torus of type $\Delta^{\prime}$. Then $\mathcal{L}$ and $\mathcal{L}^{\prime}$ are bi-isomorphic if and only if there is an algebra isomorphism $\varphi: \mathcal{L} \rightarrow \mathcal{L}^{\prime}$ and a group isomorphism $\varphi_{e}: \Lambda \rightarrow \Lambda^{\prime}$ such that $\varphi\left(\mathcal{L}^{\lambda}\right)=\mathcal{L}^{\prime} \varphi_{e}(\lambda)$ for $\lambda \in \Lambda$.

Proof. The implication " $\Rightarrow$ " is immediate from the definition.

Conversely, suppose that we have an isomorphism $\varphi: \mathcal{L} \rightarrow \mathcal{L}^{\prime}$ and a group isomorphism $\varphi_{e}: \Lambda \rightarrow \Lambda^{\prime}$ such that

$$
\varphi\left(\mathcal{L}^{\lambda}\right)=\mathcal{L}^{\prime \varphi_{e}(\lambda)}
$$

for $\lambda \in \Lambda$. Let $Q$ be the root lattice for $\mathcal{L}$ and let $(\mathfrak{g}, \mathfrak{h})$ be the root-grading pair for $\mathcal{L}$. Let $Q^{\prime}$ and $\left(\mathfrak{g}^{\prime}, \mathfrak{h}^{\prime}\right)$ be the corresponding objects for $\mathcal{L}^{\prime}$. By Proposition 1.2 .2 , (iii) and (iv), we may identify $\Delta$ (via the map $\alpha \mapsto \tilde{\alpha}$ ) with a root system in $\mathfrak{h}^{*}$ such that $\Delta_{\text {ind }}$ is the set of roots (including 0 ) of $\mathfrak{g}$ relative to $\mathfrak{h}$ and

$$
\mathcal{L}_{\alpha}=\{x \in \mathcal{L} \mid[h, x]=\alpha(h) x \text { for } h \in \mathfrak{h}\}
$$


for $\alpha \in Q$. Similarly we may identify $\Delta^{\prime}$ with a root system in $\mathfrak{h}^{\prime *}$ such that $\Delta_{\text {ind }}^{\prime}$ is the set of roots (including 0 ) of $\mathfrak{g}^{\prime}$ relative to $\mathfrak{h}^{\prime}$ and

$$
\mathcal{L}_{\alpha^{\prime}}^{\prime}=\left\{x^{\prime} \in \mathcal{L}^{\prime} \mid\left[h^{\prime}, x^{\prime}\right]=\alpha^{\prime}\left(h^{\prime}\right) x^{\prime} \text { for } h^{\prime} \in \mathfrak{h}^{\prime}\right\}
$$

for $\alpha^{\prime} \in Q^{\prime}$. We fix a base for the root system $\Delta$, so we have a notion of positive and negative roots in $\Delta$. Let $\mathfrak{n}_{+}\left(\right.$resp. $\left.\mathfrak{n}_{-}\right)$denote the sum of the root spaces of $\mathfrak{g}$ relative to $\mathfrak{h}$ corresponding to positive (resp. negative) roots. So $\mathfrak{g}$ has the triangular decomposition $\mathfrak{g}=\mathfrak{n}_{+} \oplus \mathfrak{h} \oplus \mathfrak{n}_{-}$.

Observe now that by Proposition 1.2 .2 (vi) we have

$$
\varphi(\mathfrak{g})=\mathfrak{g}^{\prime}
$$

So both $\mathfrak{h}$ and $\varphi^{-1}\left(\mathfrak{h}^{\prime}\right)$ are splitting Cartan subalgebras for $\mathfrak{g}$. Hence, there exists an automorphism $\psi_{\mathfrak{g}}$ of $\mathfrak{g}$ such that $\psi_{\mathfrak{g}}\left(\varphi^{-1}\left(\mathfrak{h}^{\prime}\right)\right)=\mathfrak{h}$. In fact, by [Sel, Corollary on p. 28], $\psi_{\mathfrak{g}}$ can be chosen of the form

$$
\psi_{\mathfrak{g}}=\exp \left(\operatorname{ad}_{\mathfrak{g}}\left(x_{1}\right)\right) \ldots \exp \left(\operatorname{ad}_{\mathfrak{g}}\left(x_{\ell}\right)\right),
$$

where $x_{i} \in \mathfrak{n}_{-} \cup \mathfrak{n}_{+}$for $1 \leq i \leq \ell$. Now since $x_{i} \in \mathfrak{n}_{-} \cup \mathfrak{n}_{+}$, it follows that $\operatorname{ad}_{\mathcal{L}}\left(x_{i}\right)$ is nilpotent for $1 \leq i \leq \ell$. So we may define

$$
\psi_{\mathcal{L}}=\exp \left(\operatorname{ad}_{\mathcal{L}}\left(x_{1}\right)\right) \ldots \exp \left(\operatorname{ad}_{\mathcal{L}}\left(x_{\ell}\right)\right) \in \operatorname{Aut}_{k}(\mathcal{L}) .
$$

Then $\psi_{\mathcal{L}}$ extends $\psi_{\mathfrak{g}}$, so

$$
\psi_{\mathcal{L}}\left(\varphi^{-1}\left(\mathfrak{h}^{\prime}\right)\right)=\mathfrak{h} .
$$

Also since $x_{i} \in \mathfrak{g} \subseteq \mathcal{L}^{0}$ for each $i$, we have $\psi_{\mathcal{L}}\left(\mathcal{L}^{\lambda}\right) \subseteq \mathcal{L}^{\lambda}$ for $\lambda \in \Lambda$. Therefore since $\psi_{\mathcal{L}} \in \operatorname{Aut}_{k}(\mathcal{L})$ we have $\psi_{\mathcal{L}}\left(\mathcal{L}^{\lambda}\right)=\mathcal{L}^{\lambda}$ for $\lambda \in \Lambda$. Thus replacing $\varphi$ by $\varphi \circ\left(\psi_{\mathcal{L}}\right)^{-1}$ we may assume that

$$
\varphi(\mathfrak{h})=\mathfrak{h}^{\prime} .
$$

If $\alpha \in \mathfrak{h}^{*}$, we define $\mathcal{L}_{\alpha}$ by the equality (14). Similarly, if $\alpha^{\prime} \in \mathfrak{h}^{\prime *}$ we define $\mathcal{L}_{\alpha^{\prime}}^{\prime}$ by the equality (15). Also, let $\hat{\varphi}: \mathfrak{h}^{*} \rightarrow \mathfrak{h}^{\prime *}$ denote the inverse dual of the linear map $\left.\varphi\right|_{\mathfrak{h}}: \mathfrak{h} \rightarrow \mathfrak{h}^{\prime}$; that is, $\hat{\varphi}$ is defined by $\hat{\varphi}(\alpha)\left(h^{\prime}\right)=\alpha\left(\varphi^{-1}\left(h^{\prime}\right)\right)$ for $\alpha \in \mathfrak{h}^{*}$ and $h^{\prime} \in \mathfrak{h}^{\prime}$. Then it follows from (16) that

$$
\varphi\left(\mathcal{L}_{\alpha}\right)=\mathcal{L}_{\hat{\varphi}(\alpha)}^{\prime}
$$

for $\alpha \in \mathfrak{h}^{*}$. Consequently, we have $\hat{\varphi}\left(\operatorname{supp}_{Q}(\mathcal{L})\right)=\operatorname{supp}_{Q^{\prime}}\left(\mathcal{L}^{\prime}\right)$. But by Lemma 1.1.10 we have $\operatorname{span}_{\mathbb{Z}}\left(\operatorname{supp}_{Q}(\mathcal{L})\right)=Q$ and $\operatorname{span}_{\mathbb{Z}}\left(\operatorname{supp}_{Q^{\prime}}\left(\mathcal{L}^{\prime}\right)\right)=Q^{\prime}$. Therefore $\hat{\varphi}(Q)=Q^{\prime}$, and we may define $\varphi_{r}=\left.\hat{\varphi}\right|_{Q}: Q \rightarrow Q^{\prime}$. Then for $\alpha \in Q$ and $\lambda \in \Lambda$, we have

$$
\varphi\left(\mathcal{L}_{\alpha}^{\lambda}\right)=\varphi\left(\mathcal{L}_{\alpha} \cap \mathcal{L}^{\lambda}\right)=\varphi\left(\mathcal{L}_{\alpha}\right) \cap \varphi\left(\mathcal{L}^{\lambda}\right)=\mathcal{L}_{\varphi_{r}(\alpha)}^{\prime} \cap \mathcal{L}^{\prime \varphi_{e}(\lambda)}=\mathcal{L}_{\varphi_{r}(\alpha)}^{\prime \varphi_{e}(\lambda)} .
$$

2.2. Isotopes and isotopy. Suppose in this subsection that $\mathcal{L}$ is a Lie $\Lambda$-torus of type $\Delta$, and let $Q$ be the root lattice of $\Delta$.

Definition 2.2.1. Suppose that $s \in \operatorname{Hom}(Q, \Lambda)$, where $\operatorname{Hom}(Q, \Lambda)$ is the group of group homomorphisms from $Q$ into $\Lambda$. We define a new $Q \times \Lambda$-graded Lie algebra $\mathcal{L}^{(s)}$ as follows. As a Lie algebra, $\mathcal{L}^{(s)}=\mathcal{L}$. The grading on $\mathcal{L}^{(s)}$ is given by

$$
\left(\mathcal{L}^{(s)}\right)_{\alpha}^{\lambda}=\mathcal{L}_{\alpha}^{\lambda+s(\alpha)}
$$

for $\alpha \in Q, \lambda \in \Lambda$. 
Remark 2.2.2. Let $s \in \operatorname{Hom}(Q, \Lambda)$.

(i) Observe that $\left(\mathcal{L}^{(s)}\right)_{\alpha}=\mathcal{L}_{\alpha}$ for $\alpha \in Q$, and hence $\operatorname{supp}_{Q}\left(\mathcal{L}^{(s)}\right)=\operatorname{supp}_{Q}(\mathcal{L})$.

(ii) Recall that for $\alpha \in Q$ we have defined $\Lambda_{\alpha}=\left\{\lambda \in \Lambda \mid \mathcal{L}_{\alpha}^{\lambda} \neq 0\right\}$ (see (6)). Then, if $\alpha \in Q$, we have $\left(\mathcal{L}^{(s)}\right)_{\alpha}^{0}=\mathcal{L}_{\alpha}^{s(\alpha)}$, so

$$
\left(\mathcal{L}^{(s)}\right)_{\alpha}^{0} \neq 0 \Longleftrightarrow s(\alpha) \in \Lambda_{\alpha} .
$$

Proposition 2.2.3. Let $s \in \operatorname{Hom}(Q, \Lambda)$, and let $\Pi$ be a base for the root system $\Delta$. The following statements are equivalent:

(a) $\mathcal{L}^{(s)}$ is a Lie torus.

(b) $s(\alpha) \in \Lambda_{\alpha}$ for all $\alpha \in \Delta_{\text {ind }}^{\times}$.

(c) $s(\alpha) \in \Lambda_{\alpha}$ for all $\alpha \in \Pi$.

Moreover, if these conditions are satisfied, then $\mathcal{L}^{(s)}$ satisfies (LT5) if and only if $\mathcal{L}$ satisfies (LT5).

Proof. The final statement is clear using Remark 2.2.2(i). So we need to prove the equivalence of (a), (b) and (c). To simplify notation, let $\widetilde{\mathcal{L}}=\mathcal{L}^{(s)}$.

"(a) $\Leftrightarrow(\mathrm{b})$ " If (a) holds, then $\widetilde{\mathcal{L}}$ satisfies (LT2)(i), and hence, by (18), (b) holds. For the converse, suppose that (b) holds. Since $\mathcal{L}$ satisfies (LT1) and (LT3), so does $\widetilde{\mathcal{L}}$ (by Remark [2.2.2(i)). Also, it follows from (b) and (18) that $\widetilde{\mathcal{L}}$ satisfies (LT2)(i). To show that $\widetilde{\mathcal{L}}$ satisfies (LT2)(ii), let $(\alpha, \lambda) \in \operatorname{supp}_{Q \times \Lambda}(\widetilde{\mathcal{L}})$ and $\alpha \in$ $\Delta^{\times}$. Then $(\alpha, \lambda+s(\alpha)) \in \operatorname{supp}_{Q \times \Lambda}(\mathcal{L})$, so we can choose $e_{\alpha}^{\lambda+s(\alpha)} \in \mathcal{L}_{\alpha}^{\lambda+s(\alpha)}$ and $f_{\alpha}^{\lambda+s(\alpha)} \in \mathcal{L}_{-\alpha}^{-\lambda-s(\alpha)}$ as in (LT2)(ii) (for $\left.\mathcal{L}\right)$. Set $\tilde{e}_{\alpha}^{\lambda}=e_{\alpha}^{\lambda+s(\alpha)}$ and $\tilde{f}_{\alpha}^{\lambda}=f_{\alpha}^{\lambda+s(\alpha)}$. Then $\widetilde{\mathcal{L}}_{\alpha}^{\lambda}=k \tilde{e}_{\alpha}^{\lambda}, \widetilde{\mathcal{L}}_{-\alpha}^{-\lambda}=k \tilde{f}_{\alpha}^{\lambda}$, and

$$
\left[\left[\tilde{e}_{\alpha}^{\lambda}, \tilde{f}_{\alpha}^{\lambda}\right], x_{\beta}\right]=\left[\left[e_{\alpha}^{\lambda+s(\alpha)}, f_{\alpha}^{\lambda+s(\alpha)}\right], x_{\beta}\right]=\left\langle\beta, \alpha^{\vee}\right\rangle x_{\beta}
$$

for $x_{\beta} \in \widetilde{\mathcal{L}}_{\beta}=\mathcal{L}_{\beta}, \beta \in Q$. Thus, $\widetilde{\mathcal{L}}$ satisfies (LT2)(ii). To show that $\widetilde{\mathcal{L}}$ satisfies (LT4), let

$$
\widetilde{\Lambda}_{\alpha}=\left\{\lambda \in \Lambda \mid \widetilde{\mathcal{L}}_{\alpha}^{\lambda} \neq 0\right\}=\Lambda_{\alpha}-s(\alpha)
$$

for $\alpha \in Q$. Choose $\beta \in \Delta_{\text {sh }}$. Then, by Lemma 1.1.12 (v), $2 \Lambda \subseteq \Lambda_{\beta}$. So, if $\lambda \in \Lambda_{\beta}$, we have $\lambda=(\lambda-s(\beta))+(2 s(\beta)-s(\beta)) \in\left(\Lambda_{\beta}-s(\beta)\right)+\left(\Lambda_{\beta}-s(\beta)\right) \in \widetilde{\Lambda}_{\beta}+\widetilde{\Lambda}_{\beta}$. So $\Lambda_{\beta} \subseteq \tilde{\Lambda}_{\beta}+\tilde{\Lambda}_{\beta}$. But, by Lemma 1.1.12(v), we have $\left\langle\Lambda_{\beta}\right\rangle=\Lambda$. So $\left\langle\tilde{\Lambda}_{\beta}\right\rangle=\Lambda$ and hence $\operatorname{supp}_{\Lambda}(\widetilde{\mathcal{L}})=\widetilde{\mathcal{L}}$. Therefore $\widetilde{\mathcal{L}}$ satisfies (LT4) and we have (a).

"(b) $\Leftrightarrow(\mathrm{c})$ " The implication "(b) $\Rightarrow(\mathrm{c})$ " is trivial. For the converse we assume that (c) holds. Let $\gamma \in \Delta_{\text {ind }}^{\times}$. Then $\gamma=w_{\alpha_{1}} \ldots w_{\alpha_{p}} \alpha_{p+1}$ for some $\alpha_{1}, \ldots, \alpha_{p}, \alpha_{p+1} \in \Pi$. Let $g=g_{1} \ldots g_{p}$, where

$$
g_{i}=\theta_{\alpha_{i}}^{s\left(\alpha_{i}\right)}=\exp \left(\operatorname{ad}\left(e_{\alpha_{i}}^{s\left(\alpha_{i}\right)}\right)\right) \exp \left(\operatorname{ad}\left(-f_{\alpha_{i}}^{s\left(\alpha_{i}\right)}\right)\right) \exp \left(\operatorname{ad}\left(e_{\alpha_{i}}^{s\left(\alpha_{i}\right)}\right)\right)
$$

for $1 \leq i \leq p$. Then, by (4), $g\left(\mathcal{L}_{\alpha_{p+1}}\right)=\mathcal{L}_{\gamma}$, so $g\left(\widetilde{\mathcal{L}}_{\alpha_{p+1}}\right)=\widetilde{\mathcal{L}}_{\gamma}$. But $e_{\alpha_{i}}^{s\left(\alpha_{i}\right)}$ and $f_{\alpha_{i}}^{s\left(\alpha_{i}\right)}$ are in $\widetilde{\mathcal{L}}^{0}$ for $1 \leq i \leq p$, so $g\left(\widetilde{\mathcal{L}}^{0}\right) \subseteq \widetilde{\mathcal{L}}^{0}$. Therefore, $g\left(\widetilde{\mathcal{L}}_{\alpha_{p+1}}^{0}\right) \subseteq g\left(\widetilde{\mathcal{L}}_{\gamma}^{0}\right)$. Hence, since $\widetilde{\mathcal{L}}_{\alpha_{p+1}}^{0} \neq 0$, we have $\widetilde{\mathcal{L}}_{\gamma}^{0} \neq 0$. So $s(\gamma) \in \Lambda_{\gamma}$, and (b) holds.

Definition 2.2.4. Suppose that $s \in \operatorname{Hom}(Q, \Lambda)$. If $s$ satisfies the conditions (a)(c) in Proposition 2.2.3 we say that $s$ is admissible for $\mathcal{L}$. In that case, we call the Lie torus $\mathcal{L}^{(s)}$ the s-isotope of $\mathcal{L}$. 
Remark 2.2.5. There are coordinatization theorems for the centreless Lie tori of each type (as mentioned in the introduction), and the notion of isotope for a centreless Lie torus corresponds in several cases to a corresponding classical notion of isotope for the coordinate algebra $\mathrm{AF}$. To give one example, Yoshii has shown that every centreless Lie torus of type $A_{1}$ is isomorphic to the Tits-Kantor-Koecher Lie algebra $\operatorname{TKK}(\mathcal{J})$ constructed from a Jordan torus $\mathcal{J}[\mathrm{Y} 1$. One can easily show that the isotopes of the Lie torus $\operatorname{TKK}(\mathcal{J})$ are up to bi-isomorphism the Lie tori $\operatorname{TKK}\left(\mathcal{J}^{(u)}\right)$, where $u$ runs over all nonzero homogeneous (hence invertible) elements of $\mathcal{J}$ and where $\mathcal{J}^{(u)}$ is the $u$-isotope of $\mathcal{J}$ [AF, Prop. 8.5]. (See for example [Mc, $\left.\S 7.2\right]$ for information about isotopes of Jordan algebras.)

Remark 2.2.6. Suppose that $\Pi=\left\{\alpha_{1}, \ldots, \alpha_{r}\right\}$ is a base for $\Delta$. By Proposition2.2.3. to specify an admissible $s \in \operatorname{Hom}(Q, \Lambda)$ for $\mathcal{L}$, and hence an isotope $\mathcal{L}^{(s)}$ of $\mathcal{L}$, one can arbitrarily choose $\lambda_{i} \in \Lambda_{\alpha_{i}}$ for $1 \leq i \leq r$, and then define $s \in \operatorname{Hom}(Q, \Lambda)$ with $s\left(\alpha_{i}\right)=\lambda_{i}$ for $1 \leq i \leq r$.

Lemma 2.2.7. Suppose that $s \in \operatorname{Hom}(Q, \Lambda)$ is admissible for $\mathcal{L}$. Then:

(i) $\mathcal{L}^{(0)}=\mathcal{L}$.

(ii) If $t \in \operatorname{Hom}(Q, \Lambda)$, then $t$ is admissible for $\mathcal{L}^{(s)}$ if and only if $s+t$ is admissible for $\mathcal{L}$. Moreover, in that case, $\left(\mathcal{L}^{(s)}\right)^{(t)}=\mathcal{L}^{(s+t)}$.

(iii) $-s$ is admissible for $\mathcal{L}^{(s)}$ and $\left(\mathcal{L}^{(s)}\right)^{(-s)}=\mathcal{L}$.

Proof. (i) is clear. For (ii), we write $\Lambda_{\alpha}^{(s)}:=\left\{\lambda \in \Lambda:\left(\mathcal{L}^{(s)}\right)_{\alpha}^{\lambda} \neq 0\right\}=\Lambda_{\alpha}-s(\alpha)$ for $\alpha \in Q$. Then,

$$
\begin{aligned}
t \text { is admissible for } \mathcal{L}^{(s)} & \Longleftrightarrow t(\alpha) \in \Lambda_{\alpha}^{(s)} \text { for } \alpha \in \Delta_{\text {ind }}^{\times} \\
& \Longleftrightarrow t(\alpha) \in \Lambda_{\alpha}-s(\alpha) \text { for } \alpha \in \Delta_{\text {ind }}^{\times} \\
& \Longleftrightarrow(s+t)(\alpha) \in \Lambda_{\alpha} \text { for } \alpha \in \Delta_{\text {ind }}^{\times} \\
& \Longleftrightarrow s+t \text { is admissible for } \mathcal{L} .
\end{aligned}
$$

The second statement in (ii) is clear. Finally, (iii) follows from (i) and (ii).

The following lemma is easily checked.

Lemma 2.2.8. Suppose that $\mathcal{L}$ is a Lie $\Lambda$-torus of type $\Delta, \mathcal{L}^{\prime}$ is a Lie $\Lambda^{\prime}$-torus of type $\Delta^{\prime}$, and $\varphi$ is a bi-isomorphism of $\mathcal{L}$ onto $\mathcal{L}^{\prime}$ (see Definition 2.1.1). Suppose that $s \in \operatorname{Hom}(Q, \Lambda)$ and $s^{\prime} \in \operatorname{Hom}\left(Q^{\prime}, \Lambda^{\prime}\right)$ with $\varphi_{e} s=s^{\prime} \varphi_{r}$. Then $s$ is admissible for $\mathcal{L}$ if and only if $s^{\prime}$ is admissible for $\mathcal{L}^{\prime}$. Moreover, in that case, $\varphi$ is a biisomorphism of $\mathcal{L}^{(s)}$ onto $\mathcal{L}^{\prime\left(s^{\prime}\right)}$ (with the same root-grading isomorphism and the same external-grading isomorphism).

Definition 2.2.9. Suppose that $\mathcal{L}$ is a Lie $\Lambda$-torus of type $\Delta$ and $\mathcal{L}^{\prime}$ is a Lie $\Lambda^{\prime}$ torus of type $\Delta^{\prime}$. We say that $\mathcal{L}$ is isotopic to $\mathcal{L}^{\prime}$, written $\mathcal{L} \sim \mathcal{L}^{\prime}$, if some isotope $\mathcal{L}^{(s)}$ of $\mathcal{L}$ is bi-isomorphic to $\mathcal{L}^{\prime}$.

It follows easily from Lemmas 2.2 .7 and 2.2 .8 that $\sim$ is an equivalence relation on the class of Lie tori.

\section{Multiloop Realization}

In the rest of the paper we investigate multiloop realizations of centreless Lie tori of nullity $n$. If $n=0$, any such Lie torus is a finite dimensional split simple Lie 
algebra (see Remark 1.2.4), and we do not have anything to add to the theory of these algebras. Thus, for the rest of the paper we assume that $n$ is an integer $\geq 1$.

We also suppose for the rest of the paper that $k$ is algebraically closed (of characteristic 0$)$. Thus, $k^{\times}$contains a primitive $\ell^{\text {th }}$ root of unity $\zeta_{\ell}$ for all positive integers $\ell$. We assume that we have made a fixed compatible choice of these roots of unity in $k^{\times}$in the sense that

$$
\zeta_{m \ell}^{m}=\zeta_{\ell}
$$

for all $\ell, m \geq 1$.

3.1. Multiloop algebras. We first recall the definitions and the results that we will need from $\mathrm{ABFP}$ regarding multiloop algebras in general. In order to do this conveniently we first introduce some notation.

\section{Notation 3.1.1.}

(i) Suppose that $G$ is a group. We let

$$
\operatorname{cfo}_{n}(G)=\left\{\boldsymbol{\sigma}=\left(\sigma_{1}, \ldots, \sigma_{n}\right) \in G^{n}|| \sigma_{i} \mid<\infty, \sigma_{i} \sigma_{j}=\sigma_{j} \sigma_{i} \text { for all } i, j\right\}
$$

denote the set of all length $n$ sequences of commuting finite order elements of elements of $G$. If $P=\left(p_{i j}\right) \in \operatorname{Mat}_{n}(\mathbb{Z})$ and $\boldsymbol{\sigma}=\left(\sigma_{1}, \ldots, \sigma_{n}\right) \in \operatorname{cfo}_{n}(G)$, we set

$$
\boldsymbol{\sigma}^{P}=\left(\prod_{i=1}^{n} \sigma_{i}^{p_{i 1}}, \ldots, \prod_{i=1}^{n} \sigma_{i}^{p_{i n}}\right) \in \operatorname{cfo}_{n}(G)
$$

One sees that

$$
\left(\sigma^{P}\right)^{P^{\prime}}=\sigma^{P P^{\prime}}
$$

for $P, P^{\prime} \in \operatorname{Mat}_{n}(\mathbb{Z})$. In particular, this gives a right action of $\mathrm{GL}_{n}(\mathbb{Z})$ on $\operatorname{cfo}_{n}(G)$. If $\boldsymbol{\sigma} \in \operatorname{cfo}_{n}(G)$, we let

$$
\langle\boldsymbol{\sigma}\rangle=\left\langle\sigma_{1}, \ldots, \sigma_{n}\right\rangle
$$

denote the subgroup of $G$ generated by $\sigma_{1}, \ldots, \sigma_{n}$. Then, if $P \in \operatorname{Mat}_{n}(\mathbb{Z})$, it is clear that $\left\langle\boldsymbol{\sigma}^{P}\right\rangle \subseteq\langle\boldsymbol{\sigma}\rangle$; so if $P \in \mathrm{GL}_{n}(\mathbb{Z})$, we have

$$
\left\langle\boldsymbol{\sigma}^{P}\right\rangle=\langle\boldsymbol{\sigma}\rangle \text {. }
$$

Let

$$
\mathbf{1}=(1, \ldots, 1) .
$$

in $G^{n}$. Then if $\boldsymbol{\sigma} \in \operatorname{cfo}_{n}(G)$ and $\mathbf{m} \in \mathbb{Z}_{+}^{n}$, where $\mathbb{Z}_{+}$denotes the set of positive integers, we write

$$
\sigma^{\mathrm{m}}=1
$$

to mean that $\sigma_{i}^{m_{i}}=1$ for all $i$.

(ii) If $\mathcal{A}$ is an algebra, we use the notation in (i) for the group $G=\operatorname{Aut}_{k}(\mathcal{A})$. For brevity we write

$$
\operatorname{cfo}_{n}(\mathcal{A})=\operatorname{cfo}_{n}\left(\operatorname{Aut}_{k}(\mathcal{A})\right) .
$$

That is, $\operatorname{cfo}_{n}(\mathcal{A})$ is the set of length $n$ sequences of commuting finite order automorphisms of $\mathcal{A}$. We have a right action of $\mathrm{GL}_{n}(\mathbb{Z})$ on $\operatorname{cfo}_{n}(\mathcal{A})$ as in (i).

(iii) If $\varphi: \mathcal{A} \rightarrow \mathcal{A}^{\prime}$ is an isomorphism of algebras and $\boldsymbol{\sigma}=\left(\sigma_{1}, \ldots, \sigma_{n}\right) \in \operatorname{cfo}_{n}(\mathcal{A})$, we set

$$
\varphi \boldsymbol{\sigma} \varphi^{-1}=\left(\varphi \sigma_{1} \varphi^{-1}, \ldots, \varphi \sigma_{n} \varphi^{-1}\right) \in \operatorname{cfo}_{n}\left(\mathcal{A}^{\prime}\right)
$$


Definition 3.1.2. Suppose $\mathcal{A}$ is an (ungraded) algebra over $k, \boldsymbol{\sigma}=\left(\sigma_{1}, \ldots, \sigma_{n}\right) \in$ $\operatorname{cfo}_{n}(\mathcal{A})$, and $\mathbf{m}=\left(m_{1}, \ldots, m_{n}\right) \in \mathbb{Z}_{+}^{n}$ with $\boldsymbol{\sigma}^{\mathbf{m}}=\mathbf{1}$. Let $\bar{\Lambda}=\mathbb{Z} /\left(m_{1}\right) \oplus \cdots \oplus \mathbb{Z} /\left(m_{n}\right)$ and let $\lambda=\left(\ell_{1}, \ldots, \ell_{n}\right) \mapsto \bar{\lambda}=\left(\bar{\ell}_{1}, \ldots, \bar{\ell}_{n}\right)$ be the canonical group homomorphism from $\mathbb{Z}^{n}$ onto $\bar{\Lambda}$, where $\bar{\ell}_{j}=\ell_{j}+m_{j} \mathbb{Z}$ for each $j$. We give the algebra $\mathcal{A}$ a $\bar{\Lambda}$-grading, called the $\bar{\Lambda}$-grading of $\mathcal{A}$ determined by $\boldsymbol{\sigma}$, by setting

$$
\mathcal{A}^{\bar{\lambda}}=\left\{u \in \mathcal{A} \mid \sigma_{j} u=\zeta_{m_{j}}^{\ell_{j}} u \text { for } 1 \leq j \leq n\right\}
$$

for $\bar{\lambda}=\left(\bar{\ell}_{1}, \ldots, \bar{\ell}_{n}\right) \in \bar{\Lambda}$. Let $k\left[z_{1}^{ \pm 1}, \ldots, z_{n}^{ \pm 1}\right]=\sum_{\lambda \in \mathbb{Z}^{n}} k z^{\lambda}$ be the algebra of Laurent polynomials over $k$, where $z^{\lambda}=z_{1}^{\ell_{1}} \ldots z_{n}^{\ell_{n}}$ for $\lambda=\left(\ell_{1}, \ldots, \ell_{n}\right) \in \mathbb{Z}^{n}$. Let

$$
M_{\mathbf{m}}(\mathcal{A}, \boldsymbol{\sigma})=\sum_{\lambda \in \mathbb{Z}^{n}} \mathcal{A}^{\bar{\lambda}} \otimes z^{\lambda}
$$

in the algebra $\mathcal{A} \otimes_{k} k\left[z_{1}^{ \pm 1}, \ldots, z_{n}^{ \pm 1}\right]$. Then $M_{\mathbf{m}}(\mathcal{A}, \boldsymbol{\sigma})$ is a subalgebra of $\mathcal{A} \otimes_{k}$ $k\left[z_{1}^{ \pm 1}, \ldots, z_{n}^{ \pm 1}\right]$. We define a $\mathbb{Z}^{n}$-grading on $M_{\mathbf{m}}(\mathcal{A}, \boldsymbol{\sigma})$ by setting

$$
M_{\mathbf{m}}(\mathcal{A}, \boldsymbol{\sigma})^{\lambda}=\mathcal{A}^{\bar{\lambda}} \otimes z^{\lambda}
$$

for all $\lambda \in \mathbb{Z}^{n}$. We call the $\mathbb{Z}^{n}$-graded algebra $M_{\mathbf{m}}(\mathcal{A}, \boldsymbol{\sigma})$ the multiloop algebra of $\boldsymbol{\sigma}$ (based on $\mathcal{A}$ and relative to $\mathbf{m}$ ).

By ABFP, Lemma 3.2.4], we have the following:

Lemma 3.1.3. Suppose that $\mathbf{m}, \mathcal{A}$ and $\boldsymbol{\sigma}$ are as in Definition 3.1.2, Then

$$
\left\langle\operatorname{supp}_{\mathbb{Z}^{n}}\left(M_{\mathbf{m}}(\mathcal{A}, \boldsymbol{\sigma})\right)\right\rangle=\mathbb{Z}^{n} \Longleftrightarrow\left|\left\langle\sigma_{1}, \ldots, \sigma_{n}\right\rangle\right|=m_{1} \cdots m_{n} .
$$

The following result, which is part of [ABFP, Corollary 8.3.5], tells us which graded algebras have multiloop realizations based on finite dimensional simple algebras.

Theorem 3.1.4. Let $\mathcal{B}$ be a $\Lambda$-graded algebra, where $\Lambda$ is a free abelian group of finite rank $n \geq 1$. Then $\mathcal{B}$ is isograded-isomorphic to $M_{\mathbf{m}}(\mathcal{A}, \boldsymbol{\sigma})$ for some finite dimensional simple (ungraded) algebra $\mathcal{A}$, some $\boldsymbol{\sigma} \in \operatorname{cfo}_{n}(\mathcal{A})$ and some $\mathbf{m} \in \mathbb{Z}_{+}^{n}$ with $\boldsymbol{\sigma}^{\mathbf{m}}=\mathbf{1}$ if and only if $\mathcal{B}$ is graded-central-simple, $\mathcal{B}$ is fgc and $\Gamma(\mathcal{B})$ has finite index in $\Lambda$.

We also have necessary and sufficient conditions for isograded-isomorphism of multiloop algebras $M_{\mathbf{m}}(\mathcal{A}, \boldsymbol{\sigma})$ that satisfy $\left\langle\operatorname{supp}_{\mathbb{Z}^{n}}\left(M_{\mathbf{m}}(\mathcal{A}, \boldsymbol{\sigma})\right)=\mathbb{Z}^{n}\right.$.

Theorem 3.1.5. Suppose $\mathcal{A}$ is an (ungraded) central-simple algebra over $k, \boldsymbol{\sigma}=$ $\left(\sigma_{1}, \ldots, \sigma_{n}\right) \in \operatorname{cfo}_{n}(\mathcal{A}), \mathbf{m}=\left(m_{1}, \ldots, m_{n}\right) \in \mathbb{Z}_{+}^{n}$ with $\boldsymbol{\sigma}^{\mathbf{m}}=\mathbf{1}$, and $\left|\left\langle\sigma_{1}, \ldots, \sigma_{n}\right\rangle\right|=$ $m_{1} \cdots m_{n}$. Suppose also that $\mathbf{m}^{\prime}, \mathcal{A}^{\prime}$ and $\boldsymbol{\sigma}^{\prime}$ satisfy the same assumptions. Then $M_{\mathbf{m}}(\mathcal{A}, \boldsymbol{\sigma})$ is isograded-isomorphic to $M_{\mathbf{m}^{\prime}}\left(\mathcal{A}^{\prime}, \boldsymbol{\sigma}^{\prime}\right)$ if and only if there exists a matrix $P=\left(p_{i j}\right) \in \mathrm{GL}_{n}(\mathbb{Z})$ and an algebra isomorphism $\varphi: \mathcal{A} \rightarrow \mathcal{A}^{\prime}$ such that

$$
\boldsymbol{\sigma}^{\prime}=\varphi \boldsymbol{\sigma}^{P} \varphi^{-1} .
$$

Proof. Note that by Lemma 3.1 .3 we have $\left\langle\operatorname{supp}_{\mathbb{Z}^{n}}\left(M_{\mathbf{m}}(\mathcal{A}, \boldsymbol{\sigma})\right)\right\rangle=\mathbb{Z}^{n}$ as well as the corresponding primed statement. The theorem then is the second statement of ABFP, Theorem 8.3.2(ii)].

Remark 3.1.6. The action $(\sigma, P) \mapsto \sigma^{P}$ of $\mathrm{GL}(n, \mathbb{Z})$ on $\operatorname{cfo}_{n}(\mathcal{A})$ also plays an important role in the study of the isomorphism problem for multiloop algebras regarded as (ungraded) algebras GP, Lemma 5.3]. 
3.2. The construction of multiloop Lie tori. We next want to describe a multiloop construction of a centreless Lie torus $\operatorname{LT}(\mathfrak{s}, \sigma, \mathfrak{h})$ that we will call a multiloop Lie $\mathbb{Z}^{n}$-torus. For technical reasons we begin with a more general construction of a $Q \times \mathbb{Z}^{n}$-graded Lie algebra that we denote by $\operatorname{LT}_{\mathbf{m}}(\mathfrak{s}, \sigma, \mathfrak{h})$. (LT in this notation is once again an abbreviation for Lie torus.)

If $\mathfrak{s}$ is an algebra and $\boldsymbol{\sigma}=\left(\sigma_{1}, \ldots, \sigma_{n}\right) \in \operatorname{cfo}_{n}(\mathfrak{s})$, we let

$$
\mathfrak{s}^{\boldsymbol{\sigma}}=\left\{x \in \mathfrak{s} \mid \sigma_{i}(x)=x \text { for } 1 \leq i \leq n\right\}
$$

denote the fixed point subalgebra for $\langle\boldsymbol{\sigma}\rangle$ in $\mathfrak{s}$.

Construction 3.2.1. Let $\mathfrak{s}$ be a finite dimensional simple Lie algebra over $k$; let $\boldsymbol{\sigma}=\left(\sigma_{1}, \ldots, \sigma_{n}\right) \in \operatorname{cfo}_{n}(\mathfrak{s})$; let $\mathfrak{h}$ be an ad-diagonalizable subalgebra of $\mathfrak{s}$ that is contained in $\mathfrak{s}^{\boldsymbol{\sigma}}$; and let $\mathbf{m}=\left(m_{1}, \ldots, m_{n}\right) \in \mathbb{Z}_{+}^{n}$ with $\boldsymbol{\sigma}^{\mathbf{m}}=\mathbf{1}$. We now construct a $Q \times \mathbb{Z}^{n}$-graded algebra $\operatorname{LT}_{\mathbf{m}}(\mathfrak{s}, \boldsymbol{\sigma}, \mathfrak{h})$ from this data, where

$$
\Delta=\Delta(\mathfrak{s}, \mathfrak{h}) \quad \text { and } \quad Q=\operatorname{span}_{\mathbb{Z}}(\Delta) .
$$

(However, with our assumptions so far, $\Delta$ is not necessarily a root system.)

Set $\bar{\Lambda}=\mathbb{Z} /\left(m_{1}\right) \oplus \cdots \oplus \mathbb{Z} /\left(m_{n}\right)$. Then $\mathfrak{s}$ is $Q$-graded using the root space decomposition of $\mathfrak{s}$ relative to the adjoint action of $\mathfrak{h}$ (see Definition 1.1.4), and $\mathfrak{s}$ is $\bar{\Lambda}$-graded using the $\bar{\Lambda}$-grading determined by $\boldsymbol{\sigma}$ (see Definition 3.1.2). These gradings are compatible and so they determine a $Q \times \bar{\Lambda}$-grading $\mathfrak{s}=\bigoplus_{(\alpha, \bar{\lambda}) \in Q \times \bar{\Lambda}} \mathfrak{s}_{\alpha}^{\bar{\lambda}}$ on $\mathfrak{s}$. Define the algebra

$$
\operatorname{LT}_{\mathbf{m}}(\mathfrak{s}, \boldsymbol{\sigma}, \mathfrak{h})=\sum_{\lambda \in \mathbb{Z}^{n}} \mathfrak{s}^{\bar{\lambda}} \otimes z^{\lambda}
$$

in $\mathfrak{s} \otimes k\left[z_{1}^{ \pm 1}, \ldots, z_{n}^{ \pm 1}\right]$, and give this algebra a $Q \times \mathbb{Z}^{n}$-grading by setting

$$
\operatorname{LT}_{\mathbf{m}}(\mathfrak{s}, \boldsymbol{\sigma}, \mathfrak{h})_{\alpha}^{\lambda}=\mathfrak{s}_{\alpha}^{\bar{\lambda}} \otimes z^{\lambda}
$$

for $\alpha \in Q$ and $\lambda \in \mathbb{Z}^{n}$. Thus, the $Q$-grading on $\operatorname{LT}_{\mathbf{m}}(\mathfrak{s}, \boldsymbol{\sigma}, \mathfrak{h})$ is the root space decomposition of $\operatorname{LT}_{\mathbf{m}}(\mathfrak{s}, \boldsymbol{\sigma}, \mathfrak{h})$ relative to the ad-diagonalizable subalgebra $\mathfrak{h} \otimes 1$. As a $\mathbb{Z}^{n}$-graded algebra, $\operatorname{LT}_{\mathbf{m}}(\mathfrak{s}, \boldsymbol{\sigma}, \mathfrak{h})$ is the multiloop algebra $M_{\mathbf{m}}(\mathfrak{s}, \boldsymbol{\sigma})$ defined in Definition 3.1.2.

Remark 3.2.2. Suppose that $\mathfrak{s}, \boldsymbol{\sigma}, \mathfrak{h}$ and $\mathbf{m}$ are as in Construction 3.2.1.

(i) For technical reasons, we only assumed in the construction that $\sigma_{i}$ has period $m_{i}$. However, we will see below that a necessary condition for $\operatorname{LT}_{\mathbf{m}}(\mathfrak{s}, \boldsymbol{\sigma}, \mathfrak{h})$ to be a Lie torus is that $\left|\sigma_{i}\right|=m_{i}$ for all $i$, at which point we will simplify notation by deleting the subscript $\mathbf{m}$.

(ii) One choice of $\mathfrak{h}$ satisfying the requirements in the construction is a Cartan subalgebra of $\mathfrak{s}^{\sigma}$ [J] Thms. III.10 and III.17]. Conversely, we will see below that a necessary condition for $\operatorname{LT}_{\mathbf{m}}(\mathfrak{s}, \boldsymbol{\sigma}, \mathfrak{h})$ to be a Lie torus is that $\mathfrak{h}$ is a Cartan subalgebra of $\mathfrak{s}^{\sigma}$. Again, for technical reasons we do not make that assumption at the outset.

(iii) $\operatorname{LT}_{\mathbf{m}}(\mathfrak{s}, \boldsymbol{\sigma}, \mathfrak{h})$ is fgc and centreless. (In fact it is fgc and $\mathbb{Z}^{n}$-graded-centralsimple by Theorem 3.1.4.)

In order to describe conditions for $\operatorname{LT}_{\mathbf{m}}(\mathfrak{s}, \boldsymbol{\sigma}, \mathfrak{h})$ to be a Lie torus, it will be convenient to introduce the following terminology for modules. 
Definition 3.2.3. If $\mathfrak{g}$ is a finite dimensional simple Lie algebra, $\mathfrak{h}$ is a Cartan subalgebra of $\mathfrak{g}$ and $V$ is a finite dimensional module for $\mathfrak{g}$, we say that $V$ satisfies condition $(\mathrm{M})$ if

(M) $V$ is irreducible of dimension $>1$ and the weights of $V$ relative to $\mathfrak{h}$ are contained in $\Delta(\mathfrak{g}, \mathfrak{h})_{\text {en }}$, where $\mathfrak{h}$ is a Cartan subalgebra of $\mathfrak{g}$.

(See (1.1.2) for the notation $\left.\Delta(\mathfrak{g}, \mathfrak{h})_{\mathrm{en}}.\right)$ Note that the validity of condition (M) does not depend on the choice of $\mathfrak{h}$.

It is easy to list the modules satisfying this condition:

Lemma 3.2.4. Suppose that $\mathfrak{g}$ is a finite dimensional Lie simple algebra with Cartan subalgebra $\mathfrak{h}$. We fix a base for the root system $\Delta_{\mathfrak{g}}=\Delta(\mathfrak{g}, \mathfrak{h})$. For a dominant integral weight $\mu$ for $\mathfrak{g}$ (relative to $\mathfrak{h}$ and the chosen base), let $V(\mu)$ be the irreducible module with highest weight $\mu$. Let $\theta$ and $\theta_{\mathrm{sh}}$ denote the highest root and the highest short root of $\mathfrak{g}$ respectively.

If $V$ is a finite dimensional $\mathfrak{g}$-module, then $V$ satisfies condition $(M)$ if and only if $V$ is isomorphic to one of the following:

(i) the adjoint module $V(\theta)$,

(ii) the little adjoint module $V\left(\theta_{\mathrm{sh}}\right)$ if $\Delta_{\mathfrak{g}}$ is not simply laced,

(iii) the symmetric module $V\left(2 \theta_{\mathrm{sh}}\right)$ if $\Delta_{\mathfrak{g}}$ is of type $B_{\ell}$ with $\ell \geq 1$.

Moreover, if $V$ satisfies condition $(M)$, then the multiplicity of each nonzero weight in $V$ is 1 . Also, if $V$ and $V^{\prime}$ satisfy condition $(M)$, then $V$ and $V^{\prime}$ have a nonzero weight in common.

Proof. The first statement follows from the fact that the orbits under the Weyl group of $\Delta_{\mathfrak{g}}$ acting on $\left(\Delta_{\mathfrak{g}}\right)_{\text {en }}$ are represented by $0, \theta, \theta_{\mathrm{sh}}$ (if $\Delta_{\mathfrak{g}}$ is not simply laced), and $2 \theta_{\mathrm{sh}}$ (if $\Delta_{\mathfrak{g}}$ is of type $\mathrm{B}_{\ell}$ with $\ell \geq 1$ ). The last two statements follow from the first statement and a calculation of the weights and multiplicities of the modules in (i)-(iii) (see for example [ABG, Chapter 2]).

In Construction 3.2.1, if $\mathfrak{g}=\mathfrak{s}^{\sigma}$ and $\bar{\lambda} \in \bar{\Lambda}$, then $\mathfrak{s}^{\bar{\lambda}}$ is a $\mathfrak{g}$-module under the adjoint action.

Proposition 3.2.5. Let $\mathfrak{s}, \boldsymbol{\sigma}, \mathfrak{h}$ and $\mathbf{m}$ be as Construction 3.2 .1 , and define $\Delta$ and $Q$ by (22). Put $\mathfrak{g}=\mathfrak{s}^{\sigma}$ and $\Delta_{\mathfrak{g}}=\Delta(\mathfrak{g}, \mathfrak{h})$. Then, the following statements are equivalent:

(a) $\Delta$ is a finite irreducible root system in $\mathfrak{h}^{*}$ and $\operatorname{LT}_{\mathbf{m}}(\mathfrak{s}, \boldsymbol{\sigma}, \mathfrak{h})$ is a Lie $\mathbb{Z}^{n}$-torus of type $\Delta$.

(b) $\mathfrak{h}$ is a Cartan subalgebra of $\mathfrak{g}, m_{i}=\left|\sigma_{i}\right|$ for each $i$, and $\boldsymbol{\sigma}$ satisfies the following conditions:

(A1) $\mathfrak{g}$ is a simple Lie algebra.

(A2) If $0 \neq \bar{\lambda} \in \operatorname{supp}_{\bar{\Lambda}}(\mathfrak{s})$, then, $\mathfrak{s}^{\bar{\lambda}} \simeq U^{\bar{\lambda}} \oplus V^{\bar{\lambda}}$ as a $\mathfrak{g}$-module, where $\mathfrak{g}$ acts trivially on $U^{\bar{\lambda}}$ and either $V^{\bar{\lambda}}=0$ or $V^{\bar{\lambda}}$ satisfies condition (M).

(A3) $\left|\left\langle\sigma_{1}, \ldots, \sigma_{n}\right\rangle\right|=\prod_{i=1}^{n}\left|\sigma_{i}\right|$.

Moreover, if (a) and (b) hold, then $\operatorname{LT}_{\mathbf{m}}(\mathfrak{s}, \boldsymbol{\sigma}, \mathfrak{h})$ satisfies (LT5), $(\mathfrak{g} \otimes 1, \mathfrak{h} \otimes 1)$ is the root-grading pair for $\operatorname{LT}_{\mathbf{m}}(\mathfrak{s}, \boldsymbol{\sigma}, \mathfrak{h})$, and

$$
\Delta_{\text {ind }}=\Delta_{\mathfrak{g}}
$$

In fact, in that case,

$$
\Delta=\Delta_{\mathfrak{g}} \quad \text { or } \quad \Delta=\left(\Delta_{\mathfrak{g}}\right)_{\mathrm{en}}
$$


and $\Delta \neq \Delta_{\mathfrak{g}}$ if and only if $\mathfrak{g}$ has type $B_{\ell}$ for some $\ell \geq 1$ and $V^{\bar{\lambda}}$ is isomorphic to the symmetric module for some $0 \neq \bar{\lambda} \in \operatorname{supp}_{\bar{\Lambda}}(\mathfrak{s})$.

Proof. Let $\mathcal{L}=\operatorname{LT}_{\mathbf{m}}(\mathfrak{s}, \boldsymbol{\sigma}, \mathfrak{h})$.

"(b) $\Rightarrow(\mathrm{a})$ " Assume that (b) holds. Now $\Delta$ is the union of the sets of weights of the $\mathfrak{g}$-modules $\mathfrak{s}^{\bar{\lambda}}, \bar{\lambda} \in \operatorname{supp}_{\bar{\Lambda}}(\mathfrak{s})$. So it follows from (A1), (A2) and Lemma 3.2.4 that we have the last statement of the proposition. It then follows from (25) that (24) holds.

We claim next that $\mathfrak{s}$ is a Lie $\bar{\Lambda}$-torus of type $\Delta$ satisfying (LT5). So we must check (LT1)-(LT5) for $\mathfrak{s}$. First since $\Delta=\Delta(\mathfrak{s}, \mathfrak{h})$ and since $\Delta_{\text {ind }}=\Delta_{\mathfrak{g}},(\mathrm{LT} 1)$ and (LT2)(i) are clear. Thus, since $\mathfrak{s}$ is simple, (LT3) also holds for $\mathfrak{s}$ by Remark 1.1.8, Next, by assumption (A3) and Lemma 3.1.3, we have $\left\langle\operatorname{supp}_{\mathbb{Z}^{n}}\left(M_{\mathbf{m}}(\mathfrak{s}, \boldsymbol{\sigma})\right)\right\rangle=\mathbb{Z}^{n}$, so, by (21), $\left\langle\operatorname{supp}_{\bar{\Lambda}}(\mathfrak{s})\right\rangle=\bar{\Lambda}$. Thus, (LT4) holds for $\mathfrak{s}$. Also, again since $\Delta=\Delta(\mathfrak{s}, \mathfrak{h})$, (LT5) holds for $\mathfrak{s}$. So we are left only with (LT2)(ii) for $\mathfrak{s}$. For this, note first that by (A2) and Lemma 3.2.4 if $\alpha \in \Delta^{\times}$and $0 \neq \bar{\lambda} \in \operatorname{supp}_{\bar{\Lambda}}(\mathfrak{s})$, the multiplicity of the $\alpha$-weight space in the module $\mathfrak{s}^{\bar{\lambda}}$ is $\leq 1$. But this is also true if $\bar{\lambda}=\overline{0}$. So

$$
\operatorname{dim}\left(\mathfrak{s}_{\alpha}^{\bar{\lambda}}\right) \leq 1
$$

for $\alpha \in \Delta^{\times}, \bar{\lambda} \in \bar{\Lambda}$. Next let $(,)_{\mathfrak{s}}$ be the Killing form on $\mathfrak{s}$. Then $(,)_{\mathfrak{s}}$ is a nondegenerate invariant symmetric bilinear form on $\mathfrak{s}$. Furthermore, the automorphisms $\sigma_{1}, \ldots, \sigma_{n}$ preserve this form and $\operatorname{ad}_{\mathfrak{s}}(h)$ is a skew transformation relative to this form for $h \in \mathfrak{h}$. Hence it follows that

$$
\left(\mathfrak{s}_{\alpha}^{\bar{\lambda}}, \mathfrak{s}_{\beta}^{\bar{\mu}}\right)_{\mathfrak{s}}=0 \quad \text { unless } \alpha+\beta=0 \text { and } \bar{\lambda}+\bar{\mu}=0
$$

for $\alpha, \beta \in Q$ and $\bar{\lambda}, \bar{\mu} \in \bar{\Lambda}$. Now let $(\alpha, \bar{\lambda}) \in \operatorname{supp}_{Q \times \bar{\Lambda}}(\mathfrak{s})$ with $\alpha \in \Delta^{\times}$. Then, by (27) and the nondegeneracy of $(,)_{\mathfrak{s}}$, we have $(-\alpha,-\bar{\lambda}) \in \operatorname{supp}_{Q \times \bar{\Lambda}}(\mathfrak{s})$. So we can choose $0 \neq e_{\alpha}^{\bar{\lambda}} \in \mathfrak{s}_{\alpha}^{\bar{\lambda}}$ and $0 \neq f_{\alpha}^{\bar{\lambda}} \in \mathfrak{s}_{-\alpha}^{-\bar{\lambda}}$, in which case we have $\mathfrak{s}_{\alpha}^{\bar{\lambda}}=k e_{\alpha}^{\bar{\lambda}}$ and $\mathfrak{s}_{\alpha}^{\bar{\lambda}}=k f_{\alpha}^{\bar{\lambda}}$ by (26). Next we set $h_{\alpha}^{\bar{\lambda}}=\left[e_{\alpha}^{\bar{\lambda}}, f_{\alpha}^{\bar{\lambda}}\right] \in \mathfrak{s}_{0}^{\overline{0}}=\mathfrak{h}$. Then, if $h \in \mathfrak{h}$, we have

$$
\left(h, h_{\alpha}^{\bar{\lambda}}\right)_{\mathfrak{s}}=\left(h,\left[e_{\alpha}^{\bar{\lambda}}, f_{\alpha}^{\bar{\lambda}}\right]\right)_{\mathfrak{s}}=\left(\left[h, e_{\alpha}^{\bar{\lambda}}\right], f_{\alpha}^{\bar{\lambda}}\right)_{\mathfrak{s}}=\alpha(h)\left(e_{\alpha}^{\bar{\lambda}}, f_{\alpha}^{\bar{\lambda}}\right)_{\mathfrak{s}}
$$

Thus, $h_{\alpha}^{\bar{\lambda}}$ is a nonzero multiple of $\alpha^{\vee}$, so, adjusting $f_{\alpha}^{\bar{\lambda}}$ by a nonzero scalar, we can assume that $h_{\alpha}^{\bar{\lambda}}=\alpha^{\vee}$. Therefore we have (LT2)(ii) for $\mathfrak{s}$. Thus, as claimed, $\mathfrak{s}$ is a Lie $\bar{\Lambda}$-torus of type $\Delta$ satisfying (LT5).

To see that $\mathcal{L}$ is a $\mathbb{Z}^{n}$-Lie torus of type $\Delta$ that also satisfies (LT5), we must check the axioms (LT1)-(LT5) for $\mathcal{L}$. Indeed, by (23), if $(\alpha, \lambda) \in Q \times \mathbb{Z}^{n}$, we have

$$
(\alpha, \lambda) \in \operatorname{supp}_{Q \times \mathbb{Z}^{n}}(\mathcal{L}) \Longleftrightarrow(\alpha, \bar{\lambda}) \in \operatorname{supp}_{Q \times \bar{\Lambda}}(\mathfrak{s}) .
$$

Using this fact, the axioms (LT1), (LT2), (LT4) and (LT5) for $\mathcal{L}$ follow easily from the corresponding axioms for $\mathfrak{s}$. Finally, since $\mathcal{L}$ is a graded-simple $\mathbb{Z}^{n}$-graded algebra by Theorem 3.1.4, $\mathcal{L}$ satisfies (LT3) by Remark 1.1.8. Thus, as claimed, $\mathcal{L}$ is a Lie $\mathbb{Z}^{n}$-torus of type $\Delta$ satisfying (LT5).

So we have established (a) as well as (24) and (25). Also, by Proposition 1.2.2(vi), the root-grading pair for $\mathcal{L}$ is $\left(\mathcal{L}^{0}, \mathcal{L}_{0}^{0}\right)=\left(\mathfrak{s}^{\overline{0}} \otimes 1, \mathfrak{s}_{0}^{0} \otimes 1\right)=(\mathfrak{g} \otimes 1, \mathfrak{h} \otimes 1)$.

"(a) $\Rightarrow$ (b)" Suppose (a) holds. By Proposition 1.2 .2 (vi), the grading pair for $\mathcal{L}$ is $\left(\mathcal{L}^{0}, \mathcal{L}_{0}^{0}\right)=\left(\mathfrak{g} \otimes 1, \mathfrak{s}_{0}^{\overline{0}} \otimes 1\right)$. So, by Proposition 1.2.2, (ii) and (iii), $\mathfrak{g}$ is simple, $\mathfrak{s}_{0}^{\overline{0}}$ is a Cartan subalgebra of $\mathfrak{g}$, and $\operatorname{dim}\left(\mathfrak{s}_{0}^{\overline{0}}\right)=\operatorname{rank}(Q)$. But, since $\Delta$ is a root system in $\mathfrak{h}^{*}$, we have $\operatorname{dim}(\mathfrak{h})=\operatorname{rank}(Q)$. Hence, since $\mathfrak{s}_{0}^{\overline{0}} \supseteq \mathfrak{h}$, we have $\mathfrak{s}_{0}^{\overline{0}}=\mathfrak{h}$. Thus, $\mathfrak{g}$ is simple and $\mathfrak{h}$ is a Cartan subalgebra of $\mathfrak{g}$. 
By (LT4), $\mathbb{Z}^{n}=\left\langle\operatorname{supp}_{\mathbb{Z}^{n}}(\mathcal{L})\right\rangle$, so, by Lemma 3.1.3. $\left|\left\langle\sigma_{1}, \ldots, \sigma_{n}\right\rangle\right|=m_{1} \cdots m_{n}$. Thus we have $m_{i}=\left|\sigma_{i}\right|$, and (A1) and (A3) hold.

Next, if $\alpha \in \Delta_{\text {ind }}^{\times}$, then, by (LT2)(i), we have $0 \neq \mathcal{L}_{\alpha}^{0}=\mathfrak{s}_{\alpha}^{\overline{0}} \otimes 1=\mathfrak{g}_{\alpha} \otimes 1$, so $\alpha \in \Delta_{\mathfrak{g}}$. Therefore, $\Delta_{\text {ind }} \subseteq \Delta_{\mathfrak{g}}$, so $\Delta_{\text {ind }} \subseteq \Delta_{\mathfrak{g}} \subseteq \Delta$. Since $\Delta_{\mathfrak{g}}$ is reduced, we have $\Delta_{\text {ind }}=\Delta_{\mathfrak{g}}$. So $\Delta=\Delta_{\mathfrak{g}}$ or $\Delta=\left(\Delta_{\mathfrak{g}}\right)_{\mathrm{en}}$.

Now to prove (A2) let $0 \neq \bar{\lambda} \in \operatorname{supp}_{\bar{\Lambda}}(\mathfrak{s})$. Since $\Delta \subseteq\left(\Delta_{\mathfrak{g}}\right)_{\mathrm{en}}$, the weights of the $\mathfrak{g}$-module $\mathfrak{s}^{\bar{\lambda}}$ are contained in $\left(\Delta_{\mathfrak{g}}\right)_{\text {en }}$. It therefore remains to show that this $\mathfrak{g}$-module has at most one irreducible component of dimension $\geq 2$. Suppose the contrary. Then, by Lemma 3.2.4, the $\alpha$-weight space in $\mathfrak{s}^{\bar{\lambda}}$ has dimension $>1$ for some $\alpha \in \Delta^{\times}$. So $\operatorname{dim} \mathfrak{s}_{\alpha}^{\bar{\lambda}} \geq 2$ and hence (by (23) $) \operatorname{dim} \mathcal{L}_{\alpha}^{\lambda} \geq 2$, contradicting (LT2)(ii).

Definition 3.2.6. Suppose that $\mathfrak{s}$ is a finite dimensional simple Lie algebra over $k, \boldsymbol{\sigma}=\left(\sigma_{1}, \ldots, \sigma_{n}\right)$ is a sequence in $\operatorname{cfo}_{n}(\mathfrak{s})$ satisfying (A1)-(A3), and $\mathfrak{h}$ is a Cartan subalgebra of $\mathfrak{g}=\mathfrak{s}^{\boldsymbol{\sigma}}$. Let $\mathbf{m}=\left(m_{1}, \ldots, m_{n}\right)$, where $m_{i}=\left|\sigma_{i}\right|, \Delta=\Delta(\mathfrak{s}, \mathfrak{h})$ and $Q=\operatorname{span}_{\mathbb{Z}}(\Delta)$. Since $\mathbf{m}$ is determined by $\boldsymbol{\sigma}$, we simplify notation and write

$$
\operatorname{LT}(\mathfrak{s}, \boldsymbol{\sigma}, \mathfrak{h}):=\operatorname{LT}_{\mathbf{m}}(\mathfrak{s}, \boldsymbol{\sigma}, \mathfrak{h})
$$

Then, by Proposition 3.2.5 and Remark 3.2.2(iii), LT $(\mathfrak{s}, \boldsymbol{\sigma}, \mathfrak{h})$ is an fgc centreless $\mathbb{Z}^{n}$-Lie torus of type $\Delta$. We call $\operatorname{LT}(\mathfrak{s}, \boldsymbol{\sigma}, \mathfrak{h})$ the multiloop Lie $\mathbb{Z}^{n}$-torus (or simply the multiloop Lie torus) determined by $\mathfrak{s}, \boldsymbol{\sigma}$ and $\mathfrak{h}$.

Remark 3.2.7. Suppose that we have the assumptions and notation of Definition 3.2.6. Suppose that $\mathfrak{h}^{\prime}$ is another Cartan subalgebra of $\mathfrak{g}$. Note that $\operatorname{LT}(\mathfrak{s}, \boldsymbol{\sigma}, \mathfrak{h})$ and $\operatorname{LT}\left(\mathfrak{s}, \boldsymbol{\sigma}, \mathfrak{h}^{\prime}\right)$ are equal as $\mathbb{Z}^{n}$-graded algebras. Hence, by Proposition 2.1.3. $\operatorname{LT}(\mathfrak{s}, \boldsymbol{\sigma}, \mathfrak{h})$ and $\operatorname{LT}\left(\mathfrak{s}, \boldsymbol{\sigma}, \mathfrak{h}^{\prime}\right)$ are bi-isomorphic as Lie tori. Thus, up to bi-isomorphism, the Lie torus $\operatorname{LT}(\mathfrak{s}, \boldsymbol{\sigma}, \mathfrak{h})$ does not depend on the choice of the Cartan subalgebra $\mathfrak{h}$ of $\mathfrak{g}$.

Example 3.2.8. Suppose that $\mathfrak{s}$ is a finite dimensional simple Lie algebra, $\mathbf{1}=$ $(1, \ldots, 1)$, and $\mathfrak{h}$ is a Cartan subalgebra of $\mathfrak{s}$. Then, $\operatorname{LT}(\mathfrak{s}, \mathbf{1}, \mathfrak{h})=\mathfrak{s} \otimes k\left[z_{1}^{ \pm 1}, \ldots, z_{n}^{ \pm 1}\right]$ is a multiloop Lie torus called the untwisted multiloop Lie $\mathbb{Z}^{n}$-torus determined by $\mathfrak{s}$ and $\mathfrak{h}$.

3.3. The realization theorem. We can now prove our first main result.

Theorem 3.3.1 (Realization Theorem for Lie tori). Suppose that $k$ is an algebraically closed field of characteristic 0 , and $n \geq 1$. Then a centreless Lie $\Lambda$-torus $\mathcal{L}$ of nullity $n$ is bi-isomorphic to a multiloop Lie $\mathbb{Z}^{n}$-torus (as defined in Definition 3.2.6) if and only if $\mathcal{L}$ is fgc.

Proof. The necessity of the fgc condition is noted in Remark 3.2 .2 and so we need only prove the sufficiency. Let $\Lambda$ be a free abelian group of rank $n$ and let $\mathcal{L}$ be an fgc centreless Lie $\Lambda$-torus of type $\Delta$. We let $Q=\operatorname{span}_{\mathbb{Z}}(\Delta)$, so $\mathcal{L}$ is a $Q \times \Lambda$-graded algebra. Replacing $\Delta$ by $\Delta_{\text {ind }}$ if necessary we can assume (see Remark 1.1.11) that $\mathcal{L}$ satisfies (LT5). That is,

$$
\Delta=\operatorname{supp}_{Q}(\mathcal{L})
$$

Now, by Proposition 1.4.2 $\Lambda / \Gamma_{\Lambda}(\mathcal{L})$ is finite. Also by Proposition 1.4.1(i), $\mathcal{L}$ is a graded-central-simple $\Lambda$-graded algebra. Therefore by Theorem 3.1.4 the $\Lambda$ graded algebra $\mathcal{L}$ is isograded-isomorphic to the $\mathbb{Z}^{n}$-graded algebra $M_{\mathbf{m}}(\mathfrak{s}, \boldsymbol{\sigma})$ for 
some finite dimensional simple Lie algebra $\mathfrak{s}$, some $\boldsymbol{\sigma} \in \operatorname{cfo}_{n}(\mathfrak{s})$ and some $\mathbf{m} \in \mathbb{Z}_{+}^{n}$ with $\sigma^{\mathrm{m}}=\mathbf{1}$.

So we have an algebra isomorphism $\varphi: \mathcal{L} \rightarrow M_{\mathbf{m}}(\mathfrak{s}, \boldsymbol{\sigma})$ and a group isomorphism $\nu: \Lambda \rightarrow \mathbb{Z}^{n}$ such that $\varphi\left(\mathcal{L}^{\lambda}\right)=M_{\mathbf{m}}(\mathfrak{s}, \boldsymbol{\sigma})^{\nu(\lambda)}$ for $\lambda \in \Lambda$. We use $\varphi$ to transfer the $Q$-graded structure of $\mathcal{L}$ to $M_{\mathbf{m}}(\mathfrak{s}, \boldsymbol{\sigma})$. Then $M_{\mathbf{m}}(\mathfrak{s}, \boldsymbol{\sigma})$ becomes a Lie $\Lambda$-torus of type $\Delta$. We now use $\nu$ to identify $\Lambda$ with $\mathbb{Z}^{n}$ and we use $\varphi$ to identify

$$
\mathcal{L}=M_{\mathbf{m}}(\mathfrak{s}, \boldsymbol{\sigma})
$$

as a $Q \times \Lambda$-graded algebra.

Next let $(\mathfrak{g}, \mathfrak{h})$ be the root-grading pair for $\mathcal{L}$. Then, by Proposition 1.2 .2 (vi), $\mathfrak{g}=\mathcal{L}^{0}=\mathfrak{s}^{\overline{0}} \otimes 1$, so we can identify $\mathfrak{g}$ with the subalgebra $\mathfrak{s}_{\overline{0}}$ of $\mathfrak{s}$ (by identifying $x \otimes 1$ with $x$ ).

Now we use the linear isomorphism ${ }^{\sim}$ in Proposition 1.2.2(iii) to identify $\Delta$ with a finite irreducible root system in $\mathfrak{h}^{*}$ such that $\Delta_{\text {ind }}=\Delta(\mathfrak{g}, \mathfrak{h})$ and

$$
\mathcal{L}_{\alpha}=\{x \in \mathcal{L} \mid[h, x]=\alpha(h) x \text { for } h \in \mathfrak{h}\}
$$

for $\alpha \in Q$. Thus, by (28), $\Delta=\Delta(\mathcal{L}, \mathfrak{h})=\Delta(\mathfrak{s}, \mathfrak{h})$. Our result now follows from Proposition $3.2 .5((\mathrm{a}) \Rightarrow(\mathrm{b}))$.

3.4. The case $n=1$. In this subsection, we show that when $n=1$, conditions (A1) and (A2) are equivalent to the condition that $\sigma$ is a diagram automorphism. ((A3) is automatic when $n=1$.) So, when $n=1$, Theorem 3.3.1 coincides with the classical realization theorem for affine Kac-Moody Lie algebras (see Remark 3.4.2 below).

We first recall the notion of a diagram automorphism of a finite dimensional simple Lie algebra $\mathfrak{s}$. If $\mathfrak{t}$ is a Cartan subalgebra of $\mathfrak{s},\left\{\alpha_{i}\right\}_{i=1}^{r}$ is a base for $\Delta(\mathfrak{s}, \mathfrak{t})$, and $0 \neq e_{i} \in \mathfrak{s}_{\alpha_{i}}$ for $1 \leq i \leq r$, then the pair $\left(\left\{\alpha_{i}\right\},\left\{e_{i}\right\}\right)$ is called an épinglage of $(\mathfrak{s}, \mathfrak{t})$ B2, Chap. VIII, $\left.\S 4, \mathrm{n}^{\circ} 1\right]$. A diagram automorphism of $\mathfrak{s}$ is an automorphism $\sigma$ of $\mathfrak{s}$ that stabilizes $\mathfrak{t}$ and $\left\{e_{i}\right\}$ for some choice of Cartan subalgebra $\mathfrak{t}$ and épinglage $\left(\left\{\alpha_{i}\right\},\left\{e_{i}\right\}\right)$, in which case $\sigma$ is called a diagram automorphism of $\mathfrak{s}$ relative to $\mathfrak{t}$ and $\left(\left\{\alpha_{i}\right\},\left\{e_{i}\right\}\right)$. Moreover, any diagram automorphism of $\mathfrak{s}$ is conjugate in $\operatorname{Aut}_{k}(\mathfrak{s})$ to one relative to a fixed Cartan subalgebra and épinglage B2, Chap. VIII, $\S 5$, Prop. 5].

Proposition 3.4.1. A finite order automorphism $\sigma$ of a finite dimensional simple Lie algebra $\mathfrak{s}$ satisfies (A1) and (A2) if and only if $\sigma$ is a diagram automorphism.

Proof. First we fix some notation. Let $\left\{e_{\alpha}, h_{i}\right\}=\left\{e_{\alpha}: \alpha \in \Delta(\mathfrak{s}, \mathfrak{t})^{\times}\right\} \cup\left\{h_{i}: 1 \leq\right.$ $i \leq r\}$ be a Chevalley basis for $\mathfrak{s}$. Set $\mathfrak{t}=\sum k h_{i}$, a Cartan subalgebra of $\mathfrak{s}$. Let $\left\{\alpha_{i}\right\}_{i=1}^{r}$ be a base for $\Delta(\mathfrak{s}, \mathfrak{t})$, in which case $\left(\left\{\alpha_{i}\right\},\left\{e_{\alpha_{i}}\right\}\right)$ is an épinglage of $(\mathfrak{s}, \mathfrak{t})$.

Now, by [KW, Proposition 4.29], replacing $\sigma$ by a conjugate in $\operatorname{Aut}_{k}(\mathfrak{s})$, we may assume that

$$
\sigma=\tau \hat{\sigma}
$$

where $\tau, \hat{\sigma} \in \operatorname{Aut}_{k}(\mathfrak{s}), \tau \hat{\sigma}=\hat{\sigma} \tau, \hat{\sigma}$ is a diagram automorphism of $\mathfrak{s}$ relative to $\mathfrak{t}$ and $\left(\left\{\alpha_{i}\right\},\left\{e_{\alpha_{i}}\right\}\right), \tau$ fixes $\mathfrak{t}$ pointwise, and $\tau$ is diagonal relative to the Chevalley basis $\left\{e_{\alpha}, h_{i}\right\}$ of $\mathfrak{s}$. We will show that under these circumstances

$$
\sigma \text { is conjugate to } \hat{\sigma} \text { in } \operatorname{Aut}_{k}(\mathfrak{s}) \Longleftrightarrow \sigma \text { satisfies (A1) and (A2). }
$$

This suffices to prove the proposition.

We begin by reducing the proof of (29) to the case when $k=\mathbb{C}$. (We do this since we wish to use Kac's classification of finite order automorphisms of $\mathfrak{s}$, which has 
been carried out only in that case (see $[\mathrm{K}$, Chap. 8] or [GOV, Ch. 3, §§3.6-3.11]).) For this, let $\overline{\mathbb{Q}}$ be an algebraic closure of the rational field, which we regard as a subfield of both $k$ and $\mathbb{C}$. Let $\mathfrak{s}(\overline{\mathbb{Q}})$ be the $\overline{\mathbb{Q}}$-span of the Chevalley basis $\left\{e_{\alpha}, h_{i}\right\}$ in $\mathfrak{s}$, and let $\mathfrak{s}(k)=\mathfrak{s}(\overline{\mathbb{Q}}) \otimes_{\overline{\mathbb{Q}}} k, \mathfrak{s}(\mathbb{C})=\mathfrak{s}(\overline{\mathbb{Q}}) \otimes_{\overline{\mathbb{Q}}} \mathbb{C}$. We identify $\mathfrak{s}(k)$ with $\mathfrak{s}$, regard $\mathfrak{s}(\overline{\mathbb{Q}})$ as a $\overline{\mathbb{Q}}$-subalgebra of both $\mathfrak{s}$ and $\mathfrak{s}(\mathbb{C})$, and regard $\operatorname{Aut}_{\overline{\mathbb{Q}}}(\mathfrak{s}(\overline{\mathbb{Q}}))$ as a subgroup of both $\operatorname{Aut}_{k}(\mathfrak{s})$ and $\operatorname{Aut}_{\mathbb{C}}(\mathfrak{s}(\mathbb{C}))$. Now $\tau$ has finite order and acts diagonally with respect to the Chevalley basis $\left\{e_{\alpha}, h_{i}\right\}$, so $\tau$ is in $\operatorname{Aut}_{\overline{\mathbb{Q}}}(\mathfrak{s}(\overline{\mathbb{Q}}))$. On the other hand, $\hat{\sigma}$ is certainly in $\operatorname{Aut}_{\overline{\mathbb{Q}}}(\mathfrak{s}(\overline{\mathbb{Q}}))$, and therefore so is $\sigma$. But $\sigma$ and $\hat{\sigma}$ are conjugate in $\operatorname{Aut}_{k}(\mathfrak{s})$ if and only if they are conjugate in $\operatorname{Aut}_{\overline{\mathbb{Q}}}(\mathfrak{s}(\overline{\mathbb{Q}})$ ) (since conjugacy is checked by solving a system of polynomial equations over $\overline{\mathbb{Q}})$, which in turn holds if and only if they are conjugate in $\operatorname{Aut}_{\mathbb{C}}(\mathfrak{s}(\mathbb{C}))$. Also, it is clear that $\sigma$ satisfies $(\mathrm{A} 1)$ and $(\mathrm{A} 2)$ in $\operatorname{Aut}_{k}(\mathfrak{s})$ if and only if $\sigma$ satisfies (A1) and (A2) in $\operatorname{Aut}_{\overline{\mathbb{Q}}}(\mathfrak{s}(\overline{\mathbb{Q}}))$, which in turn holds if and only if $\sigma$ satisfies (A1) and (A2) in $\operatorname{Aut}_{\mathbb{C}}(\mathfrak{s}(\mathbb{C}))$. So we may assume that $k=\mathbb{C}$.

It is well known that any diagram automorphism satisfies (A1) and (A2) $\mathrm{K}$, Props. 7.9 and 7.10]. In fact, for $0 \neq \bar{\lambda} \in \bar{\Lambda}$, the $\mathfrak{g}$-module $\mathfrak{s}^{\bar{\lambda}}$ itself satisfies condition (M). So we have " $\Rightarrow$ " in (29).

For the converse suppose that $\sigma$ satisfies (A1) and (A2). Now, $\tau$ fixes $\mathfrak{t}$ pointwise and hence $\tau$ stabilizes the root spaces in $\mathfrak{s}$ relative to $\mathfrak{t}$. It follows that $\tau$ lies in the connected component of the identity in the algebraic group $\operatorname{Aut}_{k}(\mathfrak{s})$. Thus, replacing $\sigma$ by a conjugate in $\operatorname{Aut}_{k}(\mathfrak{s})$, we may assume that

$$
\tau=\exp (2 \pi i h),
$$

where $h \in \mathfrak{h}^{\hat{\sigma}}$ has barycentric coordinates satisfying certain positivity conditions GOV, Thms. 3.11 and 3.16]. (We will not need the definition of these coordinates nor the precise conditions, but only the consequences that we mention below.) Let $\mathfrak{g}=\mathfrak{s}^{\sigma}$ and $\hat{\mathfrak{g}}=\mathfrak{s}^{\hat{\sigma}}$. By (A1), $\mathfrak{g}$ and $\hat{\mathfrak{g}}$ are simple. Also, since $\tau$ fixes $\mathfrak{t}$ pointwise, we see that $\mathfrak{t}^{\sigma}=\mathfrak{t}^{\hat{\sigma}}$, and we set

$$
\mathfrak{h}:=\mathfrak{t}^{\sigma}=\mathfrak{t}^{\hat{\sigma}} .
$$

Then, since $\mathfrak{h}=\mathfrak{t}^{\hat{\sigma}}$, the centralizer $C_{\mathfrak{s}}(\mathfrak{h})$ of $\mathfrak{h}$ in $\mathfrak{s}$ is a Cartan subalgebra of $\mathfrak{s}[\mathrm{P}$, Theorem 9]. But $\mathfrak{t} \subseteq C_{\mathfrak{s}}(\mathfrak{h})$, so

$$
\mathfrak{t}=C_{\mathfrak{s}}(\mathfrak{h}) .
$$

Thus, any Cartan subalgebra of $\mathfrak{g}$ containing $\mathfrak{h}$ is contained in $\mathfrak{t}$ and hence lies in $\mathfrak{g} \cap \mathfrak{t}=\mathfrak{h}$. So $\mathfrak{h}$ is a Cartan subalgebra of $\mathfrak{g}$, and similarly $\mathfrak{h}$ is a Cartan subalgebra of $\hat{\mathfrak{g}}$.

We now let $\Delta_{\mathfrak{g}}=\Delta(\mathfrak{g}, \mathfrak{h}), \Delta_{\mathfrak{g}}=\Delta(\hat{\mathfrak{g}}, \mathfrak{h})$, and $\Delta=\Delta(\mathfrak{s}, \mathfrak{h})$. Then, by Proposition 3.2.5, $\Delta$ is an irreducible root system in $\mathfrak{h}^{*}, \Delta=\Delta_{\mathfrak{g}}$ or $\Delta=\left(\Delta_{\mathfrak{g}}\right)_{\mathrm{en}}$, and $\Delta=\Delta_{\hat{\mathfrak{g}}}$ or $\Delta=\left(\Delta_{\hat{\mathfrak{g}}}\right)_{\text {en }}$. Hence $\Delta_{\mathfrak{g}}=\Delta_{\hat{\mathfrak{g}}}$, and consequently $\mathfrak{g} \simeq \hat{\mathfrak{g}}$.

Let $m=|\sigma|$ and $\hat{m}=|\hat{\sigma}|$. Then $\left.\hat{\sigma}\right|_{\mathfrak{h}}=\left.\sigma\right|_{\mathfrak{h}}$ and $\hat{m}$ is the order of $\left.\hat{\sigma}\right|_{\mathfrak{h}}$. Thus, $\hat{m}$ divides $m$. In fact, we claim that

$$
\hat{m}=m \text {. }
$$

To see this, it is enough to show that $\tau^{\hat{m}}=1$, or equivalently that $\left.\tau^{\hat{m}}\right|_{\mathfrak{s}_{\alpha}}=1$ for $\alpha \in \Delta$. Hence, since $\Delta \subseteq\left(\Delta_{\mathfrak{g}}\right)_{\text {en }}$, it suffices to show that $\left.\tau^{\hat{m}}\right|_{\mathfrak{s}_{\alpha}}=1$ for $\alpha \in\left(\Delta_{\mathfrak{g}}\right)_{\text {en }}$. So, since $\tau=\exp (2 \pi i h)$, it is enough to show that $\left.\tau^{\hat{m}}\right|_{\mathfrak{g}_{\alpha}}=1$ for $\alpha \in \Delta_{\mathfrak{g}}$. But if $\alpha \in \Delta_{\mathfrak{g}}$ and $x \in \mathfrak{g}_{\alpha}, \tau^{\hat{m}}(x)=\sigma^{\hat{m}}(x)=x$, proving the claim.

If $\hat{m}=m=1$, we have $\sigma=1$. So we can assume that $\hat{m}=m=2$ or 3 . 
Now let $X_{r}$ be the type of the simple Lie algebra $\mathfrak{s}$, let $\tilde{\Pi}^{(\hat{\sigma})}=\left\{\beta_{0}, \beta_{1}, \ldots, \beta_{N}\right\}$ be the set of nodes of an affine Dynkin diagram of type $X_{n}^{(\hat{m})}$, and let $n_{0}^{\prime}, \ldots, n_{N}^{\prime}$ be the standard labels on the diagram $X_{n}^{(\hat{m})}$ as in [GOV, Table 3, p. 228]. (These are the same as the labels in [K, Tables Aff 2 and 3] except for type $A_{2 \ell}^{(2)}$ where the labels are reversed.) Then, we can identify the sub-diagram $\left\{\beta_{1}, \ldots, \beta_{N}\right\}$ with the Dynkin diagram of $\Delta_{\mathfrak{g}}$ (relative to some base) [GOV, Ch. 3, §3.9]. With this notation, the barycentric coordinates $x_{0}, \ldots, x_{N}$ of $h$ satisfy $x_{i}=\frac{p_{i}}{m}$, where $p_{0}, \ldots, p_{N}$ are nonnegative integers satisfying $\sum_{i=0}^{N} n_{i}^{\prime} p_{i}=\frac{m}{\hat{m}}$ GOV, Ch. 3, §3.11].

Since $m=\hat{m}$, it follows that exactly one $p_{i}$, say $p_{a}$, is nonzero. Further, $p_{a}=1$ and

$$
n_{a}^{\prime}=1
$$

Also, since $\mathfrak{s}^{\sigma} \simeq \mathfrak{s}^{\hat{\sigma}}$,

$$
\text { the diagrams } \tilde{\Pi}^{(\hat{\sigma})} \backslash\left\{\beta_{a}\right\} \text { and } \tilde{\Pi}^{(\hat{\sigma})} \backslash\left\{\beta_{0}\right\} \text { are isomorphic }
$$

[ibid]. An examination of the Dynkin diagrams in GOV, Table 3, p. 228] demonstrates that conditions (30) and (31) imply that $\beta_{a}$ is conjugate to $\beta_{0}$ under $\operatorname{Aut}\left(\tilde{\Pi}^{(\hat{\sigma})}\right)$. This together with $p_{a}=1$ implies that $\sigma$ is conjugate to $\hat{\sigma}$ GOV, Ch. $3, \S 3.11]$.

Remark 3.4.2. If $\mathcal{E}$ is an affine Kac-Moody Lie algebra, it is known that the quotient $\mathcal{E}^{(1)} / Z\left(\mathcal{E}^{(1)}\right)$ has the structure of a centreless Lie $\mathbb{Z}$-torus, where $\mathcal{E}^{(1)}$ is the derived algebra of $\mathcal{E}$. Moreover, this Lie torus is always fgc. Hence, by Theorem 3.3 .1 and Proposition 3.4.1, $\mathcal{E}^{(1)} / Z\left(\mathcal{E}^{(1)}\right)$ is bi-isomorphic to a (multi)loop Lie torus $\operatorname{LT}(\mathfrak{s}, \sigma, \mathfrak{h})$, where $\mathfrak{s}$ is a finite dimensional simple Lie algebra, $\sigma$ is a diagram automorphism of $\mathfrak{s}$, and $\mathfrak{h}$ is a Cartan subalgebra of $\mathfrak{s}^{\sigma}$. This is the classical realization theorem [K] Theorems 7.4 and 8.3] for affine Kac-Moody Lie algebras. However, we have not given a new proof of the classical theorem since the fact that $\mathcal{E}^{(1)} / Z\left(\mathcal{E}^{(1)}\right)$ is fgc does not seem clear without using that same theorem.

\section{BI-ISOMORPHISM AND ISOTOPY OF MULTILOOP LiE TORI}

In this section, we give necessary and sufficient conditions for two multiloop Lie tori $\mathcal{L}$ and $\mathcal{L}^{\prime}$ to be bi-isomorphic. We then use that result to give necessary and sufficient conditions for $\mathcal{L}$ and $\mathcal{L}^{\prime}$ to be isotopic.

\subsection{Bi-isomorphism of multiloop Lie tori.}

Theorem 4.1.1. Suppose that $k$ is an algebraically closed field of characteristic 0 and that $n$ is a positive integer. Let $\mathcal{L}=\operatorname{LT}(\mathfrak{s}, \boldsymbol{\sigma}, \mathfrak{h})$ and $\mathcal{L}^{\prime}=\operatorname{LT}\left(\mathfrak{s}^{\prime}, \boldsymbol{\sigma}^{\prime}, \mathfrak{h}^{\prime}\right)$ be multiloop Lie $\mathbb{Z}^{n}$-tori. Then $\mathcal{L}$ and $\mathcal{L}^{\prime}$ are bi-isomorphic if and only if there exists a matrix $P=\left(p_{i j}\right) \in \mathrm{GL}_{n}(\mathbb{Z})$ and an algebra isomorphism $\varphi: \mathfrak{s} \rightarrow \mathfrak{s}^{\prime}$ such that

$$
\boldsymbol{\sigma}^{\prime}=\varphi \boldsymbol{\sigma}^{P} \varphi^{-1} .
$$

Moreover, in that case, $\varphi$ can be chosen with the additional property that $\varphi(\mathfrak{h})=\mathfrak{h}^{\prime}$.

Proof. The first statement follows from Proposition 2.1 .3 and Theorem 3.1.5. For the second statement, suppose that we have $P$ and $\varphi$ as in the first statement. Let $\mathfrak{g}=\mathfrak{s}^{\sigma}$ and $\mathfrak{g}^{\prime}=\mathfrak{s}^{\boldsymbol{\sigma}^{\prime}}$. Then $\varphi(\mathfrak{g})=\mathfrak{g}^{\prime}$. Thus, $\varphi(\mathfrak{h})$ and $\mathfrak{h}^{\prime}$ are Cartan subalgebras of $\mathfrak{g}^{\prime}$. But then there exists an automorphism $\psi_{\mathfrak{g}^{\prime}} \in \operatorname{Aut}_{k}\left(\mathfrak{g}^{\prime}\right)$ of the form $\psi_{\mathfrak{g}^{\prime}}=$ $\exp \left(\operatorname{ad}_{\mathfrak{g}^{\prime}}\left(x_{1}\right)\right) \ldots \exp \left(\operatorname{ad}_{\mathfrak{g}^{\prime}}\left(x_{\ell}\right)\right)$, with $x_{1}, \ldots, x_{\ell}$ ad-nilpotent elements of $\mathfrak{g}^{\prime}$, such 
that $\psi_{\mathfrak{g}^{\prime}}(\phi(\mathfrak{h}))=\mathfrak{h}^{\prime}$ (see the proof of Proposition 2.1.3). Now $\psi_{\mathfrak{g}^{\prime}}$ extends to an automorphism of $\mathfrak{s}^{\prime}$ that commutes with the automorphisms $\sigma_{1}^{\prime}, \ldots, \sigma_{n}^{\prime}$. Replacing $\varphi$ by $\psi \circ \varphi$ we may assume that $\varphi(\mathfrak{h})=\mathfrak{h}^{\prime}$.

Remark 4.1.2. Suppose that $\operatorname{LT}(\mathfrak{s}, \boldsymbol{\sigma}, \mathfrak{h})$ is a multiloop Lie $\mathbb{Z}^{n}$-torus (so $\boldsymbol{\sigma} \in \operatorname{cfo}_{n}(\mathfrak{s})$ satisfies (A1), (A2) and (A3)), and let $P \in \mathrm{GL}_{n}(\mathbb{Z})$. Then, it is easy to see that $\boldsymbol{\sigma}^{P}$ also satisfies (A1) and (A2). However, the reader is cautioned that it is not true in general that $\boldsymbol{\sigma}^{P}$ satisfies (A3). (An example is easily found when $n=2,\left|\sigma_{1}\right|=2$ and $\left|\sigma_{2}\right|=1$.) In fact, any orbit of $\mathrm{GL}_{n}(\mathbb{Z})$ acting on $\operatorname{cfo}_{n}(\mathfrak{s})$ contains an element $\boldsymbol{\sigma}$ that satisfies (A3) (see Proposition 5.1.3 below). This latter fact will be important in our discussion of classification in section 5 (see Theorem 5.3.2(iii) below).

Remark 4.1.3. Suppose that $n=1$. Since any diagram automorphism of $\mathfrak{s}$ is conjugate to its inverse in $\operatorname{Aut}_{k}(\mathfrak{s})$, it follows from Theorem 4.1.1 and Proposition 3.4.1 that two (multi)loop Lie $\mathbb{Z}$-tori $\operatorname{LT}(\mathfrak{s}, \sigma, \mathfrak{h})$ and $\operatorname{LT}\left(\mathfrak{s}^{\prime}, \sigma^{\prime}, \mathfrak{h}^{\prime}\right)$ are bi-isomorphic if and only if there exists an algebra isomorphism $\varphi: \mathfrak{s} \rightarrow \mathfrak{s}^{\prime}$ such that $\sigma^{\prime}=\varphi \sigma \varphi^{-1}$.

Corollary 4.1.4. Suppose that $\mathcal{L}$ and $\mathcal{L}^{\prime}$ are as in Theorem 4.1.1, If $\mathcal{L}$ and $\mathcal{L}^{\prime}$ are bi-isomorphic, then there exists an algebra isomorphism $\varphi: \mathfrak{s} \rightarrow \mathfrak{s}^{\prime}$ such that $\left\langle\boldsymbol{\sigma}^{\prime}\right\rangle=\varphi\langle\boldsymbol{\sigma}\rangle \varphi^{-1}$.

Proof. This follows from Theorem 4.1.1 and (20).

Corollary 4.1.5. Two untwisted multiloop Lie $\mathbb{Z}^{n}$-tori $\operatorname{LT}(\mathfrak{s}, \mathbf{1}, \mathfrak{h})$ and $\operatorname{LT}\left(\mathfrak{s}^{\prime}, \mathbf{1}, \mathfrak{h}^{\prime}\right)$ are bi-isomorphic if and only if $\mathfrak{s}$ and $\mathfrak{s}^{\prime}$ are isomorphic.

Corollary 4.1.6. Suppose that $\mathcal{L}=L T(\mathfrak{s}, \boldsymbol{\sigma}, \mathfrak{h})$ is a multiloop Lie $\mathbb{Z}^{n}$-torus. Then the following are equivalent:

(a) $\sigma=1$.

(b) $\mathcal{L}$ equals the untwisted multiloop Lie $\mathbb{Z}^{n}$-torus $\mathcal{L}=L T(\mathfrak{s}, \mathbf{1}, \mathfrak{h})$.

(c) $\mathcal{L}$ is bi-isomorphic to an untwisted multiloop Lie $\mathbb{Z}^{n}$-torus $L T\left(\mathfrak{s}^{\prime}, \mathbf{1}, \mathfrak{h}^{\prime}\right)$.

(d) $\sigma_{1}, \ldots, \sigma_{n}$ are contained in a common torus of the algebraic group $\operatorname{Aut}_{k}(\mathfrak{s})$ (using the terminology of $[\mathrm{H}, \S 16.2]$ for example).

(e) $\mathfrak{h}$ is a Cartan subalgebra of $\mathfrak{s}$.

Proof. We use the notation $\mathfrak{g}, \Delta_{\mathfrak{g}}, \Delta$ and $Q$ from Proposition 3.2.5. Now "(a) $\Rightarrow$ (b)" and " $(\mathrm{b}) \Rightarrow(\mathrm{c})$ " are trivial. Also, "(c) $\Rightarrow(\mathrm{a})$ " follows from Theorem 4.1.1, hence "(c) $\Rightarrow(\mathrm{d})$ ". To prove "(d) $\Rightarrow(\mathrm{e})$ ", suppose that $\sigma_{1}, \ldots, \sigma_{n}$ are contained in a torus $T$ of $\operatorname{Aut}_{k}(\mathfrak{s})$. Let Lie(T) be the Lie algebra of $T$ in $\operatorname{Lie}\left(\operatorname{Aut}_{k}(\mathfrak{s})\right)=\operatorname{ad}_{\mathfrak{s}}(\mathfrak{s})$. Then $\operatorname{Lie}(T)=\operatorname{ad}_{\mathfrak{s}}(\mathfrak{t})$, where $\mathfrak{t}$ is a Cartan subalgebra of $\mathfrak{s}$. But $\mathfrak{t}$ is fixed pointwise by $T$ and hence by each $\sigma_{i}$. Thus, $\mathfrak{t} \subseteq \mathfrak{g}=\mathfrak{s}^{\sigma}$, so $\mathfrak{t}$ is a Cartan subalgebra of $\mathfrak{g}$. Hence the simple Lie algebras $\mathfrak{g}$ and $\mathfrak{s}$ have the same rank, so we have (e). Finally, to prove "(e) $\Rightarrow(\mathrm{a})$ ", suppose that $\mathfrak{h}$ is a Cartan subalgebra of $\mathfrak{s}$. Then $\Delta$ is reduced and therefore, by (25), $\Delta=\Delta_{\mathfrak{g}}$. Next, if $\alpha \in \Delta^{\times}=\Delta_{\mathfrak{g}}^{\times}$, we have $0 \neq \mathfrak{g}_{\alpha} \subseteq \mathfrak{s}_{\alpha}$, so, $\mathfrak{g}_{\alpha}=\mathfrak{s}_{\alpha} \operatorname{since} \operatorname{dim}\left(\mathfrak{s}_{\alpha}\right)=1$. Thus, $\mathfrak{s} \subseteq \mathfrak{g}$, and hence $\mathfrak{s}=\mathfrak{g}$, which implies (a).

Remark 4.1.7. Suppose that $\mathfrak{s}$ is a finite dimensional simple Lie algebra, $\boldsymbol{\sigma}=$ $\left(\sigma_{1}, \ldots, \sigma_{n}\right) \in \operatorname{cfo}_{n}(\mathfrak{s})$ and $\mathbf{m} \in \mathbb{Z}_{+}^{n}$ with $\boldsymbol{\sigma}^{\mathbf{m}}=\mathbf{1}$. If the automorphisms $\sigma_{1}, \ldots, \sigma_{n}$ are contained in a common torus of $\operatorname{Aut}_{k}(\mathfrak{s})$, it is not difficult to show using techniques of Galois cohomology that the multiloop algebra $M_{\mathbf{m}}(\mathfrak{s}, \boldsymbol{\sigma})$ is isomorphic as a Lie algebra to the untwisted multiloop algebra $\mathfrak{s} \otimes k\left[z_{1}^{ \pm 1}, \ldots, z_{n}^{ \pm 1}\right]$. We have not used that general fact in the graded result, Corollary 4.1.6. 
4.2. Isotopy of multiloop Lie tori. Throughout this subsection suppose that $\mathfrak{s}$ is a finite dimensional simple Lie algebra over $k, \boldsymbol{\sigma}=\left(\sigma_{1}, \ldots, \sigma_{n}\right)$ is a sequence in $\operatorname{cfo}_{n}(\mathfrak{s})$ satisfying (A1)-(A3), and $\mathfrak{h}$ is a Cartan subalgebra of $\mathfrak{g}=\mathfrak{s}^{\sigma}$. Let $\mathbf{m}=\left(m_{1}, \ldots, m_{n}\right)$, where $m_{i}=\left|\sigma_{i}\right|, \Delta=\Delta(\mathfrak{s}, \mathfrak{h})$ and $Q=\operatorname{span}_{\mathbb{Z}}(\Delta)$, and let $\mathfrak{s}=\bigoplus_{\alpha \in Q} \mathfrak{s}_{\alpha}$ be the root space decomposition of $\mathfrak{s}$ relative to the adjoint action of $\mathfrak{h}$. Finally, let

$$
\mathcal{L}=\operatorname{LT}(\mathfrak{s}, \boldsymbol{\sigma}, \mathfrak{h})
$$

be the multiloop Lie $\mathbb{Z}^{n}$-torus determined by $\mathfrak{s}, \boldsymbol{\sigma}$ and $\mathfrak{h}$.

We will be considering isotopes of $\mathcal{L}$. (See subsection 2.2 for the terminology and notation used here.) To do this we use elements $s \in \operatorname{Hom}\left(Q, \mathbb{Z}^{n}\right)$, which we write as $s=\left(s_{1}, \ldots, s_{n}\right)$, where $s_{i} \in \operatorname{Hom}(Q, \mathbb{Z})$ for $1 \leq i \leq n$.

Proposition 4.2.1. Suppose that $s=\left(s_{1}, \ldots, s_{n}\right) \in \operatorname{Hom}\left(Q, \mathbb{Z}^{n}\right)$ is admissible for $\mathcal{L}$. For $1 \leq i \leq n$, define $\tau_{i}$ and $\tilde{\sigma}_{i}$ in $\operatorname{Aut}_{k}(\mathfrak{s})$ by

$$
\tau_{i}\left(x_{\alpha}\right)=\zeta_{m_{i}}^{-s_{i}(\alpha)} x_{\alpha}
$$

for $\alpha \in Q, x_{\alpha} \in \mathfrak{s}_{\alpha}$, and

$$
\tilde{\sigma}_{i}=\tau_{i} \sigma_{i}=\sigma_{i} \tau_{i} \in \operatorname{Aut}_{k}(\mathfrak{s})
$$

for $1 \leq i \leq n$. Then $\tilde{\boldsymbol{\sigma}}=\left(\tilde{\sigma}_{1}, \ldots, \tilde{\sigma}_{n}\right) \in \operatorname{cfo}_{n}(\mathfrak{s})$, $\tilde{\boldsymbol{\sigma}}$ satisfies (A1)-(A3), $\mathfrak{h}$ is a Cartan subalgebra of $\mathfrak{s}^{\tilde{\sigma}}$, $\left|\tilde{\sigma}_{i}\right|=m_{i}$ for $1 \leq i \leq n$, and $\mathcal{L}^{(s)}$ is $Q \times \mathbb{Z}^{n}$-graded isomorphic to $\operatorname{LT}(\mathfrak{s}, \tilde{\boldsymbol{\sigma}}, \mathfrak{h})$.

Proof. It is clear that the automorphisms $\sigma_{1}, \ldots, \sigma_{n}, \tau_{1}, \ldots, \tau_{n}$ commute. So our definition of $\tilde{\sigma}_{i}$ makes sense, the automorphisms $\tilde{\sigma}_{1}, \ldots, \tilde{\sigma}_{n}$ commute, $\tilde{\sigma}_{i}^{m_{i}}=1$ and $\mathfrak{h}$ is fixed pointwise by each $\tilde{\sigma}_{i}$. So, using Construction 3.2.1, we can form $\widetilde{\mathcal{L}}=\operatorname{LT}_{\mathbf{m}}(\mathfrak{s}, \mathfrak{h}, \tilde{\boldsymbol{\sigma}})$.

Now we have

$$
\mathcal{L}=\sum_{(\alpha, \lambda) \in Q \times \mathbb{Z}^{n}} \mathfrak{s}_{\alpha}^{\bar{\lambda}} \otimes z^{\lambda}
$$

where $\mathfrak{s}$ is $Q \times \bar{\Lambda}$-graded with the $Q$-grading determined by $\mathfrak{h}$ and the $\bar{\Lambda}$-grading determined by $\boldsymbol{\sigma}$. In contrast, we have

$$
\widetilde{\mathcal{L}}=\sum_{(\alpha, \lambda) \in Q \times \mathbb{Z}^{n}} \tilde{\mathfrak{s}}_{\alpha}^{\bar{\lambda}} \otimes z^{\lambda},
$$

where we use the notation $\tilde{\mathfrak{s}}$ for the $Q \times \bar{\Lambda}$-graded algebra $\mathfrak{s}$ with the $Q$-grading determined by $\mathfrak{h}$ and the $\bar{\Lambda}$-grading determined by $\tilde{\boldsymbol{\sigma}}$. One checks directly using the definitions that

$$
\tilde{\mathfrak{s}}_{\alpha}^{\bar{\lambda}}=\mathfrak{s}_{\alpha}^{\overline{\lambda+s(\alpha)}}
$$

Note that $\mathcal{L}, \mathcal{L}^{(s)}$ and $\widetilde{\mathcal{L}}$ are all subalgebras of $\mathfrak{s} \otimes k\left[z_{1}^{ \pm 1}, \ldots, z_{n}^{ \pm 1}\right]$. We define a $k$-algebra automorphism $\psi$ of $\mathfrak{s} \otimes k\left[z_{1}^{ \pm 1}, \ldots, z_{n}^{ \pm 1}\right]$ by

$$
\psi\left(x_{\alpha} \otimes a\right)=x_{\alpha} \otimes a z^{-s(\alpha)}
$$

for $x_{\alpha} \in \mathfrak{s}_{\alpha}, \alpha \in Q, a \in k\left[z_{1}^{ \pm 1}, \ldots, z_{n}^{ \pm 1}\right]$. Then, for $\alpha \in Q, \lambda \in \mathbb{Z}^{n}$, we have

$$
\psi\left(\left(\mathcal{L}^{(s)}\right)_{\alpha}^{\lambda}\right)=\psi\left(\mathcal{L}_{\alpha}^{\lambda+s(\alpha)}\right)=\psi\left(\mathfrak{s}_{\alpha}^{\overline{\lambda+s(\alpha)}} \otimes z^{\lambda+s(\alpha)}\right)=\mathfrak{s}_{\alpha}^{\overline{\lambda+s(\alpha)}} \otimes z^{\lambda}=\tilde{\mathfrak{s}}_{\alpha}^{\bar{\lambda}} \otimes z^{\lambda}=\widetilde{\mathcal{L}}_{\alpha}^{\lambda} .
$$

So $\psi$ restricts to a $Q \times \Lambda$-graded isomorphism of $\mathcal{L}^{(s)}$ onto $\widetilde{\mathcal{L}}$.

Next, by Proposition 3.2.5 $\mathcal{L}$ is a Lie torus. Hence, since $s$ is admissible for $\mathcal{L}$, $\mathcal{L}^{(s)}$ is a Lie torus and therefore so is $\widetilde{\mathcal{L}}$. Thus, by Proposition 3.2.5, $\tilde{\boldsymbol{\sigma}}$ satisfies (A1)-(A3), $\mathfrak{h}$ is a Cartan subalgebra of $\mathfrak{s}^{\tilde{\sigma}}$, and $\left|\tilde{\sigma}_{i}\right|=m_{i}$ for $1 \leq i \leq n$. 
Definition 4.2.2. As in [KW, §1.10], we define $\operatorname{Ad}: \operatorname{Hom}\left(Q, k^{\times}\right) \rightarrow \operatorname{Aut}_{k}(\mathfrak{s})$ by

$$
\operatorname{Ad}(\rho)\left(x_{\alpha}\right)=\rho(\alpha) x_{\alpha}
$$

for $x_{\alpha} \in \mathfrak{s}_{\alpha}, \alpha \in Q$. Then $\operatorname{Ad}$ is a group homomorphism, so $\operatorname{Ad}\left(\operatorname{Hom}\left(Q, k^{\times}\right)\right)$is a subgroup of $\operatorname{Aut}_{k}(\mathfrak{s})$.

Remark 4.2.3. $\operatorname{Aut}_{k}(\mathfrak{s})$ is an algebraic group with Lie algebra $\operatorname{ad}_{\mathfrak{s}}(\mathfrak{s})$, and Ad is a homomorphism of algebraic groups, so $H=\operatorname{Ad}\left(\operatorname{Hom}\left(Q, k^{\times}\right)\right)$is a closed connected subgroup of $\operatorname{Aut}_{k}(\mathfrak{s})$. In fact, it is not difficult to show that $H$ is the torus in $\operatorname{Aut}_{k}(\mathfrak{s})$ whose Lie algebra is $\operatorname{ad}_{\mathfrak{s}}(\mathfrak{h})$. We omit the proof of this fact, since we won't use it.

Lemma 4.2.4. Let $H=\operatorname{Ad}\left(\operatorname{Hom}\left(Q, k^{\times}\right)\right)$and let $\Pi$ be a base for $\Delta$. Suppose $\boldsymbol{\tau}=\left(\tau_{1}, \ldots, \tau_{n}\right) \in H^{n}$ with $\boldsymbol{\tau}^{\mathbf{m}}=\mathbf{1}$, and let $\tilde{\boldsymbol{\sigma}}=\left(\tilde{\sigma}_{1}, \ldots, \tilde{\sigma}_{n}\right) \in \operatorname{cfo}_{n}(\mathfrak{s})$, where $\tilde{\sigma}_{i}=\tau_{i} \sigma_{i}$ for $1 \leq i \leq n$. Then the following are equivalent:

(a) $\tilde{\boldsymbol{\sigma}}$ satisfies (A1)-(A3);

(b) $\tilde{\boldsymbol{\sigma}}^{P}$ satisfies (A1)-(A3) for some $P \in \mathrm{GL}_{n}(\mathbb{Z})$;

(c) $\mathfrak{s}^{\tilde{\sigma}} \cap \mathfrak{s}_{\alpha} \neq 0$ for $\alpha \in \Delta$;

(d) $\mathfrak{s}^{\tilde{\boldsymbol{\sigma}}} \cap \mathfrak{s}_{\alpha} \neq 0$ for $\alpha \in \Pi$.

Further, in that case, $\mathfrak{h}$ is a Cartan subalgebra of $\mathfrak{s}^{\tilde{\boldsymbol{\sigma}}}$ and $\mathcal{L}$ is isotopic to $\operatorname{LT}(\mathfrak{s}, \tilde{\boldsymbol{\sigma}}, \mathfrak{h})$.

Proof. "(a) $\Rightarrow$ (b)" is trivial.

"(b) $\Rightarrow$ (c)" Let $\boldsymbol{\sigma}^{\prime}=\left(\sigma_{1}^{\prime}, \ldots, \sigma_{n}^{\prime}\right)=\tilde{\boldsymbol{\sigma}}^{P}$, so by assumption $\boldsymbol{\sigma}^{\prime}$ satisfies (A1)(A3). Now, by (20), we have $\mathfrak{s}^{\sigma^{\prime}}=\mathfrak{s}^{\tilde{\sigma}}$, and we let

$$
\mathfrak{g}=\mathfrak{s}^{\sigma} \quad \text { and } \quad \mathfrak{g}^{\prime}=\mathfrak{s}^{\sigma^{\prime}}=\mathfrak{s}^{\tilde{\sigma}} .
$$

Since $\boldsymbol{\sigma}$ and $\boldsymbol{\sigma}^{\prime}$ satisfy (A1), $\mathfrak{g}$ and $\mathfrak{g}^{\prime}$ are simple.

Next $\mathfrak{h}$ is a Cartan subalgebra of $\mathfrak{g}$ and $\mathfrak{h} \subseteq \mathfrak{s}^{\tilde{\sigma}}=\mathfrak{g}^{\prime}$. So $\mathfrak{h}$ is contained in a Cartan subalgebra $\mathfrak{h}^{\prime}$ of $\mathfrak{g}^{\prime}$. Let $\pi: \mathfrak{h}^{\prime *} \rightarrow \mathfrak{h}^{*}$ be the restriction map. Each root space $\mathfrak{s}_{\alpha}$ for the adjoint action of $\mathfrak{h}$ is stabilized by the adjoint action of $\mathfrak{h}^{\prime}$. Hence, we have

$$
\mathfrak{s}_{\alpha}=\sum_{\alpha^{\prime} \in \mathfrak{h}^{\prime *}, \pi\left(\alpha^{\prime}\right)=\alpha} \mathfrak{s}_{\alpha^{\prime}}
$$

for $\alpha \in \mathfrak{h}^{*}$, where $\mathfrak{s}_{\alpha^{\prime}}$ is the $\alpha^{\prime}$-root space of $\mathfrak{s}$ relative to the adjoint action of $\mathfrak{h}^{\prime}$. Consequently, since $\Delta=\Delta(\mathfrak{s}, \mathfrak{h})$, we have $\pi\left(\Delta\left(\mathfrak{s}, \mathfrak{h}^{\prime}\right)\right)=\Delta$, so

$$
\Delta_{\text {ind }}^{\times} \subseteq \pi\left(\Delta\left(\mathfrak{s}, \mathfrak{h}^{\prime}\right)_{\text {ind }}^{\times}\right) .
$$

Now let $\alpha \in \Delta_{\text {ind }}^{\times}$. Then, by (32), $\alpha \in \pi\left(\alpha^{\prime}\right)$, where $\alpha^{\prime} \in \Delta\left(\mathfrak{s}, \mathfrak{h}^{\prime}\right)_{\text {ind }}^{\times}$. Now, since $\boldsymbol{\sigma}^{\prime}$ satisfies (A1)-(A3), $\operatorname{LT}\left(\mathfrak{s}, \boldsymbol{\sigma}^{\prime}, \mathfrak{h}^{\prime}\right)$ is a Lie torus, so it satisfies (LT2)(i). Thus, $\operatorname{LT}\left(\mathfrak{s}, \boldsymbol{\sigma}^{\prime}, \mathfrak{h}^{\prime}\right)_{\alpha^{\prime}}^{0} \neq 0$, and therefore $\mathfrak{s}^{\boldsymbol{\sigma}^{\prime}} \cap \mathfrak{s}_{\alpha^{\prime}} \neq 0$ by (23). Since $\mathfrak{s}_{\alpha^{\prime}} \subseteq \mathfrak{s}_{\alpha}$, we have $\mathfrak{s}^{\boldsymbol{\sigma}^{\prime}} \cap \mathfrak{s}_{\alpha} \neq 0$, so $\mathfrak{s}^{\tilde{\sigma}} \cap \mathfrak{s}_{\alpha} \neq 0$.

"(c) $\Rightarrow$ (d)" is trivial.

"(d) $\Rightarrow$ (a)" If $1 \leq i \leq n$, then $\tau_{i}^{m_{i}}=1$ and $\tau_{i}=\operatorname{Ad}\left(\rho_{i}\right)$ for some $\rho_{i} \in$ $\operatorname{Hom}\left(Q, k^{\times}\right)$. Hence, $\rho_{i}(\alpha)^{m_{i}}=1$ for $\alpha \in \Delta$ and $1 \leq i \leq n$. Thus, we may choose $s_{i} \in \operatorname{Hom}(Q, \mathbb{Z})$ such that $\rho_{i}(\alpha)=\zeta_{m_{i}}^{-s_{i}(\alpha)}$ for $\alpha \in Q$. (Choose $s_{i}$ so that this holds for $\alpha$ in $\Pi$, in which case it holds for all $\alpha \in Q$.) So

$$
\tau_{i}(x)=\zeta_{m_{i}}^{-s_{i}(\alpha)} x
$$

for $x \in \mathfrak{s}_{\alpha}, \alpha \in Q$. Let $s=\left(s_{1}, \ldots, s_{n}\right) \in \operatorname{Hom}\left(Q, \mathbb{Z}^{n}\right)$. 
To show that $s$ is admissible for $\mathcal{L}$, let $\alpha \in \Pi$. By assumption, we may choose $0 \neq x \in \mathfrak{s}^{\tilde{\boldsymbol{\sigma}}} \cap \mathfrak{s}_{\alpha}$. Thus, $\sigma_{i} \tau_{i}(x)=x$, so $\sigma_{i}(x)=\zeta_{m_{i}}^{s_{i}(\alpha)} x$. for all $i$. Hence $x \in \mathfrak{s}_{\alpha}^{s(\alpha)}$, so $\mathcal{L}_{\alpha}^{s(\alpha)} \neq 0$ by (23). Hence, $s(\alpha) \in \Lambda_{\alpha}$, and $s$ is admissible for $\mathcal{L}$.

So, by Proposition 4.2.1. (a) holds, $\mathfrak{h}$ is a Cartan subalgebra for $\mathfrak{s}^{\tilde{\boldsymbol{\sigma}}}$, and $\mathcal{L}$ is isotopic to $\operatorname{LT}(\mathfrak{s}, \tilde{\sigma}, \mathfrak{h})$.

Theorem 4.2.5. Let $\mathcal{L}=\operatorname{LT}(\mathfrak{s}, \boldsymbol{\sigma}, \mathfrak{h})$ and $\mathcal{L}^{\prime}=\operatorname{LT}\left(\mathfrak{s}^{\prime}, \boldsymbol{\sigma}^{\prime}, \mathfrak{h}^{\prime}\right)$ be multiloop Lie $\mathbb{Z}^{n}$-tori, let $\Delta=\Delta(\mathfrak{s}, \mathfrak{h}), Q=Q(\Delta), m_{i}=\left|\sigma_{i}\right|$, and let $H=\operatorname{Ad}\left(\operatorname{Hom}\left(Q, k^{\times}\right)\right)$. Then, $\mathcal{L}$ is isotopic to $\mathcal{L}^{\prime}$ if and only if there exist $\tau_{1}, \ldots, \tau_{n} \in H$ with $\tau_{j}^{m_{j}}=1$, $P=\left(p_{i j}\right) \in \mathrm{GL}_{n}(\mathbb{Z})$ and an algebra isomorphism $\varphi: \mathfrak{s} \rightarrow \mathfrak{s}^{\prime}$ such that

$$
\boldsymbol{\sigma}^{\prime}=\varphi \tilde{\boldsymbol{\sigma}}^{P} \varphi^{-1}
$$

where $\tilde{\sigma}=\left(\tau_{1} \sigma_{1}, \ldots, \tau_{n} \sigma_{n}\right)$.

Proof. " $\Rightarrow$ " Suppose that $\mathcal{L}$ is isotopic to $\mathcal{L}^{\prime}$. Then, $\mathcal{L}^{(s)}$ is bi-isomorphic to $\mathcal{L}^{\prime}$ for some $s=\left(s_{1}, \ldots, s_{n}\right) \in \operatorname{Hom}\left(Q, \mathbb{Z}^{n}\right)$ that is admissible for $\mathcal{L}$. Let $\tau_{i}$ and $\tilde{\boldsymbol{\sigma}}$ be defined as in Proposition 4.2.1, Then $\tau_{i}=\operatorname{Ad}\left(\rho_{i}\right)$, where $\rho_{i} \in \operatorname{Hom}\left(Q, k^{\times}\right)$is defined by $\rho_{i}(\alpha)=\zeta_{m_{i}}^{-s_{i}(\alpha)}$. So $\tau_{i} \in H$ and $\tau_{i}^{m_{i}}=1$ for all $i$. But, by Proposition 4.2.1, $\mathcal{L}^{(s)}$ is $Q \times \Lambda$-graded isomorphic to $\widetilde{\mathcal{L}}=\operatorname{LT}(\mathfrak{s}, \tilde{\boldsymbol{\sigma}}, \mathfrak{h})$. Hence, $\widetilde{\mathcal{L}}$ is bi-isomorphic to $\mathcal{L}^{\prime}$, and our conclusion follows from Theorem 4.1.1.

" $\Leftarrow "$ Since $\boldsymbol{\sigma}^{\prime}$ satisfies (A1)-(A3), $\tilde{\boldsymbol{\sigma}}^{P}=\varphi^{-1} \boldsymbol{\sigma}^{\prime} \varphi$ satisfies (A1)-(A3). So, by Lemma 4.2.4, $\tilde{\boldsymbol{\sigma}}$ satisfies (A1)-(A3), $\mathfrak{h}$ is a Cartan subalgebra of $\mathfrak{s}^{\tilde{\boldsymbol{\sigma}}}$, and $\mathcal{L}$ is isotopic to $\operatorname{LT}(\mathfrak{s}, \tilde{\boldsymbol{\sigma}}, \mathfrak{h})$. But, by Theorem 4.1.1. $\operatorname{LT}(\mathfrak{s}, \tilde{\boldsymbol{\sigma}}, \mathfrak{h})$ is bi-isomorphic to $\mathcal{L}^{\prime}$. So $\mathcal{L}$ is isotopic to $\mathcal{L}^{\prime}$.

Corollary 4.2.6. If a multiloop Lie $\mathbb{Z}^{n}$-torus $\operatorname{LT}(\mathfrak{s}, \boldsymbol{\sigma}, \mathfrak{h})$ is isotopic to an untwisted multiloop Lie $\mathbb{Z}^{n}$-torus, then $\boldsymbol{\sigma}=\mathbf{1}$. Two untwisted multiloop Lie $\mathbb{Z}^{n}$-tori $\operatorname{LT}(\mathfrak{s}, \mathbf{1}, \mathfrak{h})$ and $\operatorname{LT}\left(\mathfrak{s}^{\prime}, \mathbf{1}, \mathfrak{h}^{\prime}\right)$ are isotopic if and only if $\mathfrak{s}$ and $\mathfrak{s}^{\prime}$ are isomorphic.

Proof. The second statement follows immediately from Theorem 4.2.5. For the first statement, suppose that $\operatorname{LT}(\mathfrak{s}, \boldsymbol{\sigma}, \mathfrak{h})$ is isotopic to $\operatorname{LT}\left(\mathfrak{s}^{\prime}, \mathbf{1}, \mathfrak{h}^{\prime}\right)$. Choose $\tau_{1}, \ldots, \tau_{n}, P$, $\tilde{\boldsymbol{\sigma}}$ and $\varphi$ as in Theorem 4.2.5. Then, $\tilde{\boldsymbol{\sigma}}^{P}=\varphi^{-1} \mathbf{1} \varphi=\mathbf{1}$, so $\tilde{\boldsymbol{\sigma}}=1$. Thus, $\sigma_{1}, \ldots, \sigma_{n}$ are contained in the torus $H$, so, by Corollary 4.1.6, $\boldsymbol{\sigma}=\mathbf{1}$.

Corollary 4.2.7. Two (multi)loop Lie $\mathbb{Z}$-tori $\operatorname{LT}(\mathfrak{s}, \sigma, \mathfrak{h})$ and $\operatorname{LT}\left(\mathfrak{s}^{\prime}, \sigma^{\prime}, \mathfrak{h}^{\prime}\right)$ are isotopic if and only if they are bi-isomorphic.

Proof. Fix a Cartan subalgebra $\mathfrak{t}$ of $\mathfrak{s}$ and an épinglage $\left(\left\{\alpha_{i}\right\},\left\{e_{i}\right\}\right)$ of $(\mathfrak{s}, \mathfrak{t})$. Let $D$ denote the Dynkin diagram of $\mathfrak{s}$ with respect to $\mathfrak{t}$ and $\left\{\alpha_{i}\right\}$. Then $\operatorname{Aut}(D)$ can be identified with the group of diagram automorphisms of $\mathfrak{s}$ with respect to $\mathfrak{t}$ and $\left(\left\{\alpha_{i}\right\},\left\{e_{i}\right\}\right)$, and we have $\operatorname{Aut}(\mathfrak{s})=\operatorname{Aut}(\mathfrak{s})^{0} \rtimes \operatorname{Aut}(D)$ [B2, Chap. VIII, $\left.\S 5\right]$.

Now one direction in the corollary is trivial, so we assume that $\operatorname{LT}(\mathfrak{s}, \sigma, \mathfrak{h})$ and $\operatorname{LT}\left(\mathfrak{s}^{\prime}, \sigma^{\prime}, \mathfrak{h}^{\prime}\right)$ are isotopic. By Proposition 3.4.1 and Theorem 4.1.1. we can assume $\mathfrak{s}=\mathfrak{s}^{\prime}$ and $\sigma, \sigma^{\prime} \in \operatorname{Aut}(D)$. Further, by Theorem 4.2.5, we have $\sigma^{\prime}=\varphi \tau \sigma^{ \pm 1} \varphi^{-1}$, where $\varphi \in \operatorname{Aut}_{k}(\mathfrak{s})$ and $\tau \in \operatorname{Aut}(\mathfrak{s})^{0}$. Projecting this equation onto $\operatorname{Aut}(D)$ shows that $\sigma^{\prime}$ is conjugate to $\sigma^{ \pm 1}$ in $\operatorname{Aut}(D)$ and hence also in $\operatorname{Aut}_{k}(\mathfrak{s})$. Thus, $\operatorname{LT}(\mathfrak{s}, \sigma, \mathfrak{h})$ and $\operatorname{LT}\left(\mathfrak{s}^{\prime}, \sigma^{\prime}, \mathfrak{h}^{\prime}\right)$ are bi-isomorphic by Theorem 4.1.1.

4.3. An example. We expect that Corollary 4.2.7 is also true for multiloop Lie $\mathbb{Z}^{2}$-tori. However, it is not true for multiloop Lie $\mathbb{Z}^{3}$-tori, as the next example shows. 
Example 4.3.1. Let $f: k^{7} \times k^{7} \rightarrow k$ be the symmetric bilinear form with matrix $\operatorname{diag}\left(J_{3}, \mathrm{I}_{4}\right)$ relative to the standard basis, where $J_{3}=\left[\begin{array}{lll}0 & 0 & 1 \\ 0 & 1 & 0 \\ 1 & 0 & 0\end{array}\right]$ and $\mathrm{I}_{4}$ is the $4 \times 4$ identity matrix. Let $\mathfrak{s}=\mathfrak{o}(f)$ be the orthogonal Lie algebra of endomorphisms of $k^{7}$ that are skew relative to $f$, in which case $\mathfrak{s}$ is simple of type $\mathrm{B}_{3}$. Writing endomorphisms of $k^{7}$ as matrices relative to the standard basis, we see that $\mathfrak{s}$ is the Lie algebra of all matrices of the form

$$
\left[\begin{array}{cc}
A & -J_{3} C^{t} \\
C & B
\end{array}\right]
$$

where $A \in \operatorname{Mat}_{3}(k), B \in \operatorname{Mat}_{4}(k), C \in \operatorname{Mat}_{4 \times 3}(k), A^{t}=-J_{3} A J_{3}$ and $B^{t}=-B$. Also, if $\mathrm{SO}(f)$ denotes the special orthogonal group of $f$, the map $c \mapsto c_{g}$ is an isomorphism of $\mathrm{SO}(f)$ onto $\operatorname{Aut}_{k}(\mathfrak{s})$, where $c_{g}(x)=g x g^{-1}$ for $x \in \mathfrak{s}$ [J] Theorem IX.6].

Next let $\boldsymbol{\sigma}=\left(\sigma_{1}, \sigma_{2}, \sigma_{3}\right)=\left(c_{d_{1}}, c_{d_{2}}, c_{d_{3}}\right) \in \operatorname{cfo}_{3}(\mathfrak{s})$, where

$$
d_{1}=\operatorname{diag}\left(\mathrm{I}_{3},-1,1,1,-1\right), d_{2}=\operatorname{diag}\left(\mathrm{I}_{3}, 1,-1,1,-1\right), d_{3}=\operatorname{diag}\left(\mathrm{I}_{3}, 1,1,-1,-1\right)
$$

in $\mathrm{SO}(f)$. Then,

$$
\mathfrak{g}=\mathfrak{s}^{\sigma}=\left\{\left[\begin{array}{cc}
A & 0 \\
0 & 0
\end{array}\right] \mid A \in \operatorname{Mat}_{3}(k), A^{t}=-J_{3} A J_{3}\right\}
$$

is simple of type $\mathrm{A}_{1}$ with Cartan subalgebra $\mathfrak{h}=k\left(e_{11}-e_{33}\right)$, and we have $\Delta=$ $\Delta(\mathfrak{s}, \mathfrak{h})=\left\{\varepsilon_{1}, 0,-\varepsilon_{1}\right\}$, where $\varepsilon_{1}\left(e_{11}-e_{33}\right)=1$. So $Q=Q(\Delta)=\mathbb{Z} \varepsilon_{1}$.

Let $\mathbf{m}=(2,2,2)$ and, as in Construction 3.2.1, let $\bar{\Lambda}=(\mathbb{Z} /(2))^{3}$. Then, one checks that for $\overline{0} \neq \bar{\lambda} \in \bar{\Lambda}$, the $\mathfrak{g}$ module $\mathfrak{s}^{\bar{\lambda}}$ is isomorphic to the adjoint module in 4 cases, whereas $\mathfrak{s}^{\bar{\lambda}}$ is 2 -dimensional with trivial action in the other 3 cases. So $\boldsymbol{\sigma}$ satisfies (A1) and (A2), and, since $|\langle\boldsymbol{\sigma}\rangle|=8, \boldsymbol{\sigma}$ satisfies (A3). Thus, by Proposition 3.2.5. $\mathcal{L}=\operatorname{LT}(\mathfrak{s}, \boldsymbol{\sigma}, \mathfrak{h})$ is a multiloop Lie $\mathbb{Z}^{3}$-torus of type $\Delta$.

Now let $H=\operatorname{Ad}\left(\operatorname{Hom}\left(Q, k^{\times}\right)\right)$, in which case $H=\left\{c_{\operatorname{diag}\left(a, 1, a^{-1}, \mathrm{I}_{4}\right)} \mid a \in k^{\times}\right\}$. Put

$$
d=\operatorname{diag}\left(-1,1,-1, \mathrm{I}_{4}\right) \in \mathrm{SO}(f), \quad \tau_{1}=\tau_{2}=\tau_{3}=c_{d},
$$

$\boldsymbol{\tau}=\left(\tau_{1}, \tau_{2}, \tau_{3}\right)$ and $\tilde{\boldsymbol{\sigma}}=\left(\tilde{\sigma}_{1}, \tilde{\sigma}_{2}, \tilde{\sigma}_{3}\right)$ with $\tilde{\sigma}_{i}=\tau_{i} \sigma_{i}$ for $i=1,2,3$. Then, one checks that $e_{71}-e_{37} \in \mathfrak{s}^{\tilde{\boldsymbol{\sigma}}} \cap \mathfrak{s}_{\varepsilon_{1}}$. So by Lemma 4.2.4, $\tilde{\boldsymbol{\sigma}}$ satisfies (A1)-(A3), $\mathfrak{h}$ is a Cartan subalgebra of $\mathfrak{s}^{\tilde{\sigma}}$, and $\mathcal{L}$ is isotopic to $\tilde{\mathcal{L}}=\operatorname{LT}(\mathfrak{s}, \tilde{\boldsymbol{\sigma}}, \mathfrak{h})$.

Note finally that $\langle\boldsymbol{\sigma}\rangle=c_{D}$ and $\langle\tilde{\boldsymbol{\sigma}}\rangle=c_{\tilde{D}}$, where $D=\left\langle d_{1}, d_{2}, d_{3}\right\rangle$ and $\tilde{D}=$ $\left\langle d d_{1}, d d_{2}, d d_{3}\right\rangle$. Then, $\tilde{D}$ contains an element $\left(d d_{1}\right)\left(d d_{2}\right)\left(d d_{3}\right)$ with (-1)-eigenspace of dimension 6 , whereas $D$ clearly contains no such element. Thus, $D$ and $\tilde{D}$ are not conjugate in $\mathrm{SO}(f)$, so $\langle\boldsymbol{\sigma}\rangle$ and $\langle\tilde{\boldsymbol{\sigma}}\rangle$ are not conjugate in $\operatorname{Aut}_{k}(\mathfrak{s})$. Hence, by Corollary 4.1.4 $\mathcal{L}$ and $\tilde{\mathcal{L}}$ are not bi-isomorphic.

Remark 4.3.2. Suppose that we have the assumptions and notation of Example 4.3.1 with just one change: we let $\tau_{1}=\tau_{2}=c_{d}$ and $\tau_{3}=\mathrm{id}$. In this case $\mathfrak{s}^{\tilde{\boldsymbol{\sigma}}} \cap \mathfrak{s}_{\varepsilon_{1}}=0$, so, by Lemma 4.2.4, $\tilde{\boldsymbol{\sigma}}$ does not satisfy (A1)-(A3). In fact, $\mathfrak{s}^{\tilde{\boldsymbol{\sigma}}}=k\left(e_{11}-e_{33}\right)$, which is not simple.

Remark 4.3.3. Example 4.3.1 is presented in a very different way in AF, Example 8.9], where $\mathcal{L}$ and $\mathcal{L}^{\prime}$ are constructed using the Tits-Kantor-Koecher Lie algebra construction from spin-factor Jordan tori $\mathcal{J}$ and $\mathcal{J}^{\prime}$ that are isotopic but not isograded-isomorphic. (See also Remark 2.2.5 above.) 


\section{An APPROACH TO ClASSIFICATION}

In this section we describe an approach to the classification of fgc Lie tori first up to bi-isomorphism and then up to isotopy.

5.1. A proposition about finite abelian groups. In this subsection, we prove a proposition about finite abelian groups. (This will be applied in subsection 5.3 to the group generated by a sequence of commuting finite order automorphisms.) We will prove this proposition using the following lemma about integral matrices.

Lemma 5.1.1. Suppose that $m_{1}, \ldots, m_{n}$ are positive integers and $m_{i+1}$ divides $m_{i}$ for $1 \leq i<n$. Let $\mathcal{M}$ be the right ideal generated by $\operatorname{diag}\left(m_{1}, \ldots, m_{n}\right)$ in $\operatorname{Mat}_{n}(\mathbb{Z})$, the ring of $n \times n$ matrices over $\mathbb{Z}$. If $A, B \in \operatorname{Mat}_{n}(\mathbb{Z})$ with

$$
A B \equiv \mathrm{I}_{n} \quad(\bmod \mathcal{M})
$$

then there is $P \in \mathrm{GL}_{n}(\mathbb{Z})$ with $A P \equiv \operatorname{diag}(1, \ldots, 1, p)(\bmod \mathcal{M})$, where

$$
0 \leq p \leq\left\lfloor\frac{m_{n}}{2}\right\rfloor \quad \text { and } \operatorname{gcd}\left(p, m_{n}\right)=1
$$

(so $p=0$ if $m_{n}=1$ ). Moreover, $p$ is uniquely determined by the equivalence class of $A(\bmod \mathcal{M})$.

Proof. We first note that $\left(a_{i j}\right) \equiv\left(a_{i j}^{\prime}\right)(\bmod \mathcal{M})$ if and only if $a_{i j} \equiv a_{i j}^{\prime}\left(\bmod m_{i}\right)$ for all $i, j$. Since $m_{n}$ divides $m_{i}$, we see that $\operatorname{det}(A)\left(\bmod m_{n}\right)$ is an invariant of the equivalence class of $A(\bmod \mathcal{M})$. Now if $A P \equiv \operatorname{diag}(1, \ldots, 1, p)(\bmod \mathcal{M})$ and $P \in \mathrm{GL}_{n}(\mathbb{Z})$, then $p \equiv \operatorname{det}(A P) \equiv \pm \operatorname{det}(A)\left(\bmod m_{n}\right)$ and $0 \leq p \leq\left\lfloor\frac{m_{n}}{2}\right\rfloor$ uniquely determine $p$.

To show the first statement, we shall repeatedly adjust $A$ by replacing $A$ by some $A^{\prime} \equiv A P(\bmod \mathcal{M})$ and $B$ by $P^{-1} B$ for some $P \in \mathrm{GL}_{n}(\mathbb{Z})$ until $A$ has the form $\operatorname{diag}(1, \ldots, 1, p)$, where $p$ satisfies (33). We suppose $A=\left(a_{i j}\right)$ is of the form $\left[\begin{array}{cc}\mathrm{I}_{k-1} & 0 \\ * & *\end{array}\right]$ and use induction on $k$. We can replace $a_{k k}$ by $a_{k k}+m_{k}$ to assume $a_{k k} \neq 0$. Let $d$ be the positive gcd of $a_{k k}, \ldots, a_{k n}$. Since elementary column operations are achieved by right multiplying by an element of $\mathrm{GL}_{n}(\mathbb{Z})$, we can adjust columns $k$ to $n$ to assume $a_{k k}=d$ and $a_{k j}=0$ for $k<j \leq n$. Moreover, if $k<n$, we can then take $a_{k, n}=m_{k}$, get a new gcd, and again adjust columns $k$ to $n$ to assume $d \mid m_{k}$. Let $B=\left(b_{i j}\right)$. The first $k$ entries in the $k$ th column of $A B \equiv \mathrm{I}_{n}(\bmod \mathcal{M})$ give $b_{i k} \equiv 0\left(\bmod m_{i}\right)$ for $1 \leq i<k$ and

$$
a_{k 1} b_{1 k}+\ldots+a_{k, k-1} b_{k-1, k}+d b_{k k} \equiv 1 \quad\left(\bmod m_{k}\right) .
$$

Since $m_{k}$ divides $m_{i}$ for $i<k$, we have $b_{i k} \equiv 0\left(\bmod m_{k}\right)$. Thus, $d b_{k k} \equiv 1$ $\left(\bmod m_{k}\right)$; i.e., $\operatorname{gcd}\left(d, m_{k}\right)=1$. If $k<n$, then $d \mid m_{k}$, so $d=1$. In general, since $d b_{k k} \equiv 1\left(\bmod m_{k}\right)$, we can add multiples of the $k$ th column to the previous columns to get $a_{k j} \equiv 0\left(\bmod m_{k}\right)$ for $1 \leq j<k$; i.e., we can adjust $A$ to assume that $a_{k k}=d$ and $a_{k j}=0$ for $j \neq k$. If $k<n$, then $d=1$ and we have completed the induction step. If $k=n$, we can adjust $A$ by multiplying the $n$th column by -1 , if necessary, to get $d \equiv p\left(\bmod m_{n}\right)$ with $0 \leq p \leq\left\lfloor\frac{m_{n}}{2}\right\rfloor$.

Remark 5.1.2. Suppose that $G$ is a finite abelian group. Since $G$ is finite and abelian, we have $\operatorname{cfo}_{n}(G)=G^{n}$, so we have the right action $(\boldsymbol{\sigma}, P) \mapsto \boldsymbol{\sigma}^{P}$ of $\mathrm{GL}_{n}(\mathbb{Z})$ on $G^{n}$ (see Notation 3.1.1(i)). Next let

$$
\operatorname{mg}(G):=\text { minimum number of generators of } G \text {. }
$$


It is well known that $\operatorname{mg}(G)$ is also equal to the length of the sequence of invariant factors of $G$. Finally, let

$$
\mathrm{gs}_{n}(G):=\left\{\left(\sigma_{1}, \ldots, \sigma_{n}\right) \in G^{n} \mid G=\left\langle\sigma_{1}, \ldots, \sigma_{n}\right\rangle\right\} .
$$

If $\operatorname{mg}(G) \leq n$, then $\operatorname{gs}_{n}(G) \neq \emptyset$ and, by (20), the right action of $\mathrm{GL}_{n}(\mathbb{Z})$ on $G^{n}$ restricts to a right action of $\mathrm{GL}_{n}(\mathbb{Z})$ on $\mathrm{gs}_{n}(G)$.

We now determine representatives of the orbits of the action of $\mathrm{GL}_{n}(\mathbb{Z})$ on $\mathrm{gs}_{n}(G)$.

Proposition 5.1.3. Suppose that $G$ is a finite abelian group and $r:=\operatorname{mg}(G) \leq n$. Choose $\boldsymbol{\tau}=\left(\tau_{1}, \ldots, \tau_{n}\right) \in G^{n}$ such that $G=\left\langle\tau_{1}\right\rangle \times \cdots \times\left\langle\tau_{n}\right\rangle$ (as an internal direct product) and $\left|\tau_{i+1}\right|$ divides $\left|\tau_{i}\right|$ for $1 \leq i<n$. (So the decreasing sequence of invariant factors of $G$ is $\left|\tau_{1}\right|, \ldots,\left|\tau_{r}\right|$, and we have $\left|\tau_{i}\right|=1$ for $r<i \leq n$.) Then

$$
\left(\tau_{1}, \ldots, \tau_{n-1}, \tau_{n}^{p}\right)
$$

where

$$
0 \leq p \leq\left\lfloor\frac{\left|\tau_{n}\right|}{2}\right\rfloor \quad \text { and } \operatorname{gcd}\left(p,\left|\tau_{n}\right|\right)=1,
$$

is a nonredundant list of representatives of the orbits of $\mathrm{GL}_{n}(\mathbb{Z})$ acting on $\mathrm{gs}_{n}(G)$. (If $r<n$, then $\left|\tau_{n}\right|=1$ and the only choice for $p$ is $p=0$.) Consequently, every orbit contains an element $\left(\sigma_{1}, \ldots, \sigma_{n}\right)$ with the property that $|G|=\left|\sigma_{1}\right| \cdots\left|\sigma_{n}\right|$. Also, if $r<n$ or if the smallest invariant factor of $G$ is less than or equal to 4, then $\mathrm{GL}_{n}(\mathbb{Z})$ acts transitively on $\operatorname{gs}_{n}(S)$.

Proof. It suffices to prove the first statement. Every $\sigma \in G^{n}$ can be written as $\boldsymbol{\sigma}=\boldsymbol{\tau}^{A}$ for some $A \in \operatorname{Mat}_{n}(\mathbb{Z})$. Also, $\boldsymbol{\tau}^{A}=\boldsymbol{\tau}^{A^{\prime}}$ if and only if $A \equiv A^{\prime}(\bmod \mathcal{M})$, where $\mathcal{M}$ is the right ideal in $\operatorname{Mat}_{n}(\mathbb{Z})$ generated by $\operatorname{diag}\left(\left|\tau_{1}\right|, \ldots,\left|\tau_{n}\right|\right)$.

Now suppose that $\boldsymbol{\sigma} \in \mathrm{gs}_{n}(G)$ and write $\boldsymbol{\sigma}=\boldsymbol{\tau}^{A}$, where $A \in \operatorname{Mat}_{n}(\mathbb{Z})$. Then $\boldsymbol{\tau}=\boldsymbol{\sigma}^{B}$ for some $B \in \operatorname{Mat}_{n}(\mathbb{Z})$. So $\boldsymbol{\tau}=\boldsymbol{\tau}^{A B}$ and hence $A B \equiv \mathrm{I}_{n}(\bmod \mathcal{M})$. Then by Lemma 5.1.1, there exists $P \in \mathrm{GL}_{n}(\mathbb{Z})$ such that $A P \equiv \operatorname{diag}(1, \ldots, 1, p)$ $(\bmod \mathcal{M})$, where $p$ satisfies (34). So $\boldsymbol{\sigma}^{P}=\boldsymbol{\tau}^{A P}=\left(\tau_{1}, \ldots, \tau_{n}^{p}\right)$.

Finally, suppose that $\left(\tau_{1}, \ldots, \tau_{n}^{p_{1}}\right)^{P}=\left(\tau_{1}, \ldots, \tau_{n}^{p_{2}}\right)$ for some $P \in \mathrm{GL}_{n}(\mathbb{Z})$, where $p_{1}, p_{2}$ satisfy (34). Then, $\operatorname{diag}\left(1, \ldots, 1, p_{1}\right) P \equiv \operatorname{diag}\left(1, \ldots, 1, p_{2}\right)(\bmod \mathcal{M})$, so $p_{1}=$ $p_{2}$ by Lemma 5.1.1.

5.2. Absolute type. We next recall some facts about absolute type from $\mathrm{ABP}$ (see also [N2, p.87, Remark (b)]).

Definition 5.2.1. Suppose that $\mathcal{L}$ is a prime perfect fgc Lie algebra. Then, the centroid $C(\mathcal{L})$ of $\mathcal{L}$ is an integral domain $[\mathrm{ABP}$, Lemma 3.3(i)]. Let $\overline{C(\mathcal{L})}$ denote an algebraic closure of the quotient field of $C(\mathcal{L})$, and put $\overline{\mathcal{L}}=\mathcal{L} \otimes_{C(\mathcal{L})} \overline{C(\mathcal{L})}$. Then, $\overline{\mathcal{L}}$ is a finite dimensional simple Lie algebra over $\overline{C(\mathcal{L})}[\mathrm{ABP}$, Prop. 8.7]. We define the absolute type of $\mathcal{L}$ to be the type of this algebra over $\overline{C(\mathcal{L})}$ (that is, the type of its root system). So the type of $\mathcal{L}$ is one of the reduced types $\mathrm{X}_{\ell}$ in the list (11).

Absolute type is an isomorphism invariant. That is, if $\mathcal{L}$ and $\mathcal{L}^{\prime}$ are prime perfect fgc Lie algebras and $\mathcal{L}$ is isomorphic to $\mathcal{L}^{\prime}$, then $\mathcal{L}$ and $\mathcal{L}^{\prime}$ have the same type $\mathrm{ABP}$, Proposition 8.15]. 
Proposition 5.2.2. Suppose that $\mathcal{L}$ is an fgc centreless Lie $\Lambda$-torus, where $\Lambda$ is a free abelian group of rank $n \geq 1$. Then $\mathcal{L}$ is prime and perfect and so its absolute type is defined. Moreover, if $\mathcal{L}$ is bi-isomorphic (or even just isomorphic as a Lie algebra) to a multiloop Lie torus $\operatorname{LT}(\mathfrak{s}, \boldsymbol{\sigma}, \mathfrak{h})$, then the absolute type of $\mathcal{L}$ is the type of the simple Lie algebra $\mathfrak{s}$ over $k$.

Proof. By the Realization Theorem, we can assume that $\mathcal{L}$ is equal to a multiloop Lie torus $\operatorname{LT}(\mathfrak{s}, \boldsymbol{\sigma}, \mathfrak{h})$. The result is now a special case of Theorem 8.16 of ABP] (which applies more generally to iterated loop algebras based on $\mathfrak{s}$ ).

5.3. The approach to classification. For the rest of the section, suppose that $\mathfrak{s}$ is a finite dimensional simple Lie algebra $\mathfrak{s}$ of type $X_{\ell}$. (We will use $\mathfrak{s}$ as the base algebra for our multiloop Lie tori.) Let

$$
A=\operatorname{Aut}_{k}(\mathfrak{s}) .
$$

Remark 5.3.1. Let $G$ be a finite abelian subgroup of $A$.

(i) Let $X(G)=\operatorname{Hom}\left(G, k^{\times}\right)$be the character group of $G$. Then we have the weight space decomposition

$$
\mathfrak{s}=\bigoplus_{\chi \in X(G)} \mathfrak{s}^{\chi}
$$

of $\mathfrak{s}$ relative to the action of $G$, where $\mathfrak{s}^{\chi}=\{x \in \mathfrak{s}: g(x)=\chi(g) x$ for $g \in G\}$. If $1 \in X(G)$ is the trivial character (that is, $1(\gamma)=1$ for all $\gamma \in \Gamma$ ), then $\mathfrak{s}^{1}=\mathfrak{s}^{G}$, the fixed point subalgebra of $G$, and each $\mathfrak{s}^{\chi}$ is an $\mathfrak{s}^{G}$-module under the adjoint action.

(ii) We will be interested in finite abelian subgroups $G$ of $A$ that satisfy the following three conditions:

(G1) $\mathfrak{g}:=\mathfrak{s}^{G}$ is a simple Lie algebra.

(G2) If $1 \neq \chi \in X(G)$ and $\mathfrak{s}^{\chi} \neq 0$, then $\mathfrak{s}^{\chi} \simeq U^{\chi} \oplus V^{\chi}$ as a $\mathfrak{g}$-module, where $\mathfrak{g}$ acts trivially on $U^{\chi}$ and either $V^{\chi}=0$ or $V^{\chi}$ satisfies condition (M).

$\left(\mathrm{G} 3_{n}\right) \operatorname{mg}(G) \leq n$.

(See Definition 3.2.3) It is clear that if $G$ satisfies (G1) (resp. (G1) and (G2); resp. $\left(\mathrm{G} 3_{n}\right)$ ), then any conjugate of $G$ in $A$ also satisfies (G1) (resp. (G1) and (G2); resp. $\left.\left(\mathrm{G} 3_{n}\right)\right)$.

(iii) Suppose that $\operatorname{mg}(G) \leq n$ and so $\operatorname{gs}_{n}(G) \neq \emptyset$. Then, $\mathrm{GL}_{n}(\mathbb{Z})$ acts on $\operatorname{gs}_{n}(G)$ on the right (see Remark (5.1.2)), and hence on the left by $P \cdot \boldsymbol{\sigma}=\boldsymbol{\sigma}^{P^{-1}}$. Also, the normalizer $N_{A}(G)$ of $G$ in $A$ acts on $\operatorname{gs}_{n}(G)$ on the left by conjugation:

$$
\rho \cdot \boldsymbol{\sigma}=\left(\rho \sigma_{1} \rho^{-1}, \ldots, \rho \sigma_{n} \rho^{-1}\right)
$$

for $\boldsymbol{\sigma}=\left(\sigma_{1}, \ldots, \sigma_{n}\right) \in \operatorname{gs}_{n}(G)$ and $\rho \in N_{A}(G)$. These left actions commute and so $N_{A}(G) \times \mathrm{GL}_{n}(\mathbb{Z})$ acts on the left on $\operatorname{gs}_{n}(G)$ by $(\rho, P) \cdot \boldsymbol{\sigma}=(\rho \cdot \boldsymbol{\sigma})^{P^{-1}}=\rho \cdot\left(\boldsymbol{\sigma}^{P^{-1}}\right)$.

We can now state our result on the classification of fgc centreless Lie tori up to bi-isomorphism.

Theorem 5.3.2. Suppose $n \geq 1$ and $\mathfrak{s}$ is a finite dimensional simple Lie algebra of type $X_{\ell}$. Set $A=\operatorname{Aut}_{k}(\mathfrak{s})$.

(i) Let $\left\{G_{i}\right\}_{i \in I}$ be a nonredundant set of representatives of the conjugacy classes of the finite abelian subgroups of $A$ that satisfy (G1), (G2) and $\left(G 3_{n}\right)$. 
(ii) For $i \in I$, let $\{\mathcal{O}(i, j)\}_{j \in J_{i}}$ be the set of orbits of the action of $N_{A}\left(G_{i}\right) \times$ $\mathrm{GL}_{n}(\mathbb{Z})$ on $\operatorname{gs}_{n}\left(G_{i}\right)$. (These orbits are unions of the orbits of $\mathrm{GL}_{n}(\mathbb{Z})$ on $\mathrm{gs}_{n}\left(G_{i}\right)$, and the latter orbits were calculated in Proposition 5.1.3.)

(iii) For $i \in I$ and $j \in J_{i}$, choose $\boldsymbol{\sigma}(i, j) \in \mathcal{O}(i, j)$ with the property that $\left|G_{i}\right|=$ $\left|\boldsymbol{\sigma}(i, j)_{1}\right| \ldots\left|\boldsymbol{\sigma}(i, j)_{n}\right|$. (This is possible by Proposition [5.1.3.)

(iv) For $i \in I$ and $j \in J_{i}$, let $\mathfrak{h}(i, j)$ be a Cartan subalgebra of $\mathfrak{s}^{\boldsymbol{\sigma}(i, j)}$.

Then, for $i \in I, j \in J_{i}, \boldsymbol{\sigma}(i, j)$ satisfies (A1)-(A3) (in Proposition 3.2.5) and $\mathrm{LT}(\mathfrak{s}, \boldsymbol{\sigma}(i, j), \mathfrak{h}(i, j))$ is a centreless Lie $\mathbb{Z}^{n}$-torus of absolute type $X_{\ell}$. Moreover, if $\Lambda$ is a free abelian group of rank $n$, then any fgc centreless Lie $\Lambda$-torus of absolute type $X_{\ell}$ is bi-isomorphic to exactly one of the Lie tori $\operatorname{LT}(\mathfrak{s}, \boldsymbol{\sigma}(i, j), \mathfrak{h}(i, j))$.

Proof. For the first statement, suppose that $i \in I$ and $j \in J_{i}$. Since $\boldsymbol{\sigma}(i, j) \in$ $\mathrm{gs}_{n}\left(G_{i}\right)$, we have

$$
G_{i}=\left\langle\sigma(i, j)_{1}, \ldots, \sigma(i, j)_{n}\right\rangle .
$$

Hence (A3) in Proposition 3.2.5 holds by the choice of $\boldsymbol{\sigma}(i, j)$ in (iii). Also, by (35), the grading of $\mathfrak{s}$ determined by $\boldsymbol{\sigma}(i, j)$ (as in Definition 3.1.2) has the same summands as the weight space decomposition of $\mathfrak{s}$ relative to the action of $G_{i}$. So, since $G_{i}$ satisfies (G1) and (G2), $\boldsymbol{\sigma}(i, j)$ satisfies (A1) and (A2). Thus, $\operatorname{LT}(\mathfrak{s}, \boldsymbol{\sigma}(i, j), \mathfrak{h}(i, j))$ is a centreless Lie $\mathbb{Z}^{n}$-torus by Proposition 3.2.5. Moreover, this Lie torus has absolute type $\mathrm{X}_{\ell}$ by Proposition 5.2 .2

For the second statement, suppose that $\mathcal{L}$ is an fgc centreless Lie $\Lambda$-torus of absolute type $\mathrm{X}_{\ell}$, where $\Lambda$ is free abelian of rank $n$. By Theorem 3.3.1 we can assume that $\mathcal{L}=\operatorname{LT}\left(\mathfrak{s}^{\prime}, \boldsymbol{\sigma}, \mathfrak{h}\right)$ is a multiloop Lie $\mathbb{Z}^{n}$-torus based on some finite dimensional simple Lie algebra $\mathfrak{s}^{\prime}$. Then, $\mathfrak{s}^{\prime}$ has absolute type $\mathrm{X}_{\ell}$ by Proposition 5.2.2, so we can assume that $\mathfrak{s}^{\prime}=\mathfrak{s}$. Next let $G=\left\langle\sigma_{1}, \ldots, \sigma_{n}\right\rangle$. Since $\boldsymbol{\sigma}$ satisfies (A1) and (A2), it follows as in the first part of the proof that $G$ satisfies (G1) and (G2). Also, clearly $G$ satisfies $\left(\mathrm{G} 3_{n}\right)$. Hence, $G$ is conjugate in $A$ to $G_{i}$ for some $i \in I$. So, by Theorem 4.1.1. we can assume that $G=G_{i}$ and thus $\boldsymbol{\sigma} \in \operatorname{gs}_{n}\left(G_{i}\right)$. Hence, $\boldsymbol{\sigma} \in \mathcal{O}(i, j)$ for some $j \in J_{i}$. So $\boldsymbol{\sigma}$ and $\boldsymbol{\sigma}(i, j)$ are both elements of the same orbit $\mathcal{O}(i, j)$ and hence, by Theorem 4.1.1, $\mathcal{L}(\mathfrak{s}, \boldsymbol{\sigma}, \mathfrak{h})$ is biisomorphic to $\operatorname{LT}(\mathfrak{s}, \boldsymbol{\sigma}(i, j), \mathfrak{h}(i, j))$.

For the uniqueness part of the second statement, suppose that the Lie $\mathbb{Z}^{n}$-tori $\operatorname{LT}(\mathfrak{s}, \boldsymbol{\sigma}(i, j), \mathfrak{h}(i, j))$ and $\operatorname{LT}\left(\mathfrak{s}, \boldsymbol{\sigma}\left(i^{\prime}, j^{\prime}\right), \mathfrak{h}\left(i^{\prime}, j^{\prime}\right)\right)$ are bi-isomorphic, where $i, i^{\prime} \in I$, $j \in J_{i}$ and $j^{\prime} \in J_{i^{\prime}}$. Then, by Corollary 4.1.4, $G_{i}$ is conjugate to $G_{i^{\prime}}$ in $A$, so $i^{\prime}=i$. Hence, again by Theorem 4.1.1, $\boldsymbol{\sigma}(i, j)$ and $\boldsymbol{\sigma}\left(i, j^{\prime}\right)$ are in the same orbit under the action of $N_{A}\left(G_{i}\right) \times \mathrm{GL}_{n}(\mathbb{Z})$ on $\mathrm{gs}_{n}\left(G_{i}\right)$, so $j=j^{\prime}$.

Remark 5.3.3. Suppose we have the assumptions and notation of Theorem 5.3.2, and let $i \in I$.

(i) We call the Lie tori $\operatorname{LT}(\mathfrak{s}, \boldsymbol{\sigma}(i, j), \mathfrak{h}(i, j)), j \in J_{i}$, the multiloop Lie tori corresponding to $G_{i}$.

(ii) If either $\operatorname{mg}\left(G_{i}\right)<n$ or the smallest invariant factor of $G_{i}$ is $\leq 4$, then it follows from Proposition 5.1 .3 that $N_{A}\left(G_{i}\right) \times \mathrm{GL}_{n}(\mathbb{Z})$ acts transitively on $\mathrm{gs}_{n}\left(G_{i}\right)$ and so there is only one multiloop Lie torus corresponding to $G_{i}$. This happens frequently in practice.

(iii) If $G_{i}$ is contained in a torus of $A$, then, by Corollary 4.1.6, we have $G_{i}=\{1\}$, so the only multiloop Lie torus corresponding to $G_{i}$ is the untwisted one. On the other hand, if $G_{i}$ is not contained in a torus of $A$, the multiloop Lie tori 
corresponding to $G_{i}$ are not isograded-isomorphic to the untwisted one (again by Corollary 4.1.6).

Example 5.3.4. As an example, we obtain the classification of fgc centreless Lie $\mathbb{Z}^{n}$-tori of absolute type $\mathrm{F}_{4}$, where $n \geq 1$. For this, let $\mathfrak{s}$ be the finite dimensional simple Lie algebra of type $\mathrm{F}_{4}$ and let $A=\operatorname{Aut}_{k}(\mathfrak{s})$. We use our results above and the work of C. Draper and C. Martin [DM], who found representatives of the conjugacy classes of finite abelian subgroups of $A$ that are not contained in a torus. They labeled these subgroups as I, II.1, II.2, II.3, II.4 and III.

Suppose that $G$ is a finite abelian subgroup of $A$ that satisfies (G1) and (G2), and suppose that $G$ is not contained in a torus. If $G$ has label I, II.2, II.3, II.4 or III, then $G$ does not satisfy both (G1) and (G2). So $G$ has label II.1. Then, $G=\left\langle\sigma_{1}, \sigma_{2}, \sigma_{3}\right\rangle \simeq \mathbb{Z}_{2}^{3}$, where, in the notation of [DM],

$$
\sigma_{1}=\widetilde{\sigma_{105}}, \quad \sigma_{2}=t_{-1,1,-1,1}^{\prime}, \quad \sigma_{3}=t_{1-, 1,-1,1}^{\prime} .
$$

Conversely, with this choice of $G, \mathfrak{s}^{G}$ is simple of type $\mathrm{A}_{1}$ and, for $1 \neq \chi \in X(G)$, $\mathfrak{s}^{\chi} \simeq U^{\chi} \oplus V^{\chi}$, where $U^{\chi}$ is 2-dimensional with trivial $\mathfrak{g}$-action and $V^{\chi}$ is the (5-dimensional) symmetric module for $\mathfrak{g}$. So (G1) and (G2) hold. Note also that $\operatorname{mg}(G)=3$ and the smallest invariant factor of $G$ is 2 .

So, by Theorem 5.3.2 and Remark 5.3.3, the fgc centreless Lie $\mathbb{Z}^{n}$-tori of absolute type $\mathrm{F}_{4}$ are the untwisted multiloop Lie torus and, if $n \geq 3$, the multiloop Lie torus $\operatorname{LT}(\mathfrak{s}, \boldsymbol{\sigma}, \mathfrak{h})$, where $\boldsymbol{\sigma}=\left(\sigma_{1}, \sigma_{2}, \sigma_{3}, 1, \ldots, 1\right)$ and $\mathfrak{h}$ is a Cartan subalgebra of $\mathfrak{s}^{\sigma}$.

As mentioned in the introduction, to obtain a classification up to isotopy of fgc centreless Lie $\mathbb{Z}^{n}$-tori of a given absolute type, one can, at least in principle, proceed as follows. First obtain a classification up to bi-isomorphism using the approach described in Theorem 5.3.2. Second, use Theorem 4.2.5 and Corollary 4.2.6 to determine which of the representatives of the bi-isomorphism classes are isotopic. This last step is easy for absolute type $\mathrm{F}_{4}$.

Example 5.3.5. We have carried out the classification of fgc centreless Lie $\mathbb{Z}^{n}$-tori of absolute type $\mathrm{F}_{4}$ up to bi-isomorphism in Example 5.3.4. The two representatives found there are not isotopic by Corollary 4.2.6. So there are precisely two fgc centreless Lie $\mathbb{Z}^{n}$-tori of absolute type $\mathrm{F}_{4}$ up to isotopy.

\section{ACKNOWLEDGEMENT}

The authors thank Christina Draper for a very helpful conversation related to her work with Candido Martin in $[\mathrm{DM}]$ on gradings of $F_{4}$.

\section{REFERENCES}

[AABGP] B. Allison, S. Azam, S. Berman, Y. Gao and A. Pianzola, Extended affine Lie algebras and their root systems, Mem. Amer. Math. Soc. 126 \#603 (1997). MR.1376741 (97i:17015)

[ABFP] B. Allison, S. Berman, J. Faulkner, and A. Pianzola, Realization of graded-simple algebras as loop algebras, Forum Mathematicum 20 (2008), 395-432. MR2418198

[ABGP] B. Allison, S. Berman, Y. Gao and A. Pianzola, A characterization of affine Kac-Moody Lie algebras, Comm. Math. Phys. 185 (1997), 671-688. MR1463057 (98h:17026)

[ABG] B. Allison, G. Benkart and Y. Gao, Lie algebras graded by the root system $B C_{r}, r \geq 2$, Mem. Amer. Math. Soc. 158 \#751 (2002). MR.1902499 (2003h:17038) 
[ABP] B.N. Allison, S. Berman and A. Pianzola, Iterated loop algebras, Pacific J. Math. 227 (2006), 1-42. MR2247871 (2007g:17022)

[AF] B. Allison and J. Faulkner, Isotopy for extended affine Lie algebras and Lie tori, arXiv:0709.1181v3 [math.RA] on arXiv.org.

[AG] B. Allison and Y. Gao, The root system and the core of an extended affine Lie algebra, Selecta Math. 7 (2001), 149-212. MR.1860013 (2002g:17041)

[BSZ] Y.A. Bahturin, I.P. Shestakov and M.V. Zaicev, Gradings on simple Jordan and Lie algebras, J. Algebra 283 (2005), 849-868. MR2111225 (2005i:17038)

[BN] G. Benkart and E. Neher, The centroid of extended affine and root graded Lie algebras, J. Pure Appl. Algebra 205 (2006), 117-145. MR2193194 (2006i:17043)

[BZ] G. Benkart and E. Zelmanov, Lie algebras graded by finite root systems and intersection matrix algebras, Invent. Math. 126 (1996), 1-45. MR.1408554 (97k:17044)

[BGK] S. Berman, Y. Gao and Y. Krylyuk, Quantum tori and the structure of elliptic quasisimple Lie algebras, J. Funct. Anal. 135 (1996), 339-389. MR.1370607 (97b:17007)

[BGKN] S. Berman, Y. Gao, Y. Krylyuk and E. Neher, The alternative torus and the structure of elliptic quasi-simple Lie algebras of type $A_{2}$, Trans. Amer. Math. Soc. 347 (1995), 4315-4363. MR:1303115 (96b:17009)

[BFM] A. Borel, R. Friedman and J.W. Morgan, Almost commuting elements in compact Lie groups, Mem. Amer. Math. Soc. 157 \#747 (2002). MR1895253(2003k:22006)

[B1] N. Bourbaki, Éléments de mathématique. Fasc. XXXIV. Groupes et algèbres de Lie. Chapitre IV: Groupes de Coxeter et systèmes de Tits. Chapitre V: Groupes engendrés par des réflexions. Chapitre VI: systèmes de racines, Actualités Scientifiques et Industrielles, No. 1337, Hermann, Paris, 1968, 288 pp. MR0240238 (39:1590)

[B2] N. Bourbaki, Éléments de mathématique. Fasc. XXXVIII: Groupes et algèbres de Lie. Chapitre VII: Sous-algèbres de Cartan, éléments réguliers. Chapitre VIII: Algèbres de Lie semi-simples déployées, Actualités Scientifiques et Industrielles, No. 1364. Hermann, Paris, 1975. 271 pp. MR0453824 (56:12077)

[DM] C. Draper and C. Martin, Gradings on the Albert Algebra and on $f_{4}$, arXiv:math/ 0703840v1 [math.RA] on arXiv.org.

[GP] P. Gille and A. Pianzola, Galois cohomology and forms of algebras over Laurent polynomial rings, Math. Ann. 338 (2007), 497-543. MR2302073 (2008b:20055)

[GOV] V.V. Gorbatsevich, A.L. Onishchik and E.B. Vinberg, Structure of Lie groups and Lie algebras, in Lie Groups and Lie Algebras III, Encyclopaedia of Mathematical Sciences Vol. 41, A.L. Onishchik and E.B. Vinberg, editors, Springer-Verlag, Berlin, 1994. MR.1349140 (96d:22001)

[H] J. Humphreys, Linear algebraic groups, Springer-Verlag, New York, 1975. MR0396773 $(53: 633)$

[J] N. Jacobson, Lie algebras, Dover, New York, 1979. MR559927(80k:17001)

[K] V.G. Kac, Infinite dimensional Lie algebras, Third edition, Cambridge University Press, Cambridge, 1990. MR:1104219 (92k:17038)

[KS] V.G. Kac and A.V. Smilga, Vacuum structure in supersymmetric Yang-Mills theories with any gauge group. The many faces of the superworld, 185-234, World Sci. Publ., River Edge, NJ, 2000. MR1885976 (2003k:22031)

[KW] V.G. Kac and S.P. Wang, On automorphisms of Kac-Moody algebras and groups, Advances in Math. 92 (1992), 129-195. MR.1155464 (93f:17041)

[Mc] K. McCrimmon, A taste of Jordan algebras, Springer, New York, 2004. MR2014924 (2004i:17001)

[N1] E. Neher, Lie tori, C.R. Math. Acad. Sci. Soc. R. Can., 26 (2004), no. 3, pp. 84-89. MR2083841 (2005d:17030)

[N2] E. Neher, Extended affine Lie algebras, C.R. Math. Acad. Sci. Soc. R. Can., 26 (2004), no. 3, pp. 90-96. MR2083842(2005f:17024)

[P] A. Pianzola, On automorphisms of semisimple Lie algebras, Algebras Groups Geom. 2 (1985), 95-116. MR901575 (88h:17013)

[Sel] G.B. Seligman, Rational methods in Lie algebras, Lect. Notes in Pure and Applied Math. 27, Marcel Dekker, New York, 1976. MR0427394 (55:428)

[vdL] J. van de Leur, Twisted toroidal Lie algebras, preprint, arXiv math/0106119 v1, 2001.

[Y1] Y. Yoshii, Coordinate algebras of extended affine Lie algebras of type $A_{1}$, J. Algebra 234 (2000), pp. 128-168. MR.1799481 (2001i:17031) 
[Y2] Y. Yoshii, Lie tori-A simple characterization of extended affine Lie algebras, Publ. Res. Inst. Math. Sci. 42 (2006), 739-762. MR2266995 (2007h:17027)

[Y3] Y. Yoshii, Root systems extended by an abelian group and their Lie algebras, J. Lie Theory 14 (2004), pp. 371-374. MR.2066861 (2005e:17016)

Department of Mathematics and Statistics, University of Victoria, PO Box 3060 STN CSC, Victoria, British Columbia, Canada V8W 3R4

E-mail address: ballison@uvic.ca

Saskatoon, Saskatchewan, Canada

E-mail address: sberman@shaw.ca

Department of Mathematics, University of Virginia, Kerchof Hall, P.O. Box 400137 , Charlottesville, Virginia 22904-4137

E-mail address: jrf@virginia.edu

Department of Mathematical and Statistical Sciences, University of Alberta, Edmonton, Alberta, Canada T6G 2G1

E-mail address: a.pianzola@ualberta.ca 